Pontifícia Universidade $C_{\text {atólica }}$

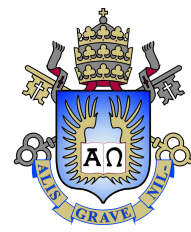

Rafael Segadas dos Santos

Cáusticas de Wigner e Conjuntos de Medida de Largura Constante em Planos Normados com Bolas Unitárias Suaves ou Poligonais

Tese de Doutorado

Tese apresentada como requisito parcial para obtenção do grau de Doutor pelo Programa de Pós-graduação em Matemática da PUC-Rio.

Orientador: Prof. Marcos Craizer 

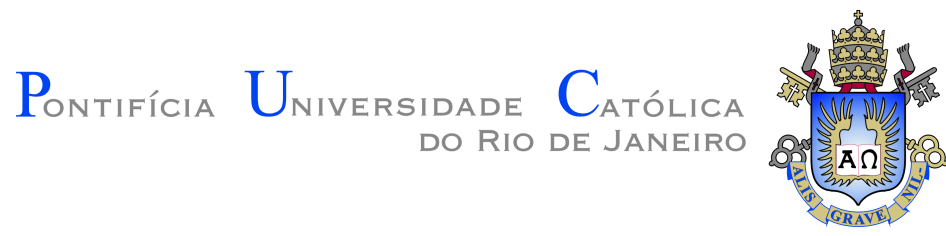

Rafael Segadas dos Santos

\title{
Cáusticas de Wigner e Conjuntos de Medida de Largura Constante em Planos Normados com Bolas Unitárias Suaves ou Poligonais
}

Tese apresentada como requisito parcial para obtenção do grau de Doutor pelo Programa de Pós-graduação em Matemática da PUC-Rio. Aprovada pela Comissão Examinadora abaixo.

\author{
Prof. Marcos Craizer \\ Orientador \\ Departamento de Matemática - PUC-Rio \\ Prof. Ralph Costa Teixeira \\ Departamento de Matemática - UFF \\ Prof. Carlos Hugo Jimenez \\ Departamento de Matemática - PUC-Rio
}

Prof. Silvius Klein

Departamento de Matemática - PUC-Rio

Prof. Vitor Balestro

Departamento de Matemática - UFF

Prof. Moacyr Alvim Horta Barbosa da Silva

Departamento de Matemática - Emap-FGV

Rio de Janeiro, 20 de Setembro de 2019 
Todos os direitos reservados. É proibida a reprodução total ou parcial do trabalho sem autorização da universidade, do autor e do orientador.

Rafael Segadas dos Santos

Ficha Catalográfica

Santos, Rafael Segadas

Cáusticas de Wigner e Conjuntos de Medida de Largura Constante em Planos Normados com Bolas Unitárias Suaves ou Poligonais / Rafael Segadas dos Santos; orientador: Marcos Craizer. - Rio de janeiro: PUC-Rio, Departamento de Matemática, 2019.

v., 94 f: il. color. ; $30 \mathrm{~cm}$

Tese (doutorado) - Pontifícia Universidade Católica do Rio de Janeiro, Departamento de Matemática.

Inclui bibliografia

1. Matemática - Teses. 2. Plano de Minkowski;. 3. Cáusticas de Wigner;. 4. Curvas de Largura Constante;. 5. Equações de Sturm-Liouville;. 6. Desigualdades Isoperimétricas;. I. Craizer, Marcos. II. Pontifícia Universidade Católica do Rio de Janeiro. Departamento de Matemática. III. Título. 


\section{Agradecimentos}

Primeiramente a Deus por seu amor incondicional, sua direção e força para não desistir.

O amor da minha vida Priscila Carrati, que sempre me apoia e que sem seu suporte esse projeto não seria possível.

Minhas filhas Maria Rafaela e Angelina que me motivam a caminhar e que são minha alegria.

Meus Pais e minha irmã Gisele pelo apoio e orações.

Meu Orientador Marcos Craizer que tanto me ajudou e acreditou em mim.

A PUC, funcionários e professores.

A banca de defesa, pela leitura da tese e sugestões.

Aos meus colegas de doutorado, pelo apoio.

O presente trabalho foi realizado com apoio da Coordenação de Aperfeiçoamento de Pessoal de Nível Superior - Brasil (CAPES) - Código de Financiamento 001. 


\section{Resumo}

Santos, Rafael Segadas; Craizer, Marcos. Cáusticas de Wigner e Conjuntos de Medida de Largura Constante em Planos Normados com Bolas Unitárias Suaves ou Poligonais. Rio de Janeiro, 2019. 94p. Tese de Doutorado - Departamento de Matemática, Pontifícia Universidade Católica do Rio de Janeiro.

Em [23] são apresentadas igualdades e desigualdades isoperimétricas relacionadas à Cáustica de Wigner $(C W)$ e ao Conjunto de Medida de Largura Constante $(C M L C)$. Neste trabalho nós estendemos estes resultados para planos normados com bolas unitárias quadraticamente convexas ou bolas unitárias poligonais. Estes conjuntos $C W$ e $C M L C$ estão fortemente relacionados às ciclóides, que são curvas cujas funções suporte generalizam a base de Fourier ( [6], [7]). Uma característica importante deste trabalho é a analogia direta entre os casos contínuo e discreto.

\section{Palavras-chave}

Plano de Minkowski; Cáusticas de Wigner; Curvas de Largura Constante; Equações de Sturm-Liouville; Desigualdades Isoperimétricas; 


\section{Abstract}

Santos, Rafael Segadas; Craizer, Marcos (Advisor). Wigner Caustics and Constant Width Measure Sets in Normed Planes with Smooth or Polygonal Unit Balls. Rio de Janeiro, 2019. 94p. Tese de doutorado - Departamento de Matemática, Pontifícia Universidade Católica do Rio de Janeiro.

In [23], some isoperimetric equalities and inequalities related to the Wigner Caustic $(W C)$ and the Constant Width Measure Set $(C W M S)$ are proved. In this work, we generalize these results to normed planes with quadratically convex unitary ball or polygonal unitary balls. The $W C$ and $C W M S$ are closely related to cycloids, which are curves whose support functions generalize the Fourier basis ( [6], [7]). An important aspect of our work is the direct analogy between the smooth and discrete cases.

\section{Keywords}

Minkowski Planes; Wigner Caustics; Constant Width Curves; Sturm-Liouville Equations; Isoperimetric Inequalities; 


\section{Sumário}

1 Introdução $\quad 10$

2 Resultados Preliminares $\quad 14$

2.1 Plano Normado 14

$\begin{array}{lll}2.2 & \text { Exemplos } & 15\end{array}$

2.3 Curvas Legendrianas 16

2.4 Círculo Unitário Dual, Função Suporte e raio de Curvatura 18

2.5 Exemplo 20

3 Cáustica de Wigner e Conjunto de Medida de Largura Constante 21

3.1 Cáustica De Wigner e Curvas de Largura Constante 22

3.2 Conjunto de Medida de Largura Constante e Curvas Simétricas 25

3.3 Decomposição da curva $\gamma$ como soma de Caústica de Wigner, CMLC e bola unitária. $\quad 28$

3.4 Cuspides CMLC e Cáustica de Wigner 28

3.5 Áreas com sinal de CW e CMLC 29

$\begin{array}{lll}3.6 & \text { Evolutas } & 30\end{array}$

3.7 Exemplos 32

4 Igualdade Isoperimétrica em Plano Normado 35

5 Ciclóides e Base Ortonormal $\quad 38$

5.1 Áreas e Comprimentos $\quad 42$

5.2 Outra prova - Igualdade Isoperimétrica em Plano Normado 45

6 Circulo unitário e dual discretos, Função Suporte e Raio de Curvatura 47

6.1 Exemplos 49

$7 \quad$ Cáustica de Wigner e Conjunto de Medida de Largura Constante-Discreto 51

7.1 Cáustica de Wigner e Curvas de Largura Constante 52

7.2 Conjunto de Medida de Largura Constante e Curvas Simétricas 55

7.3 Decomposição do polígono $\mathrm{P}$ como soma de Caústica de Wigner, CMLC e bola unitária. $\quad 58$

7.4 Cúspides CMLC e Cáustica de Wigner 58

7.5 Áreas com sinal de CW e CMLC 59

$\begin{array}{lll}7.6 & \text { Evolutas } & 60\end{array}$

$\begin{array}{lll}7.7 & \text { Exemplos } & 63\end{array}$

8 Igualdade Isoperimétrica em Plano Normado - Discreto 65

8.1 Exemplo 68

9 Ciclóides e Base Ortonormal - Discreto 69

9.1 Áreas e Comprimentos $\quad 72$

9.2 Outra Prova - Igualdade Isoperimétrica em Plano Normado - Discreto 76 
10 Curvas Rosáceas $\quad 78$

10.1 Exemplo 78

10.2 Cáustica de Wigner 79

10.3 Conjunto de Medida de Largura Constante 80

10.4 Decomposição da curva $\gamma \in \mathscr{H}_{m}$ como soma de Caústica de Wigner, CMLC e bola unitária. $\quad 80$

10.5 Cuspides CMLC e Cáustica de Wigner 81

10.6 Exemplo 82

10.7 Igualdade Isoperimétrica em Plano Normado para $m$-rosáceas 83

11 Curvas Rosáceas - Discretas $\quad 85$

$\begin{array}{ll}11.1 \text { Cáustica de Wigner } & 86\end{array}$

11.2 Conjunto de Medida de Largura Constante 86

11.3 Decomposição do polígono $P \in C_{U}-m$ como soma de Caústica de Wigner, CMLC e bola unitária.

11.4 Cúspides CMLC e Cáustica de Wigner 87

11.5 Exemplo 88

11.6 Igualdade Isoperimétrica em Plano Normado para $m$-rosáceas discretas 89

$\begin{array}{ll}11.7 \text { Trabalhos Futuros } & 91\end{array}$

$\begin{array}{ll}\text { Referências bibliográficas } & 93\end{array}$ 


\section{Lista de figuras}

$\begin{array}{lll}\text { Figura 1.1 Tangentes paralelas em } \gamma . & 11\end{array}$

Figura 2.1 Bola unitária $U=\left\{(x, y) \in \mathbb{R}^{2} ;|x|^{3}+|y|^{3} \leq 1\right\} \quad 15$

$\begin{array}{lll}\text { Figura 2.2 } & \text { Bola unitária } U \text { como hexágono regular } & 16\end{array}$

Figura 2.3 Curva $\gamma(t)=\left(t^{2}, t^{3}\right)$ com cúspide 3/2 na origem. $\quad 17$

Figura 2.4 Curva $\gamma(t)$ com cúspide 5/4 em $(2,0)$ e Taylor. $\quad 17$

Figura 2.5 Interpretação geométrica da $U$-função suporte $\quad 19$

$\begin{array}{lll}\text { Figura 2.6 } & \text { Bola unitária } U \text { e seu dual } V & 20\end{array}$

Figura 3.1 Interpretação geométrica de curva $\gamma$ com largura constante. 22

Figura 3.2 Curva $\gamma$ de largura constante, $C M L C(\gamma)$ e $C W(\gamma) \quad 33$

Figura 3.3 Curva $\gamma$ simétrica, Cáustica $C W(\gamma)$ e $C M L C(\gamma) \quad 34$

Figura 3.4 Curva $\gamma$, Cáustica $C W(\gamma)$ e $C M L C(\gamma)$

Figura 6.1 Bola unitária $U$ e seu dual $V \quad 49$

Figura 6.2 Polígono $P \in C_{U}$ onde $r=\left(\frac{1}{2}, 1, \frac{1}{2}, \frac{3}{4}, \frac{3}{4}, \frac{3}{4}\right)$ e bola unitária 50

Figura 7.1 Polígono P, Cáustica de Wigner e CMLC C 64

$\begin{array}{llr}\text { Figura } 10.1 & 79\end{array}$

Figura 10.2 2-rosácea, CW, CMLC 82

Figura 11.1 Bola unitária $U$ e 2-rosácea $P \quad 85$

Figura 11.2 Bola U, 2-rosácea P, CW, CMLC 89 


\section{Introdução}

A Desigualdade Isoperimétrica clássica em um plano normado, também chamado plano de Minkowski, diz o seguinte:

Teorema 1.1 (Desigualdade Isoperimétrica). Seja $\gamma$ uma curva fechada simples em um plano normado. Então

$$
L_{v}^{2}(\gamma) \geq 4 A_{U} A_{\gamma}
$$

onde $A_{U}$ e $A_{\gamma}$ indicam respectivamente a área da bola unitária e a área da região limitada por $\gamma$ e $L_{v}(\gamma)$ indica o comprimento dual de $\gamma$. A igualdade ocorre se, e só se, $\gamma$ é múltiplo da bola unitária.

Um dos principais problemas em geometria plana é determinar quais curvas têm área máxima para um perímetro dado. Uma das primeiras referências ao problema isoperimétrico, que vem sendo estudado desde a antiguidade, é feita no poema latino Eneida, obra de Virgílio, onde se descreve a história de Dido, rainha de Cartago. Concluímos que, possivelmente, se conhecia a solução do problema isoperimétrico devido a solução em formato de círculo apresentada por Dido. A seguir está uma versão dessa história, conhecida como "A Lenda de Dido".

“...Dido consegue fugir com alguns amigos e partidários, levando consigo as riquezas do marido. Chegando a Costa do Mediterrâneo, norte da África, Dido resolve ficar e formar sua nova pátria. Ela negocia com o Rei Jarbas a compra de terras e ficou acertado que poderia comprar apenas a quantidade de terra que conseguisse cercar usando a pele de 1 (um) único touro. O pedido é aceito e Dido logo manda cortar o couro de um touro em estreitas tiras com o qual cercou uma imensa área de forma circular onde construiu a cidade com o nome de Birsa (couro). Em torno dessa cidade começa a se formar outra, Cartago, que logo se torna próspera." Retirado de [1]. 
Uma das primeiras demonstrações da Desigualdade Isoperimétrica foi obtida por Steiner [20], no século 19. Após, houve muitas outras provas e aplicações desse teorema; por exemplo, [2], [10], [12], [13], [14], [17], [19], [20], [21], [23], [24]. Em 1902, Hurwitz [13], apresentou uma prova para caso euclidiano utilizando análise de Fourier, já para no caso não euclidiano, podemos encontrar uma prova da Desigualdade Isoperimétrica em [21]. Mais recentemente Zwierzyński apresentou em [23], [24], para o caso euclidiano, versões "aprimoradas" da Desigualdade Isoperimétrica, envolvendo a Cáustica de Wigner $(C W)$ e o Conjunto de Medida de Largura Constante $(C M L C)$ ver teoremas (1.2) e (1.3). A Cáustica de Wigner pode ser definida como o lugar geométrico dos pontos médios dos pontos de uma curva $\gamma$ onde suas tangentes são paralelas, ver figura (1.1). Para mais detalhes sobre Cáustica de Wigner ver [4] e [11]. Já o $C M L C$ é definido, a menos de uma translação, como o conjunto das semi-cordas em pontos de $\gamma$ onde suas tangentes são paralelas.

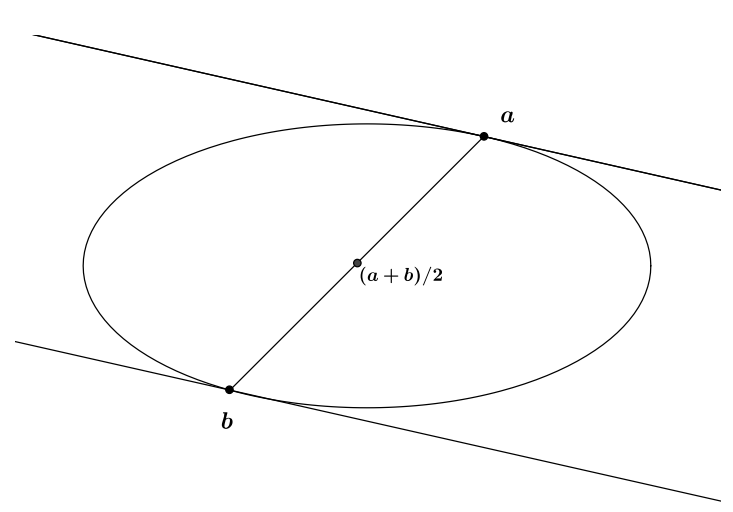

Figura 1.1: Tangentes paralelas em $\gamma$.

Teorema 1.2 (Zwierzyński). Seja $\gamma$ uma oval. Então

$$
L_{\gamma}^{2}(\gamma) \geq 4 \pi A_{\gamma}+8 \pi\left|\tilde{A}_{C W}\right|
$$

onde $\tilde{A}_{C W}$ indica a área com sinal da Cáustica de Wigner, $L_{v}(\gamma)$ e $A_{\gamma}$ indicam respectivamente o comprimento euclidiano e e área de $\gamma$. A igualdade ocorre se, e só se $\gamma$ é múltiplo da bola unitária. 
Teorema 1.3 (Zwierzyński). Seja $\gamma$ uma oval. Então

$$
L_{\gamma}^{2}(\gamma)=4 \pi A_{\gamma}+8 \pi\left|\tilde{A}_{C W}\right|+\pi\left|\tilde{A}_{C M L C}\right|
$$

onde $\tilde{A}_{C W}$ e $\tilde{A}_{C M L C}$ indicam respectivamente a área com sinal da Cáustica de Wigner e do Conjunto de Medida de Largura Constante, $L_{v}(\gamma)$ e $A_{\gamma}$ indicam respectivamente o comprimento euclidiano e e área de $\gamma$.

Motivados pelos teoremas (1.2) e (1.3) (ver [23], [24]), que são um refinamento da equação (1.1), definimos o $C M L C$ em um plano normado não necessariamente Euclidiano. O estudo deste conjunto levou a uma igualdade isoperimétrica análoga a de [23], porém desta vez em uma norma arbitrária com bola unitária quadraticamente convexa ou poligonal. No caso contínuo trabalhamos com espaço de curvas não necessariamente convexas, parametrizadas pelo ângulo que a reta tangente faz com uma direção fixa. Essas curvas são chamadas de "porco-espinho", "hedghog"em inglês, "hérissons"em francês. Já no caso discreto utilizamos como espaço de "curvas" polígonos fechados cujos lados são paralelos ao da bola unitária. Para métrica de Minkowski utilizamos os artigos de [6] e [7] e fazemos uma conexão entre ciclóides, Cáustica de Wigner e $C M L C$.

Dado um plano normado $U$, chamamos de $U$-ciclóides as curvas planas que são homotéticas a sua dupla $U$-evoluta. Acontece que o raio de curvatura e a função de suporte da $U$-ciclóide satisfazem uma equação diferencial do tipo Sturm - Liouville. Ao estudar essa equação podemos encontrar uma base ortonormal de $C^{0}\left(S^{1}\right)$ com uma decomposição natural em funções simétricas e anti-simétricas. Provamos nos teoremas (5.8) e (9.6), que a base ortonormal é na verdade o conjunto de funções suporte de Cáusticas de Wigner e Conjunto de Medida de Largura Constante. De modo análogo definimos ciclóides e evolutas discretas.

Em termos de função suporte, a Cáustica de Wigner e o Conjunto de Medida de Largura Constante podem ser considerados como a projeção nos espaços das funções ímpares e pares, respectivamente no caso de curvas com período $2 \pi$. Por outro lado, a série de Fourier é constituída de funções pares e ímpares. Sendo assim, em termos desta base, as operações $C W$ e $C M L C$ correspondem simplesmente a zerar os coeficientes dos termos pares e ímpares, respectivamente. Essa representação se mantém verdadeira em planos normados com bolas unitárias suaves estritamente convexas e também para 
bolas unitárias poligonais. Para normas não-euclidianas, devemos considerar as bases ortonormais construídas em [6] e [7].

Em [25], as noções de $C M L C$ e $C W$ são estendidas para $m$-rosáceas, que são curvas parametrizadas pelo ângulo que a reta tangente faz com uma direção fixa e dão $m$ voltas. Nesse trabalho estendemos as definições de $C W$ e $C M L C$ para $m$-rosáceas em um plano normado e provamos a correspondente Igualdade Isoperimétrica. Esta igualdade vale tanto para planos normados com bola unitária quadraticamente convexa como com bola poligonal.

No capítulo 2 e 6 apresentamos resultados preliminares. Nos capítulos 3 (contínuo) e 7 (discreto) apresentamos as definições e propriedades das Cáusticas de Wigner e Conjuntos de Medida de Largura Constante para curvas $2 \pi$-periódicas e a decomposição dessa curva em função desses conjuntos. Nos capítulos 4 (contínuo) e 8 (discreto) demonstramos a Igualdade Isoperimétrica em Plano Normado para curvas $2 \pi$-periódicas. Nos capítulos 5 (contínuo) e 9(discreto) apresentamos as ciclóides e bases ortonormais e uma outra prova para a Igualdade Isoperimétrica em Planos Normados para curvas $2 \pi$-periódicas. No capítulo 10(contínuo) e 11(discreto) apresentamos curvas rosáceas e Igualdade Isoperimétrica em Planos Normados para $m$-rosáceas.

\section{Notação}

Seja $v, w \in \mathbb{R}^{2}$ vetores. Nós denotamos o determinante cujas colunas são as coordenadas de $v$ e $w$ por $[v, w]$. 


\section{Resultados Preliminares}

\section{1}

\section{Plano Normado}

Chamamos de plano normado ou plano de Minkowski o plano $\mathbb{R}^{2}$ munido com uma norma qualquer \|.\|. Essa norma é chamada de norma de Minkowski.

A partir da norma $\|$.$\| podemos provar que o conjunto U=\{x \in$ $\mathbb{R}^{2},\|x\| \leq 1$ \} é convexo e simétrico. Reciprocamente se um conjunto $U$ do plano é convexo e simétrico ele define uma norma $\|\cdot\|_{U}$, chamada de norma induzida por $U$. A definição da norma é como segue: para todo $x \in \mathbb{R}^{2}$ podemos escrever $x=t u$, onde $t \geq 0$ e $u$ está na fronteira de $U$, então $\|x\|_{U}=t$.

\section{Lema 2.1.}

1. Se um conjunto $U$ é convexo e simétrico então $\|.\|_{U}$ é uma norma.

2. Se $\|$.$\| é uma norma em \mathbb{R}^{2}$ então o conjunto $\left\{x \in \mathbb{R}^{2},\|x\| \leq 1\right\}$ é convexo e simétrico.

Demonstração. (1)(Desigualdade Triangular) Sejam $x, y \in \mathbb{R}^{2}$ não nulos e $\alpha \in[0,1]$. Então $\frac{x}{\|x\|_{U}} \mathrm{e} \frac{y}{\|y\|_{U}}$ pertencem à fronteira de $U$. Como $U$ é convexa

$$
\alpha \frac{x}{\|x\|_{U}}+(1-\alpha) \frac{y}{\|y\|_{U}} \in U
$$

Se tomarmos $\alpha=\frac{\|x\|_{U}}{\|x\|_{U}+\|y\|_{U}}$ teremos $\left\|\frac{x}{\|x\|_{U}+\|y\|_{U}}+\frac{y}{\|x\|_{U}+\|y\|_{U}}\right\|_{U} \leq 1$

que implica $\|x+y\|_{U} \leq\|x\|_{U}+\|y\|_{U}$.

(2) A simetria vem da própria definição de $U$. Sejam $x, y \in U$ e $\alpha \in[0,1]$. Então por hipótese

$$
\|\alpha x+(1-\alpha) y\|_{U} \leq \alpha\|x\|_{U}+(1-\alpha)\|y\|_{U} \leq \alpha+(1-\alpha)=1
$$

ou seja, $\alpha x+(1-\alpha) y \in U$. Portanto $U$ é convexa. 
Para mais detalhes ver [21, p.17]. Vale destacar que a norma induzida por $U$ também pode ser definida por $\|x\|_{U}=\inf \{\lambda>0: x \in \lambda U\}$, chamada de Funcional de Minkowski ou Função "Gauge".

\section{2}

\section{Exemplos}

Exemplo 2.2. Considere como bola unitária $U=\left\{(x, y) \in \mathbb{R}^{2} ;|x|^{3}+|y|^{3} \leq 1\right\}$ cuja fronteira pode ser parametrizada por $u(t)=\left((\cos t)^{2 / 3},(\sin t)^{2 / 3}\right), 0 \leq t \leq$ $2 \pi$.

Se $A=\left(\frac{3}{2}\left(\frac{1}{4}\right)^{\frac{1}{3}}, \frac{3}{2}\left(\frac{3}{4}\right)^{\frac{1}{3}}\right)$ então $\|A\|_{U}=\frac{3}{2}$, onde $u_{A}=\left(\left(\frac{1}{4}\right)^{\frac{1}{3}},\left(\frac{3}{4}\right)^{\frac{1}{3}}\right)$, $\left\|u_{A}\right\|_{U}=1$ e $A=\frac{3}{2} u_{A}$.

Se $B=\left(-(4)^{\frac{1}{3}},-(4)^{\frac{1}{3}}\right)$ então $\|B\|_{U}=2$, onde $u_{B}=\left(-\left(\frac{1}{2}\right)^{\frac{1}{3}},-\left(\frac{1}{2}\right)^{\frac{1}{3}}\right)$, $\left\|u_{B}\right\|_{U}=1$ e $B=2 u_{B}$.

Se $C=\left(\left(\frac{3}{32}\right)^{\frac{1}{3}},-\left(\frac{1}{32}\right)^{\frac{1}{3}}\right)$ então $\|C\|_{U}=\frac{1}{2}$, onde $u_{C}=\left(\left(\frac{3}{4}\right)^{\frac{1}{3}},-\left(\frac{1}{4}\right)^{\frac{1}{3}}\right)$, $\left\|u_{C}\right\|_{U}=1$ e $C=\frac{1}{2} u_{C}$.Ver figura 2.1

Exemplo 2.3. Considere como bola unitária $U$ um hexágono (com interior) regular centrado na origem com um de seus vértices sobre o eixo y. Vale destacar que nesse caso o perímetro da bola é 6 (Teorema de Golab, [21]).

Considere os pontos $A, B$, e $C$, onde $A=2 u_{A}, B=\frac{3}{2} u_{B}, C=\frac{1}{2} u_{C}$ e $u_{A}, u_{B}, u_{C}$ estão sobre o hexágono. Nesse caso teremos $\|A\|_{U}=2,\|B\|_{U}=\frac{3}{2},\|C\|_{U}=\frac{1}{2}$. Ver figura 2.2

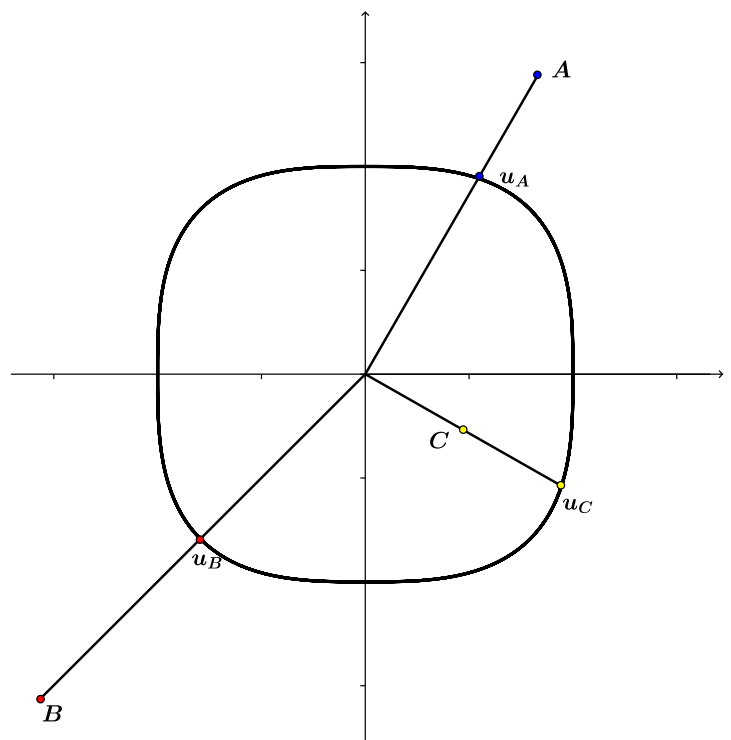

Figura 2.1: Bola unitária $U=\left\{(x, y) \in \mathbb{R}^{2} ;|x|^{3}+|y|^{3} \leq 1\right\}$ 


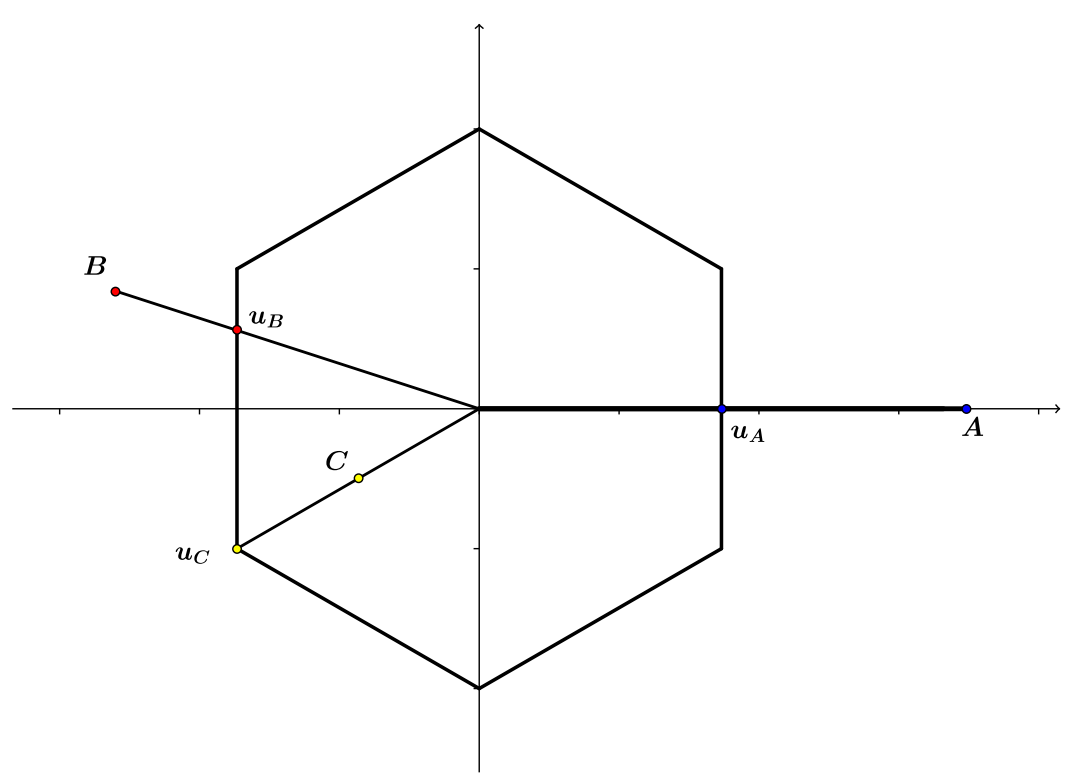

Figura 2.2: Bola unitária $U$ como hexágono regular

\section{3}

\section{Curvas Legendrianas}

Definição 2.4. Seja $I \subset \mathbb{R}$ um intervalo. Uma curva suave $\gamma: I \rightarrow R^{2} e ́$ chamada de Legendriana, se existe um mapa suave $\nu: I \rightarrow S^{1}$ tal que para todo $t \in I$ :

- $\left[\nu(t), \gamma^{\prime}(t)\right]=0$

- se $\gamma^{\prime}(t)=0$ então $\nu^{\prime}(t) \neq 0$.

Exemplo 2.5. Um típico exemplo de curva Legendriana é $\gamma: I \rightarrow R^{2}$, onde $\gamma^{\prime}(t) \neq 0, \forall t \in I$. Nesse caso basta considerar $\nu: I \rightarrow S^{1}, \nu(t)=\frac{\gamma^{\prime}(t)}{\left\|\gamma^{\prime}(t)\right\|}$.

Considere agora $\gamma: I \rightarrow R^{2}$ e $\nu: I \rightarrow S^{1}$ tal que $\gamma(t)=\left(t^{m}, t^{n}\right)$, $m, n$ inteiros, $1<m<n$ e $\nu(t)=\frac{1}{\sqrt{m^{2}+n^{2} t^{2(n-m)}}}\left(m, n t^{n-m}\right)$. Observe que se $\nu^{\prime}(0) \neq 0$ então $\gamma$ é uma curva Legendriana. Por outro lado $\nu^{\prime}(0) \neq 0$ ocorre se, e somente se, $n=m+1$. Em particular se $\gamma^{\prime \prime}(0) \neq 0$ teremos $m=2 \mathrm{e}$ $n=3$, isto é $\gamma(t)=\left(t^{2}, t^{3}\right)$, ver figura 2.3. Concluímos que todos os cúspides das curvas Legendrianas são da forma $(n+1) / n$ e se $\gamma$ não possuir pontos de inflexão então seus cúspides são da forma $3 / 2$. Para mais detalhes sobre curvas Legendrianas ver [9] e [6]. 
Exemplo 2.6. Considere a curva $\gamma(t)=(3 \cos 3 t-\cos 5 t)(\cos t, \sin t)+$ $(-9 \sin 3 t+5 \sin 5 t)(-\sin t, \cos t)$. Observe que $\gamma^{\prime}(0)=0$ e $\gamma^{\prime \prime}(0)=0$. Utilizando polinômio de Taylor em $t=0$ vemos que essa singularidade é do tipo 5/4. Ver figura 2.4.

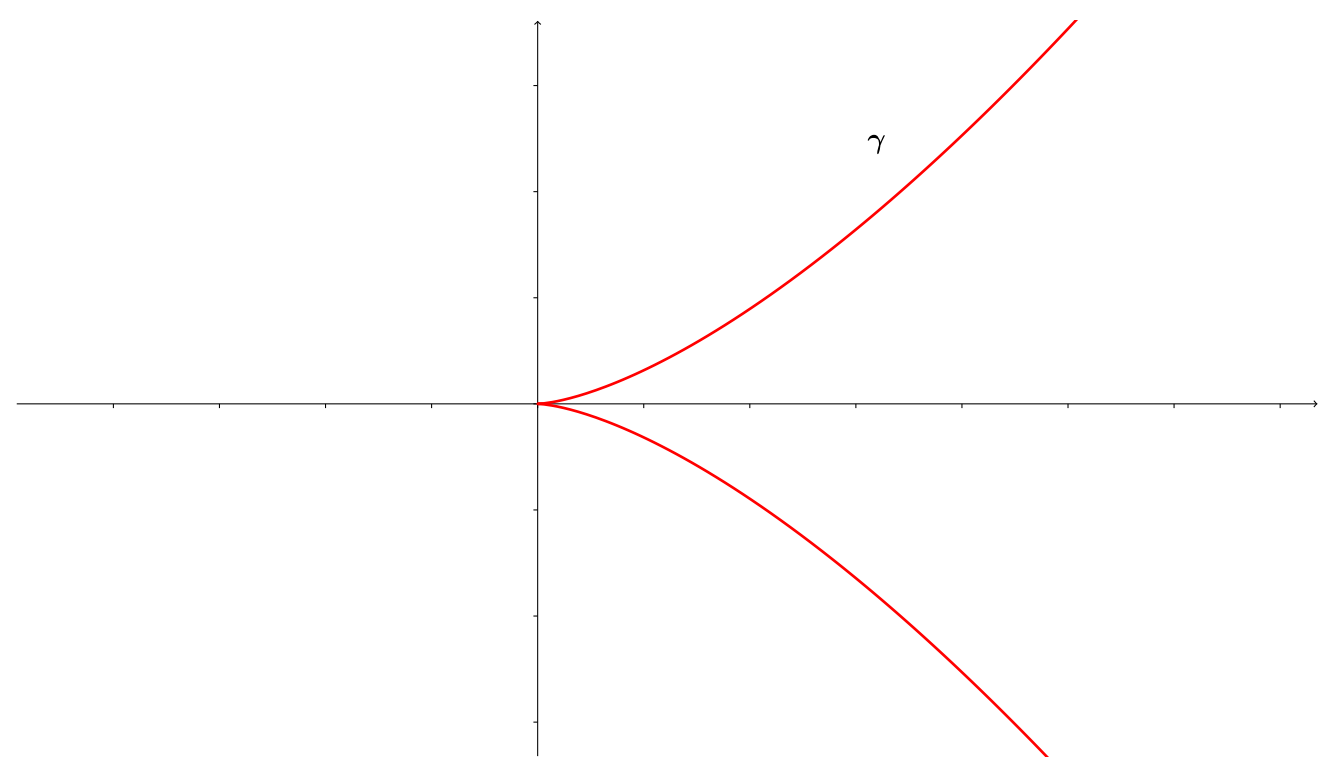

Figura 2.3: Curva $\gamma(t)=\left(t^{2}, t^{3}\right)$ com cúspide $3 / 2$ na origem.

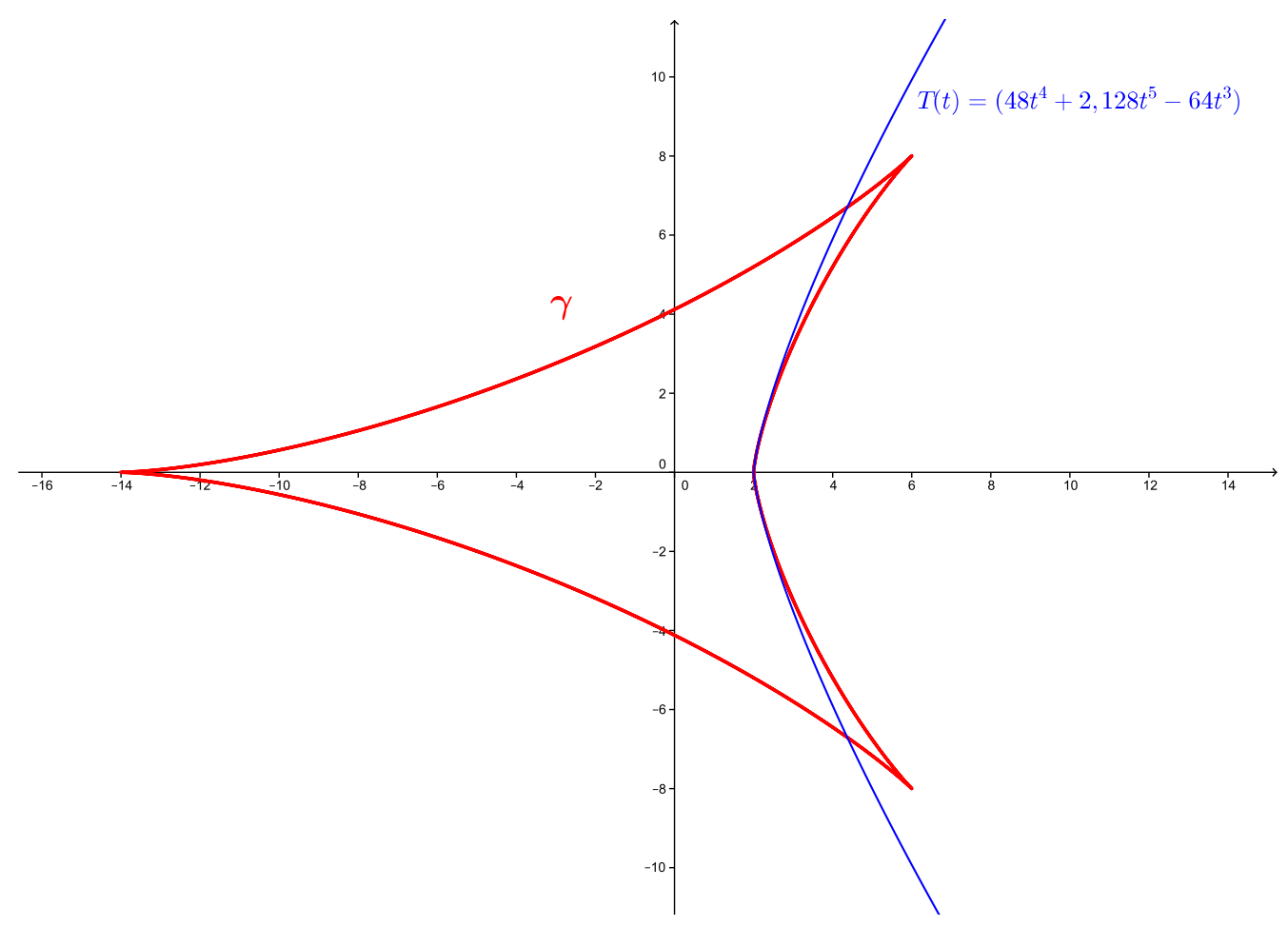

Figura 2.4: Curva $\gamma(t)$ com cúspide $5 / 4$ em $(2,0)$ e Taylor. 
Lema 2.7. Seja $\gamma: I \rightarrow R^{2}$ uma curva Legendriana. Então as afirmações seguintes são equivalentes:

1. $\nu^{\prime}(t) \neq 0, \forall t \in I$.

2. $\gamma$ pode ser parametrizada pelo ângulo $\theta$ que suas tangentes fazem com uma direção fixa.

Para uma demonstração, ver [6].

Nesse trabalho iremos considerar curvas Legendrianas com curvatura não nula, que satisfaçam uma das afirmações do Lema (2.7). Se $\gamma$ é fechada, é chamada de porco espinho, hedgehog em inglês. Iremos representar o conjunto de curvas legendrianas fechadas com curvatura não nula que satisfazem o lema (2.7) e possuem período $2 \pi$ pela letra $\mathscr{H}$.

Definição 2.8 (Rosáceas). Iremos representar o conjunto de curvas legendrianas fechadas com curvatura não nula que satisfazem o lema (2.7) e possuem período $2 m \pi, m$ inteiro positivo, pela letra $\mathscr{H}_{m}$. Chamamos essas curvas de $m$-rosáceas ou $m$-hedghogs.

Definição 2.9. Considere $\gamma \in \mathscr{H}_{m}$. Definimos um n-cúspide ou cúspide não ordinário uma singularidade da forma $(n+1) / n$.

\section{4}

\section{Círculo Unitário Dual, Função Suporte e raio de Curvatura}

Seja $U \subset \mathbb{R}^{2}$ bola unitária de Minkowski. Vamos assumir $U$ quadraticamente convexa, isto é, com curvatura estritamente positiva e fronteira $u$ suave. Considere $u$ parametrizada por $u(\theta), \theta \in[0,2 \pi], u(\theta+\pi)=$ $-u(\theta)$, onde $\theta$ é o ângulo que a tangente em $u$ faz com uma direção fixa. Com essas hipóteses teremos $\left[u^{\prime}(\theta), u^{\prime \prime}(\theta)\right]>0$ e $\left[u(\theta), u^{\prime}(\theta)\right]>0$.

A bola unitária dual $U^{*}$ pode ser identificada com o conjunto convexo $V$ do plano, onde, a parametrização da fronteira de $V$ é

$$
v(\theta)=\frac{u^{\prime}(\theta)}{\left[u(\theta), u^{\prime}(\theta)\right]}
$$

Dessa forma teremos $[u(\theta), v(\theta)]=1$ e $\left[u^{\prime}(\theta), v(\theta)\right]=0$. Pode-se mostrar que $V$ é convexa e simétrica, $\left[v(\theta), v^{\prime}(\theta)\right]>0,\left[v^{\prime}(\theta), v^{\prime \prime}(\theta)\right]>0$ e $v(\theta+\pi)=-v(\theta)$. Além disso

$$
u(\theta)=-\frac{v^{\prime}(\theta)}{\left[v(\theta), v^{\prime}(\theta)\right]},
$$


o que mostra que o dual do dual é ele próprio.

Definição 2.10. Seja $\gamma \in \mathscr{H}$ curva simples, fechada, com curvatura estritamente positiva. A esse tipo de curva chamaremos de oval.

Definição 2.11. Para $\gamma \in \mathscr{H}$ definimos a função real $h(\theta)=[\gamma(\theta), v(\theta)]$ que é chamada de $U$ - função suporte. Onde v é a parametrização da fronteira de $V=U^{*}$.

Diferenciando $h(\theta)=[\gamma(\theta), v(\theta)]$ nós obtemos

$$
\begin{aligned}
h^{\prime}(\theta) & =\left[\gamma^{\prime}(\theta), v(\theta)\right]+\left[\gamma(\theta), v^{\prime}(\theta)\right] \\
& =\left[\gamma(\theta), v^{\prime}(\theta)\right] \\
& =\left[\gamma(\theta),-u(\theta)\left[v(\theta), v^{\prime}(\theta)\right]\right] \\
& =[u(\theta), \gamma(\theta)] \cdot\left[v(\theta), v^{\prime}(\theta)\right]
\end{aligned}
$$

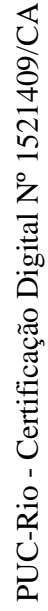

Assim podemos escrever

$$
\gamma(\theta)=h(\theta) u(\theta)+\frac{h^{\prime}(\theta)}{\left[v(\theta), v^{\prime}(\theta)\right]} v(\theta) .
$$

Diferenciando (2.3) teremos

$$
\gamma^{\prime}(\theta)=\left(h+\frac{1}{\left[u, u^{\prime}\right]}\left(\frac{h^{\prime}}{\left[v, v^{\prime}\right]}\right)^{\prime}\right)(\theta) u^{\prime}(\theta) .
$$

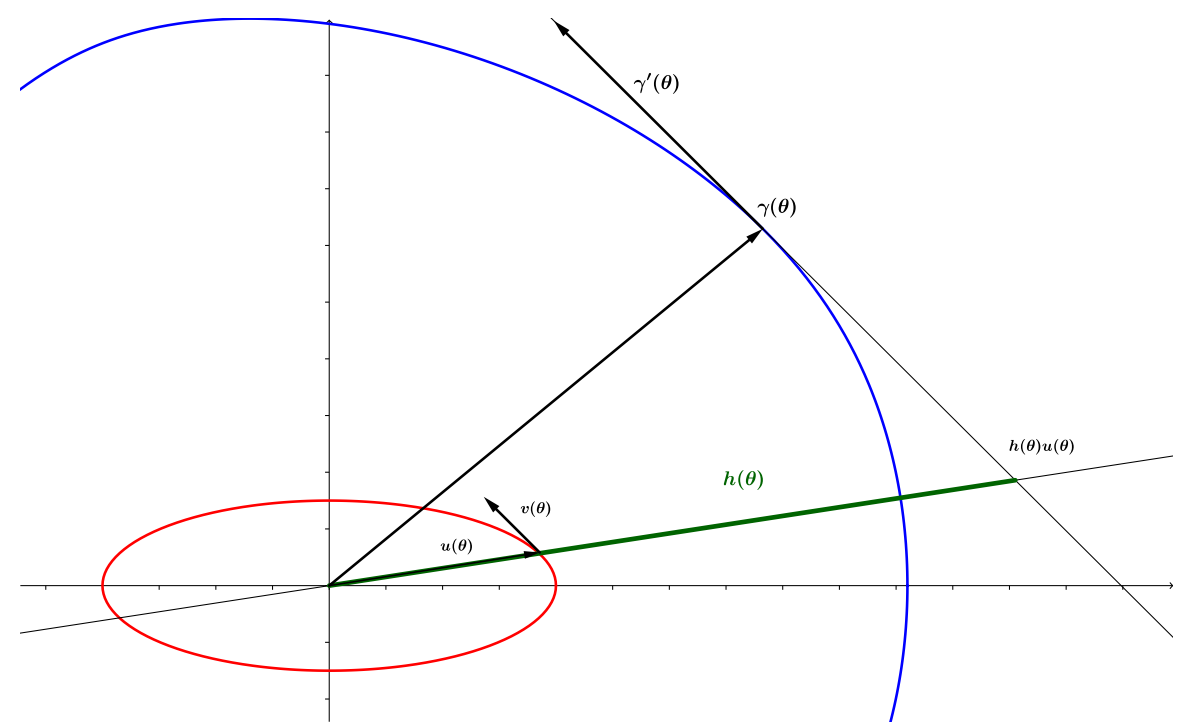

Figura 2.5: Interpretação geométrica da $U$-função suporte 
Geometricamente a $U$-função suporte $h(\theta)$ de $\gamma(\theta)$ é obtida pela interseção da reta tangente, em $\theta$, com a reta $\{t u(\theta), t \in \mathbb{R}\}$. Essas retas se interceptam no ponto $h(\theta) u(\theta)$. Ver figura 2.5 .

Definição 2.12. Definimos o raio de curvatura $r(\theta)$ de $\gamma(\theta) \in \mathscr{H}$ pela condição

$$
\gamma^{\prime}(\theta)=r(\theta) u^{\prime}(\theta)
$$

Usando (2.4) teremos

$$
r(\theta)=h(\theta)+\frac{1}{\left[u, u^{\prime}\right]}\left(\frac{h^{\prime}}{\left[v, v^{\prime}\right]}\right)^{\prime}(\theta) .
$$

\section{5}

\section{Exemplo}

Exemplo 2.13. Considere o exemplo $(2.2)$ onde $u(t)=\left((\cos t)^{2 / 3},(\sin t)^{2 / 3}\right), 0 \leq$ $t \leq 2 \pi$ é o círculo unitário.

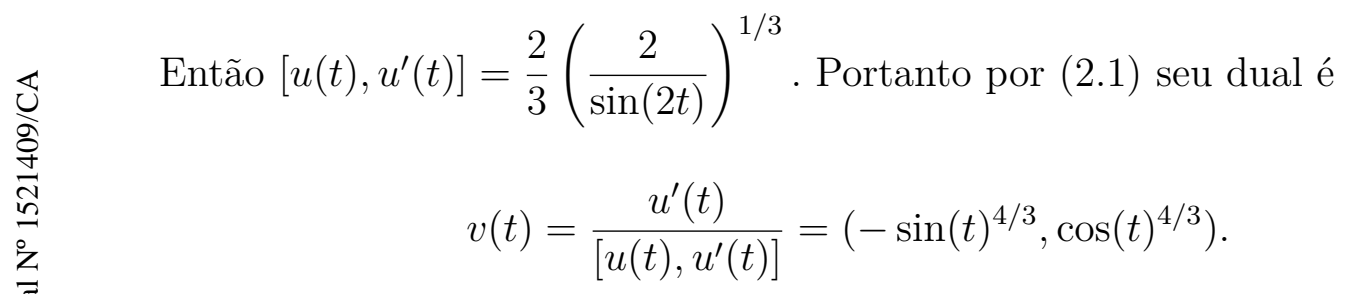

Nesse exemplo $u$ não é quadraticamente convexa, porém mesmo assim podemos calcular seu dual pela fórmula (2.1). Ver figura 2.6.

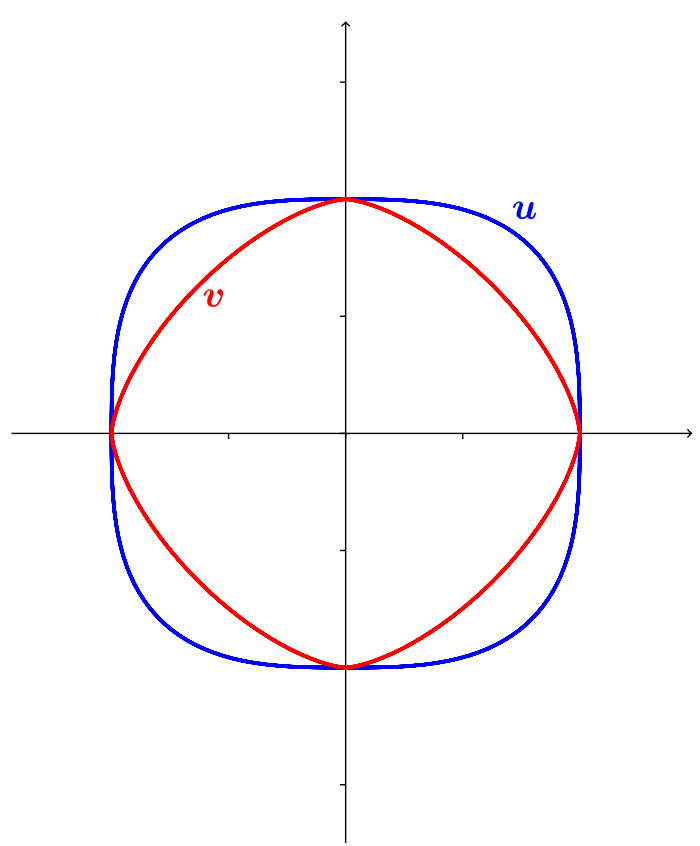

Figura 2.6: Bola unitária $U$ e seu dual $V$ 


\section{3 \\ Cáustica de Wigner e Conjunto de Medida de Largura Constante}

Seja $\gamma \in \mathscr{H}$, isto é, fechada, suave e parametrizada por $\theta \in[0,2 \pi]$. Considere $\left(\mathbb{R}^{2},\|\cdot\|_{U}\right)$.

Definição 3.1 (Paralelos). Chamamos de paralelo de $\gamma(\theta)$ a curva

$$
\gamma_{c}(\theta)=\gamma(\theta)+c u(\theta) ; c \in \mathbb{R}
$$

O raio de curvatura de $\gamma_{c}(\theta)$ é $r(\theta)+c$, pois $\gamma_{c}^{\prime}(\theta)=(r(\theta)+c) u^{\prime}(\theta)$, onde $r(\theta)$ é o raio de curvatura de $\gamma(\theta)$. Os cúspides dos paralelos ocorrem quando $r(\theta)+c$ troca de sinal. Observe que $\left[\gamma_{c}^{\prime}(\theta), \gamma_{c}^{\prime \prime}(\theta)\right]=(r(\theta)+c)^{2}\left[u^{\prime}(\theta), u^{\prime \prime}(\theta)\right]$. Assim concluímos que $\gamma_{c}$ é convexa fora dos cúspides. Em particular $\gamma_{c}$ é estritamente convexa, oval, para $c>\sup _{\theta \in[0,2 \pi]}|r(\theta)|$. A função suporte de um paralelo é

$$
h_{\gamma_{c}}(\theta)=\left[\gamma_{c} \theta, v(\theta)\right]=[\gamma(\theta)+c u(\theta), v(\theta)]=h(\theta)+c .
$$

Definição 3.2 (Comprimento-v com sinal). Seja $\gamma \in \mathscr{H}$ curva parametrizada por $\theta$. Como $\gamma^{\prime}(\theta)=r(\theta)\left[u, u^{\prime}\right](\theta) v(\theta)$, definimos comprimento-v com sinal

$$
L_{v}(\gamma)=\int_{0}^{2 \pi} r(\theta)\left[u(\theta), u^{\prime}(\theta)\right] d \theta
$$

onde $r(\theta)$ é o raio de curvatura de $\gamma(\theta)$.

A partir dessa definição podemos ter comprimento-v com sinal igual a zero.

Definição 3.3 (Área com sinal). Seja $\gamma \in \mathscr{H}$ curva parametrizada por $\theta$. Definimos área com sinal de $\gamma$ como

$$
\widetilde{\mathrm{A}}(\gamma)=\frac{1}{2} \int_{0}^{2 \pi}\left[\gamma(\theta), \gamma^{\prime}(\theta)\right] d \theta .
$$

A partir dessa definição podemos ter área com sinal com valor negativo. Importante notar que se $\gamma$ é uma oval centrada na origem e orientada positivamente então a área com sinal é a própria área limitada pela curva. 
Capítulo 3. Cáustica de Wigner e Conjunto de Medida de Largura Constante22

\section{1}

\section{Cáustica De Wigner e Curvas de Largura Constante}

Definição 3.4. Seja $\gamma \in \mathscr{H}$. Chamamos Evoluta de Área ou Cáustica de Wigner $(C W)$ o conjunto

$$
C W(\gamma)(\theta)=\frac{\gamma(\theta)+\gamma(\theta+\pi)}{2} ; \theta \in[0,2 \pi]
$$

Como $C W(\gamma)(\theta)=C W(\gamma)(\theta+\pi)$ temos que $C W(\gamma)(\theta)$ possui périodo $\pi$ e dá

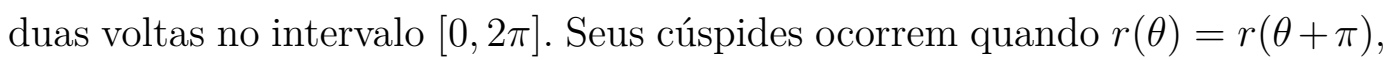
pois $C W^{\prime}(\gamma)(\theta)=\left(\frac{r(\theta)-r(\theta+\pi)}{2}\right) u^{\prime}(\theta)$. A função suporte de $C W(\gamma)(\theta)$ é

$$
h_{C W(\gamma)}=[(\gamma(\theta)+\gamma(\theta+\pi)) / 2, v(\theta)]=\frac{h(\theta)}{2}-\frac{h(\theta+\pi)}{2} .
$$

Definição 3.5 (Largura Constante). Dizemos que uma curva $\gamma \in \mathscr{H}$ possui largura constante se, $h(\theta)+h(\theta+\pi)=2 d, d>0(d$ constante real $)$. Nesse caso dizemos que a largura da curva $\gamma$ é $2 d$.

Geometricamente uma curva tem largura constante se a distância, na $U$-norma, entre as retas tangentes paralelas é sempre a mesma.

Exemplo 3.6. Considere a curva $\gamma \in \mathscr{H}$ tal que $h(\theta)=\cos (\theta)+\cos (3 \theta)+15$.

Ver figura 3.1.

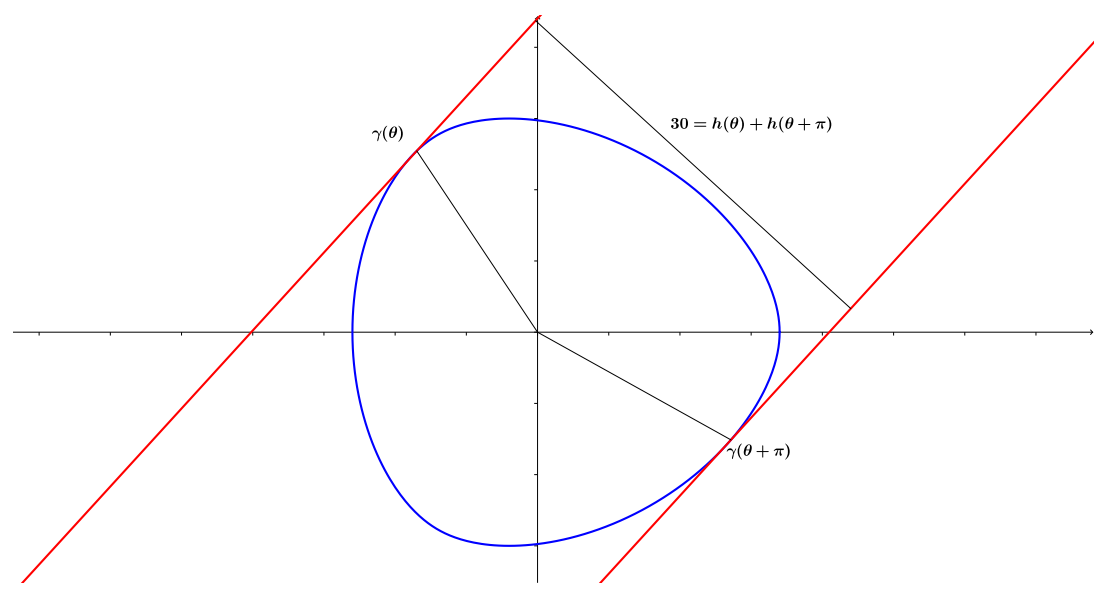

Figura 3.1: Interpretação geométrica de curva $\gamma$ com largura constante.

Proposição 3.7. $\gamma \in \mathscr{H}$ tem largura constante $\Leftrightarrow \gamma(\theta)-\gamma(\theta+\pi)=2 d u(\theta), d$ constante.

Demonstração. $(\Rightarrow)$ 
Capítulo 3. Cáustica de Wigner e Conjunto de Medida de Largura Constante23

Da equação (2.3) temos

$$
\gamma(\theta)=h(\theta) u(\theta)+\frac{h^{\prime}(\theta)}{\left[v(\theta), v^{\prime}(\theta)\right]} v(\theta)
$$

assim teremos

$$
\begin{aligned}
& \gamma(\theta)-\gamma(\theta+\pi)=(h(\theta)+h(\theta+\pi)) u(\theta)+\frac{(h(\theta)+h(\theta+\pi))^{\prime}}{\left[v(\theta), v^{\prime}(\theta)\right]} v(\theta) \\
& \gamma(\theta)-\gamma(\theta+\pi)=2 d u(\theta) \\
& (\Leftarrow) \\
& \qquad \begin{aligned}
h(\theta)+h(\theta+\pi) & =[\gamma(\theta), v(\theta)]+[\gamma(\theta+\pi), v(\theta+\pi)] \\
& =[\gamma(\theta)-\gamma(\theta+\pi), v(\theta)] \\
& =[2 d u(\theta), v(\theta)] \\
& =2 d(\text { constante })
\end{aligned}
\end{aligned}
$$

Definição 3.8 (Largura Média). Chamamos de largura média da curva $\gamma \in \mathscr{H}$ número $\bar{w}_{\gamma}=\frac{L_{v}(\gamma)}{A(U)}$, onde $L_{v}(\gamma)$ é o comprimento da curva $\gamma$ na norma dual e $A(U)$ é a área da bola unitária $U$.

Teorema 3.9 (Barbier). Seja $\gamma \in \mathscr{H}$ com largura constante $2 d$ então $L_{v}(\gamma)=2 d A(U)$, onde $U$ é a bola unitária.

\section{Demonstração.}

$$
\begin{aligned}
L_{v}(\gamma) & =\int_{0}^{2 \pi} r(\theta)\left[u(\theta), u^{\prime}(\theta)\right] d \theta=\int_{0}^{2 \pi}\left[u(\theta), \gamma^{\prime}(\theta)\right] d \theta \\
& =\int_{0}^{2 \pi}\left[\gamma(\theta), u^{\prime}(\theta)\right] d \theta=\frac{1}{2} \int_{0}^{2 \pi}\left[\gamma(\theta), u^{\prime}(\theta)\right]+\left[\gamma(\theta+\pi), u^{\prime}(\theta+\pi)\right] d \theta \\
& =\frac{1}{2} \int_{0}^{2 \pi}(h(\theta)+h(\theta+\pi))\left[u(\theta), u^{\prime}(\theta)\right] d \theta \\
& =2 d \frac{1}{2} \int_{0}^{2 \pi}\left[u(\theta), u^{\prime}(\theta)\right] d \theta=2 d A(U)
\end{aligned}
$$

Caso a curva $\gamma$ possua largura constante $2 d$ então pelo Teorema de Barbier, $L_{v}(\gamma)=A(U) 2 d$ (ver [16] e [18]). Nesse caso a largura média e a largura da curva são iguais. 
Proposição 3.10. Seja $\gamma \in \mathscr{H}$. CW é paralelo de $\gamma \Leftrightarrow \gamma$ tem largura constante.

\section{Demonstração. $(\Rightarrow)$}

$$
(\gamma(\theta)+\gamma(\theta+\pi)) / 2=\gamma(\theta)+c u(\theta) \Rightarrow \gamma(\theta)-\gamma(\theta+\pi)=-2 c u(\theta) \text {. Pela }
$$
proposição (3.7) $\gamma$ tem largura constante.

$(\Leftarrow)$

$$
\begin{aligned}
C W(\gamma)(\theta) & =\frac{\gamma(\theta)+\gamma(\theta+\pi)}{2} \\
& =\gamma(\theta)-\frac{\gamma(\theta)-\gamma(\theta+\pi)}{2} \\
& =\gamma(\theta)-\frac{c}{2} u(\theta)
\end{aligned}
$$

Proposição 3.11. Considere $\gamma \in \mathscr{H}$ parametrizada por $\theta . C W(\gamma)=\{0\} \Leftrightarrow \gamma$ é simétrica com respeito a origem.

\section{Demonstração.}

$$
C W(\gamma)(\theta)=\frac{\gamma(\theta)+\gamma(\theta+\pi)}{2}=\frac{\gamma(\theta)-\gamma(\theta)}{2}=0
$$

Proposição 3.12. $C W(C W(\gamma)(\theta))=C W(\gamma)(\theta)$

Demonstração.

$$
\begin{aligned}
C W(C W(\gamma)(\theta)) & =\frac{C W(\gamma)(\theta)+C W(\gamma)(\theta+\pi)}{2} \\
& =\frac{2 C W(\gamma)(\theta)}{2} \\
& =C W(\gamma)(\theta)
\end{aligned}
$$

Proposição 3.13. $\gamma \in \mathscr{H} . C W\left(\gamma_{c}\right)=C W(\gamma)$

Demonstração.

$$
\begin{aligned}
C W\left(\gamma_{c}\right)(\theta) & =\frac{\gamma(\theta)+c u(\theta)+\gamma(\theta+\pi)+c u(\theta+\pi)}{2} \\
& =\frac{\gamma(\theta)+\gamma(\theta+\pi)}{2} \\
& =C W(\gamma)
\end{aligned}
$$


Capítulo 3. Cáustica de Wigner e Conjunto de Medida de Largura Constante25

Proposição 3.14. $\gamma \in \mathscr{H} . C W(\gamma)$ tem comprimento-v com sinal igual a zero.

\section{Demonstração.}

$$
\begin{aligned}
L_{v}(C W(\gamma)) & =\int_{0}^{2 \pi} r_{C W}(\theta)\left[u(\theta), u^{\prime}(\theta)\right] d \theta \\
& =\int_{0}^{2 \pi} \frac{(r(\theta)-r(\theta+\pi))}{2}\left[u(\theta), u^{\prime}(\theta)\right] d \theta \\
& =\frac{1}{2}\left[\int_{0}^{2 \pi} r(\theta)\left[u(\theta), u^{\prime}(\theta)\right] d \theta-\int_{0}^{2 \pi} r(\theta+\pi)\left[u(\theta), u^{\prime}(\theta)\right] d \theta\right] \\
& =\frac{1}{2}\left[\int_{0}^{2 \pi} r(\theta)\left[u(\theta), u^{\prime}(\theta)\right] d \theta-\int_{0}^{2 \pi} r(\theta+\pi)\left[u(\theta+\pi), u^{\prime}(\theta+\pi)\right] d \theta\right] \\
& =\frac{1}{2}\left[L_{v}(\gamma)-L_{v}(\gamma)\right]=0
\end{aligned}
$$

\section{2}

\section{Conjunto de Medida de Largura Constante e Curvas Simétricas}

Definição 3.15. Considere $\gamma \in \mathscr{H}$. Definimos como Conjunto de Medida de Largura constante, abreviado por CMLC,

$$
C M L C(\gamma)(\theta)=\frac{1}{2}\left(\gamma(\theta)-\gamma(\theta+\pi)-\bar{w}_{\gamma} u(\theta)\right), \theta \in[0,2 \pi]
$$

Ver [23] para versão Euclidiana do CMLC.

A função suporte de $C M L C$ é

$$
h_{C M L C(\gamma)}(\theta)=\frac{1}{2}\left[\gamma(\theta)-\gamma(\theta+\pi)-\bar{w}_{\gamma} u(\theta), v(\theta)\right]=\frac{1}{2}\left(h(\theta)+h(\theta+\pi)-\bar{w}_{\gamma}\right) .
$$

Proposição 3.16. Seja $\gamma \in \mathscr{H}$. A curva $C M L C(\gamma)(\theta)$ é simétrica.

Demonstração.

$$
\begin{aligned}
C M L C(\gamma)(\theta+\pi) & =\frac{\gamma(\theta+\pi)-\gamma(\theta+2 \pi)-\bar{w}_{\gamma} u(\theta+\pi)}{2} \\
& =\frac{\gamma(\theta+\pi)-\gamma(\theta)+\bar{w}_{\gamma} u(\theta)}{2} \\
& =-\frac{\gamma(\theta)-\gamma(\theta+\pi)-\bar{w}_{\gamma} u(\theta)}{2} \\
& =-C M L C(\gamma)(\theta) .
\end{aligned}
$$


Capítulo 3. Cáustica de Wigner e Conjunto de Medida de Largura Constante26

Proposição 3.17. Seja $\gamma \in \mathscr{H}$. CMLC é paralelo de $\gamma \Leftrightarrow \gamma$ é simétrica.

\section{Demonstração.}

$(\Rightarrow)$

$$
\begin{aligned}
C M L C(\gamma)(\theta) & =\gamma(\theta)+c u(\theta) \Rightarrow \\
\gamma(\theta) & =C M L C(\gamma)(\theta)-c u(\theta) \Rightarrow \\
\gamma(\theta+\pi) & =C M L C(\gamma)(\theta+\pi)-c u(\theta+\pi)=-\gamma(\theta) .
\end{aligned}
$$

$(\Leftarrow)$

$$
\begin{aligned}
C M L C(\gamma)(\theta) & =\frac{\gamma(\theta)-\gamma(\theta+\pi)-\bar{w}_{\gamma} u(\theta)}{2} \\
& =\frac{\gamma(\theta)+\gamma(\theta)-\bar{w}_{\gamma} u(\theta)}{2} \\
& =\gamma(\theta)-\frac{\bar{w}_{\gamma}}{2} u(\theta)
\end{aligned}
$$

Portanto $C M L C$ é paralelo de $\gamma$ onde $c=-\frac{\bar{w}_{\gamma}}{2}$

Teorema 3.18. Considere $\gamma \in \mathscr{H}$ parametrizada por $\theta . C M L C(\gamma)=\{0\} \Leftrightarrow$ $\gamma$ possui largura constante.

Demonstração. $(\Rightarrow)$ Por hipótese $\gamma(\theta)=\gamma(\theta+\pi)+\bar{w}_{\gamma} u(\theta)$

$$
\begin{aligned}
h(\theta)+h(\theta+\pi) & = \\
& =[\gamma(\theta), v(\theta)]+[\gamma(\theta+\pi), v(\theta+\pi)] \\
& =\left[\gamma(\theta+\pi)+\bar{w}_{\gamma} u(\theta), v(\theta)\right]+[\gamma(\theta+\pi),-v(\theta)] \\
& =[\gamma(\theta+\pi), v(\theta)]-[\gamma(\theta+\pi), v(\theta)]+\left[\bar{w}_{\gamma} u(\theta), v(\theta)\right] \\
& =\bar{w}_{\gamma}[u(\theta), v(\theta)]=\bar{w}_{\gamma}(\text { constante })
\end{aligned}
$$

$(\Leftarrow)$ Por hipótese $\bar{w}_{\gamma}$ é a largura de $\gamma$. Utilizando a equação (3.3) teremos

$$
\begin{aligned}
\gamma(\theta)-\gamma(\theta+\pi) & = \\
& =(h(\theta)+h(\theta+\pi)) u(\theta)+(h(\theta)+h(\theta+\pi))^{\prime} \frac{v(\theta)}{\left[v(\theta), v^{\prime}(\theta)\right]} \\
& =(h(\theta)+h(\theta+\pi)) u(\theta) \\
& =\bar{w}_{\gamma} u(\theta)
\end{aligned}
$$


Proposição 3.19. $\gamma \in \mathscr{H}$. CMLC tem comprimento-v zero.

Demonstração.

Como $(C M L C)^{\prime}(\gamma)(\theta)=\left(\frac{r(\theta)+r(\theta+\pi)-\bar{w}_{\gamma}}{2}\right) u^{\prime}(\theta)$ temos

$$
\begin{aligned}
L_{v}(C M L C(\gamma)) & =\int_{0}^{2 \pi}\left(\frac{r(\theta)+r(\theta+\pi)-\bar{w}_{\gamma}}{2}\right)\left[u(\theta), u^{\prime}(\theta)\right] d \theta \\
& =\frac{1}{2}\left(2 L_{v}(\gamma)-2 \bar{w}_{\gamma} A(U)\right) \\
& =L_{v}(\gamma)-\frac{L_{v}(\gamma)}{A(U)} A(U)=0
\end{aligned}
$$

Proposição 3.20. $\gamma \in \mathscr{H} . C M L C(C M L C(\gamma))=C M L C(\gamma)$

\section{Demonstração.}

Como CMLC é simétrico e tem comprimento-v zero. temos

$$
\begin{aligned}
C M L C(C M L C(\gamma)(\theta)) & =\frac{C M L C(\gamma)(\theta)-C M L C(\gamma)(\theta+\pi)-\bar{w}_{C M L C} u(\theta)}{2} \\
& =\frac{C M L C(\gamma)(\theta)+C M L C(\gamma)(\theta)-0 \cdot u(\theta)}{2} \\
& =C M L C(\gamma)(\theta)
\end{aligned}
$$

Proposição 3.21. $\gamma \in \mathscr{H} . C M L C\left(\gamma_{c}\right)=C M L C(\gamma)$

Demonstração. Como $L_{v}\left(\gamma_{c}\right)=\int_{0}^{2 \pi}(r(\theta)+c)\left[u(\theta), u^{\prime}(\theta)\right] d \theta=L_{v}(\gamma)+$ $2 c A(U)$. Dessa forma $\bar{w}_{\gamma_{c}}=\bar{w}_{\gamma}+2 c$. Assim teremos

$$
\begin{aligned}
C M L C\left(\gamma_{c}\right)(\theta) & =\frac{\left.\gamma(\theta)+c u(\theta)-(\gamma(\theta+\pi)+c u(\theta+\pi))-\bar{w}_{\gamma_{c}} u(\theta)\right)}{2} \\
& =\frac{\gamma(\theta)-\gamma(\theta+\pi)+2 c u(\theta)-\left(\bar{w}_{\gamma}+2 c\right) u(\theta)}{2} \\
& =\frac{\gamma(\theta)-\gamma(\theta+\pi)-\bar{w}_{\gamma} u(\theta)}{2} \\
& =C M L C(\gamma)(\theta)
\end{aligned}
$$




\section{3 \\ Decomposição da curva $\gamma$ como soma de Caústica de Wigner, CMLC e bola unitária.}

Proposição 3.22. $\gamma \in \mathscr{H} \cdot \gamma(\theta)=C W(\gamma)(\theta)+C M L C(\gamma)(\theta)+\frac{\bar{w}_{\gamma}}{2} u(\theta) e$ $h_{\gamma}(\theta)=h_{C W(\gamma)}(\theta)+h_{C M L C(\gamma)}(\theta)+\frac{\bar{w}_{\gamma}}{2}$.

Corolário 3.23. $\gamma \in \mathscr{H} \cdot \gamma(\theta)=C W(\gamma)(\theta)+C M L C(\gamma)(\theta)$ e $h_{\gamma}(\theta)=$ $h_{C W(\gamma)}(\theta)+h_{C M L C(\gamma)}(\theta) \Leftrightarrow L_{V}(\gamma)=0$

É simples ver que os resultados acima são consequência do fato que

$$
\gamma(\theta)=\frac{\gamma(\theta)+\gamma(\theta+\pi)}{2}+\frac{\gamma(\theta)-\gamma(\theta+\pi)}{2}
$$

\section{4}

\section{Cuspides CMLC e Cáustica de Wigner}

Teorema 3.24. Seja $\gamma \in \mathscr{H}$ curva que possui apenas singularidades ordinárias, isto é, do tipo 2-cúspide. Então o número de 2-cúspides de $C M L C(\gamma)$ é múltiplo de quatro.

Demonstração. O número de cúspides de $C M L C$ é igual ao número de zeros de $r_{C M L C(\gamma)}(\theta)=\frac{1}{2} r(\theta)+r(\theta+\pi)-\bar{w}_{\gamma}$ no intervalo de 0 até $2 \pi$. Como $r_{C M L C(\gamma)}(0)=r_{C M L C(\gamma)}(\pi)$ então o número de cúspides de 0 a $\pi$ é par. Como $r_{C M L C(\gamma)}(\theta)$ é $\pi$-periódica concluímos que o números de cúspides deve ser múltiplo de quatro.

Teorema 3.25. Seja $\gamma \in \mathscr{H}$ curva que possui apenas singularidades ordinárias, isto é, do tipo 2-cúspide. Então o número de 2-cúspides de $C W(\gamma)$ é $2 k, k$ impar.

Demonstração. Observe que $C W^{\prime}(\gamma)(\theta)=\frac{r(\theta)-r(\theta+\pi)}{2} u^{\prime}(\theta)$. Assim, $r_{C W(\gamma)}(\theta)=\frac{r(\theta)-r(\theta+\pi)}{2}$. Como $r_{C W(\gamma)}(\theta)=-r_{C W(\gamma)}(\theta+\pi)$, o número de zeros de $\theta$ até $\theta+\pi$ é ímpar. Como $C W$ é $\pi$-periódica temos o desejado.

Os teoremas acimas são falsos se considerarmos singularidades do tipo $n$-cúspides com $n \neq 2$. Basta observar que a curva do exemplo 2.6 é uma Cáustica de Wigner que possui 8 cúspides no intervalo [0,2 $2 \pi$. 
Capítulo 3. Cáustica de Wigner e Conjunto de Medida de Largura Constante29

\section{5}

\section{Áreas com sinal de CW e CMLC}

Definição 3.26. Sejam $\gamma_{0}$ e $\gamma_{1}$ ovais parametrizadas por $\theta$. Definimos como área mista das curvas $\gamma_{0}$ e $\gamma_{1}$,

$$
A\left(\gamma_{0}, \gamma_{1}\right)=\frac{1}{2} \int_{0}^{2 \pi}\left[\gamma_{0}, \gamma_{1}^{\prime}\right](\theta) d \theta
$$

(para mais detalhes ver [8]).

Teorema 3.27 (Desigualdade de Minkowski). Sejam $\gamma_{0}$ e $\gamma_{1}$ ovais parametrizadas por $\theta$. Então

$$
A\left(\gamma_{0}, \gamma_{1}\right)^{2} \geq A\left(\gamma_{0}\right) A\left(\gamma_{1}\right)
$$

onde $A\left(\gamma_{0}\right), A\left(\gamma_{1}\right)$ e $A\left(\gamma_{0}, \gamma_{1}\right)$ são respectivamente áreas das curvas e área mista.

Para prova ver [21]. Interessante notar que fazendo $\gamma_{0}=\gamma$ e $\gamma_{1}=u, u$ bola unitária, teremos pela equação 3.4

$$
\begin{aligned}
A(\gamma, u)^{2} & =\left[\frac{1}{2} \int_{0}^{2 \pi}\left[\gamma(\theta), u^{\prime}(\theta)\right] d \theta\right]^{2}=\left[\frac{1}{2} \int_{0}^{2 \pi} r(\theta)\left[u(\theta), u^{\prime}(\theta)\right] d \theta\right]^{2} \\
& =\frac{L_{v}^{2}(\gamma)}{4} \geq A(u) A(\gamma)
\end{aligned}
$$

isto é, desigualdade isoperimétrica de Minkowski,

$$
L_{v}^{2}(\gamma) \geq 4 A(u) A(\gamma)
$$

Proposição 3.28. Se a curva $\gamma \in \mathscr{H}$ parametrizada por $\theta$ então $\widetilde{\mathrm{A}}(C W(\gamma))<$ 0, $\tilde{\mathrm{A}}(C M L C(\gamma))<0$.

Demonstração. Ver seção (5.1) áreas e comprimentos. 
Capítulo 3. Cáustica de Wigner e Conjunto de Medida de Largura Constante30

\section{6}

\section{Evolutas}

Definição 3.29. Definimos Evoluta de $\gamma \in \mathscr{H}$ como sendo a curva $e_{\gamma}(\theta)=$ $\gamma(\theta)-r(\theta) u(\theta)$, onde $r(\theta)$ é o raio de curvatura de $\gamma$.

Proposição 3.30. Seja $\gamma \in \mathscr{H}$. Os paralelos de $\gamma$ e a curva $\gamma$ possuem a mesma Evoluta.

Demonstração. Como $\gamma_{c}^{\prime}(\theta)=(r(\theta)+c) u^{\prime}(\theta)$ temos que a Evoluta do paralelo é $e_{\gamma_{c}}(\theta)=\gamma_{c}(\theta)-(r(\theta)+c) u(\theta)=\gamma(\theta)+c u(\theta)-(r(\theta)+c) u(\theta)=e_{\gamma}(\theta)$

Proposição 3.31. A Evoluta possui comprimento-u com sinal igual a zero.

Demonstração. Basta observar que $e_{\gamma}^{\prime}(\theta)=-r^{\prime}(\theta) u(\theta)$. Assim temos

$$
L_{u}\left(e_{\gamma}\right)=\int_{0}^{2 \pi}-r^{\prime}(\theta) d \theta=0
$$

Proposição 3.32. Seja $\gamma \in \mathscr{H}$. A v-função suporte da evoluta de $\gamma$ é $h_{e_{\gamma}}(\theta)=-\frac{h^{\prime}(\theta)}{\left[v(\theta), v^{\prime}(\theta)\right]}$, onde $h(\theta)$ é a u-função suporte de $\gamma$.

Demonstração. Considere $r(\theta)$ o raio de curvatura de $\gamma(\theta)$. Como $e_{\gamma}(\theta)=$ $\gamma(\theta)-r(\theta) u(\theta)$ então $e_{\gamma}^{\prime}(\theta)=-r^{\prime}(\theta) u(\theta)=\frac{r(\theta)}{\left[v(\theta), v^{\prime}(\theta)\right]} v^{\prime}(\theta)$.

$$
\begin{aligned}
h_{e_{\gamma}}(\theta) & =\left[e_{\gamma}(\theta), u(\theta)\right] \\
& =[\gamma(\theta)-r(\theta) u(\theta), u(\theta)] \\
& =[\gamma(\theta), u(\theta)] \\
& =\left[h(\theta) u(\theta)+\frac{h^{\prime}(\theta)}{\left[v(\theta), v^{\prime}(\theta)\right]} v(\theta), u(\theta)\right] \\
& =-\frac{h^{\prime}(\theta)}{\left[v(\theta), v^{\prime}(\theta)\right]}
\end{aligned}
$$


Proposição 3.33. Seja $\gamma \in \mathscr{H}$. A u-função suporte da dupla evoluta de $\gamma$ é

$$
h_{e_{e_{\gamma}}}(\theta)=-\frac{1}{\left[u(\theta), u^{\prime}(\theta)\right]}\left(\frac{h^{\prime}(\theta)}{\left[v(\theta), v^{\prime}(\theta)\right]}\right)^{\prime} \text {. }
$$

Onde $h(\theta)$ é a $u$-função suporte de $\gamma$.

Demonstração. Como $e_{e_{\gamma}}(\theta)=e_{\gamma}(\theta)-\frac{r(\theta)}{\left[v(\theta), v^{\prime}(\theta)\right]} v(\theta)$ então $e_{e_{\gamma}}^{\prime}(\theta)=$ $-\left(\frac{r(\theta)}{\left[v(\theta), v^{\prime}(\theta)\right]}\right)^{\prime} \frac{1}{[u(\theta), u(\theta)]} u^{\prime}(\theta)$.

$$
\begin{aligned}
h_{e_{e_{\gamma}}}(\theta) & =\left[e_{e_{\gamma}}(\theta), v(\theta)\right] \\
& =\left[e_{\gamma}(\theta)-\frac{r(\theta)}{\left[v(\theta), v^{\prime}(\theta)\right]} v(\theta), v(\theta)\right] \\
& =\left[e_{\gamma}(\theta), v(\theta)\right] \\
& =[\gamma(\theta)-r(\theta) u(\theta), v(\theta)] \\
& =h(\theta)-r(\theta) \\
& =h(\theta)-\left(h(\theta)+\frac{1}{\left[u(\theta), u^{\prime}(\theta)\right]}\left(\frac{h^{\prime}(\theta)}{\left[v(\theta), v^{\prime}(\theta)\right]}\right)^{\prime}\right) \\
& =-\frac{1}{\left[u(\theta), u^{\prime}(\theta)\right]}\left(\frac{h^{\prime}(\theta)}{\left[v(\theta), v^{\prime}(\theta)\right]}\right)^{\prime}
\end{aligned}
$$

Proposição 3.34. Seja $\gamma \in \mathscr{H}$. Então $e(C W(\gamma))=C W\left(e_{\gamma}\right)$ e $e(C M L C(\gamma))=C M L C\left(e_{\gamma}\right)$. Isto é, evolutas comutam com CW e CMLC.

\section{Demonstração.}

$$
\begin{aligned}
e_{C W}(\theta)= & C W(\theta)-r_{C W}(\theta) u(\theta) \\
= & \frac{\gamma(\theta)+\gamma(\theta+\pi)}{2}-\frac{r(\theta)-r(\theta+\pi)}{2} u(\theta) \\
= & \frac{\gamma(\theta)-r(\theta) u(\theta)+\gamma(\theta+\pi)-r(\theta+\pi) u(\theta+\pi)}{2} \\
= & \frac{e_{\gamma}(\theta)+e_{\gamma}(\theta+\pi)}{2} \\
& =C W\left(e_{\gamma}\right)(\theta)
\end{aligned}
$$




$$
\begin{aligned}
e_{C M L C}(\theta)= & C M L C(\theta)-r_{C M L C}(\theta) u(\theta) \\
= & \frac{\gamma(\theta)-\gamma(\theta+\pi)-\bar{w}_{\gamma} u(\theta)}{2}-\frac{r(\theta)+r(\theta+\pi)-\bar{w}_{\gamma}}{2} u(\theta) \\
= & \frac{\gamma(\theta)-r(\theta) u(\theta)-(\gamma(\theta+\pi)-r(\theta+\pi) u(\theta+\pi))-\bar{w}_{e_{\gamma}} u(\theta)}{2} \\
= & \frac{e_{\gamma}(\theta)-e_{\gamma}(\theta+\pi)-\bar{w}_{e_{\gamma}} u(\theta)}{2} \\
& \frac{e_{\gamma}(\theta)-e_{\gamma}(\theta+\pi)}{2} \\
= & C M L C\left(e_{\gamma}\right)(\theta)
\end{aligned}
$$

Observe que nesse caso $\bar{w}_{e_{\gamma}}=\frac{L_{u}\left(e_{\gamma}\right)}{A(u)}=0$.

\section{7}

\section{Exemplos}

Exemplo 3.35. Seja $h(\theta)=\cos (3 \theta)+20$ e $u(\theta)=(\cos (\theta), \sin (\theta))$. Como consequência $v(\theta)=(-\sin (\theta), \cos (\theta))$. Como

$$
\gamma(\theta)=h(\theta) u(\theta)+\frac{h^{\prime}(\theta)}{\left[v(\theta), v^{\prime}(\theta)\right]} v(\theta)
$$

teremos $\gamma(\theta)=(\cos (\theta) \cos (3 \theta)+3 \sin (\theta) \sin (3 \theta)+20 \cos (\theta), \cos (3 \theta) \sin (\theta)-$ $3 \sin (3 \theta) \cos (\theta)+20 \sin (\theta))$. A curva $\gamma(\theta)$ tem largura constante pois $h(\theta)+$ $h(\theta+\pi)=20$ dessa forma pelo teorema (3.16) $C M L C(\theta)=\{0\}$. Ver figura 3.2.

Exemplo 3.36. Seja $h(\theta)=\cos (4 \theta)+18$ e $u(\theta)=(\cos (\theta), \sin (\theta))$. Como consequência $v(\theta)=(-\sin (\theta), \cos (\theta))$. Como

$$
\gamma(\theta)=h(\theta) u(\theta)+\frac{h^{\prime}(\theta)}{\left[v(\theta), v^{\prime}(\theta)\right]} v(\theta)
$$

teremos $\gamma(\theta)=(\cos (\theta) \cos (4 \theta)+4 \sin (\theta) \sin (4 \theta)+18 \cos (\theta), \sin (\theta) \cos (4 \theta)-$ $4 \cos (\theta) \sin (4 \theta)+18 \sin (\theta))$.

A curva $\gamma(\theta)$ é simétrica pois $h(\theta)=h(\theta+\pi)$. Dessa forma teremos $C W(\gamma)(\theta)=\{0\}$ e por proposição (3.9) $C M L C(\gamma)(\theta)=\gamma(\theta)-18 u(\theta)$. Ver figura 3.3. 
Exemplo 3.37. Seja $h(\theta)=\cos (2 \theta)+\sin (3 \theta)+20$ e $u(\theta)=(\cos (\theta), \sin (\theta))$. Como consequência $v(\theta)=(-\sin (\theta), \cos (\theta))$. Como

$$
\gamma(\theta)=h(\theta) u(\theta)+\frac{h^{\prime}(\theta)}{\left[v(\theta), v^{\prime}(\theta)\right]} v(\theta)
$$

teremos $\gamma(\theta)=(\cos (\theta) \cos (2 \theta)+\cos (\theta) \sin (3 \theta)+2 \sin (\theta) \sin (2 \theta)-$ $3 \cos (3 \theta) \sin (\theta)+20 \cos (\theta), \cos (2 \theta) \sin (\theta)+\sin (3 \theta) \sin (\theta)-2 \sin (2 \theta) \cos (\theta)+$ $3 \cos (3 \theta) \cos (\theta)+20 \sin (\theta))$.

Nesse caso a oval $\gamma$ não é simétrica e nem possui largura constante.

$C W(\gamma)(\theta)=(\cos (\theta) \sin (3 \theta)-3 \sin (\theta) \cos (3 \theta), \sin (3 \theta) \sin (\theta)+3 \cos (3 \theta) \cos (\theta))$.

Como $L_{V}(\gamma)=40 \pi$ então

$C M L C(\gamma)(\theta)=(\cos (\theta) \cos (2 \theta)+2 \sin (2 \theta) \sin (\theta), \cos (2 \theta) \sin (\theta)-2 \sin (2 \theta) \cos (\theta))$

Ver figura 3.4

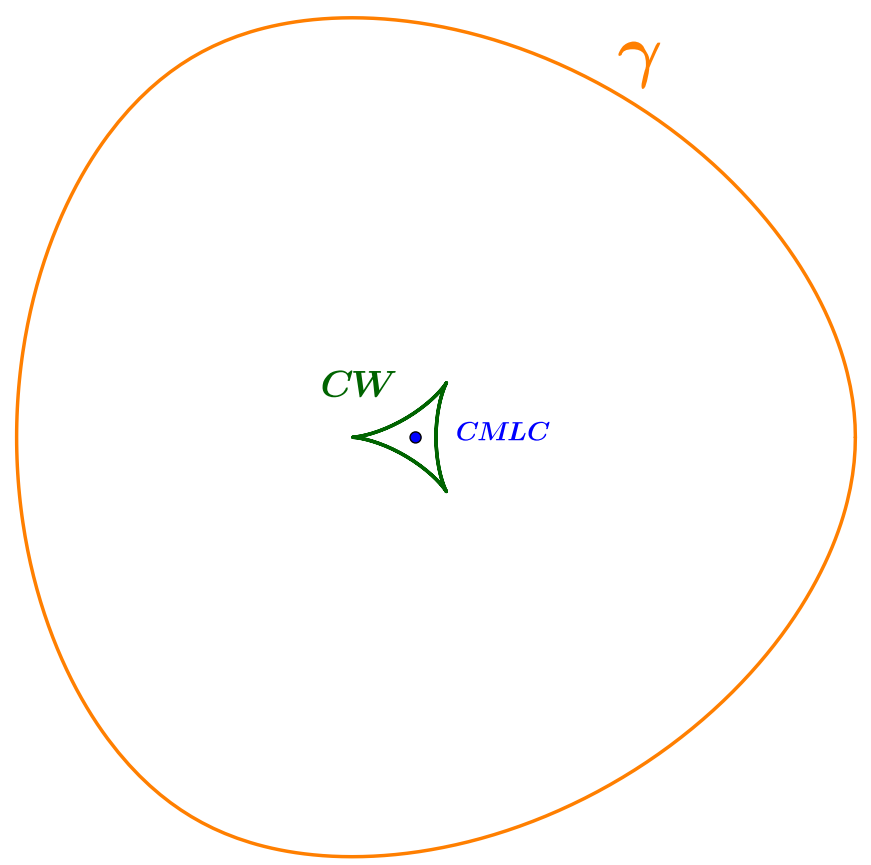

Figura 3.2: Curva $\gamma$ de largura constante, $C M L C(\gamma)$ e $C W(\gamma)$ 
Capítulo 3. Cáustica de Wigner e Conjunto de Medida de Largura Constante34

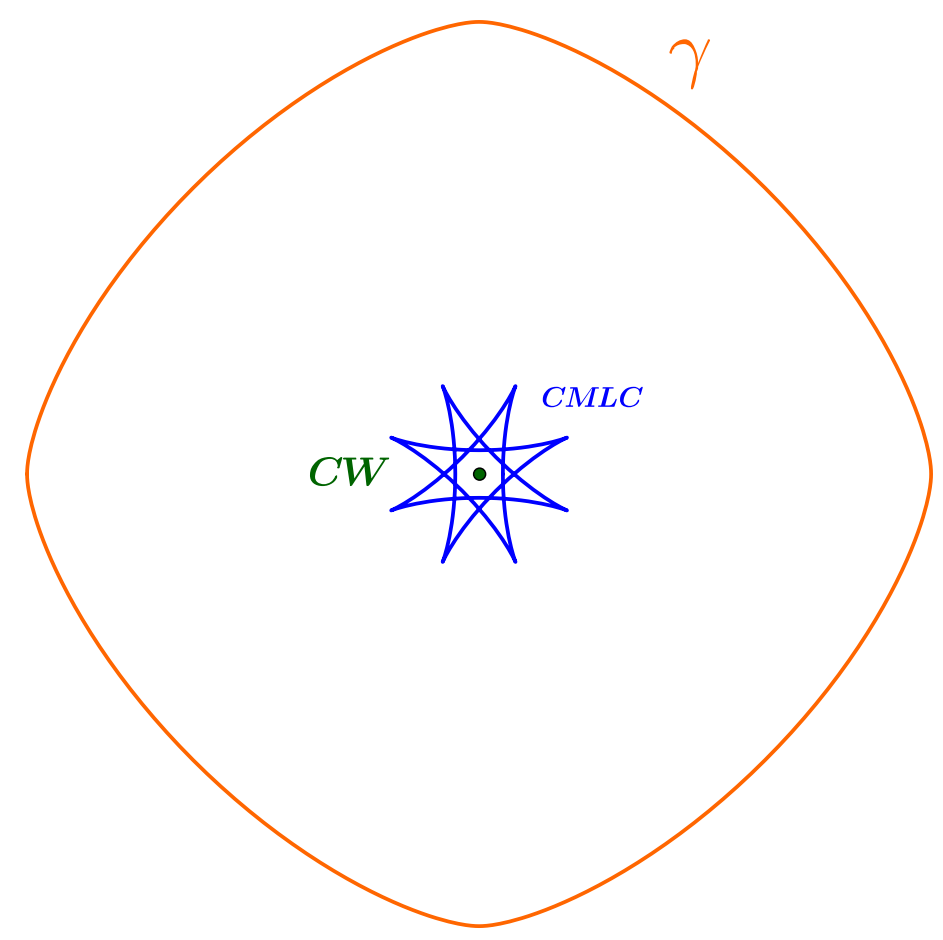

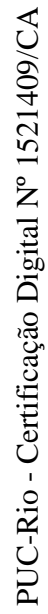

Figura 3.3: Curva $\gamma$ simétrica, Cáustica $C W(\gamma)$ e $C M L C(\gamma)$

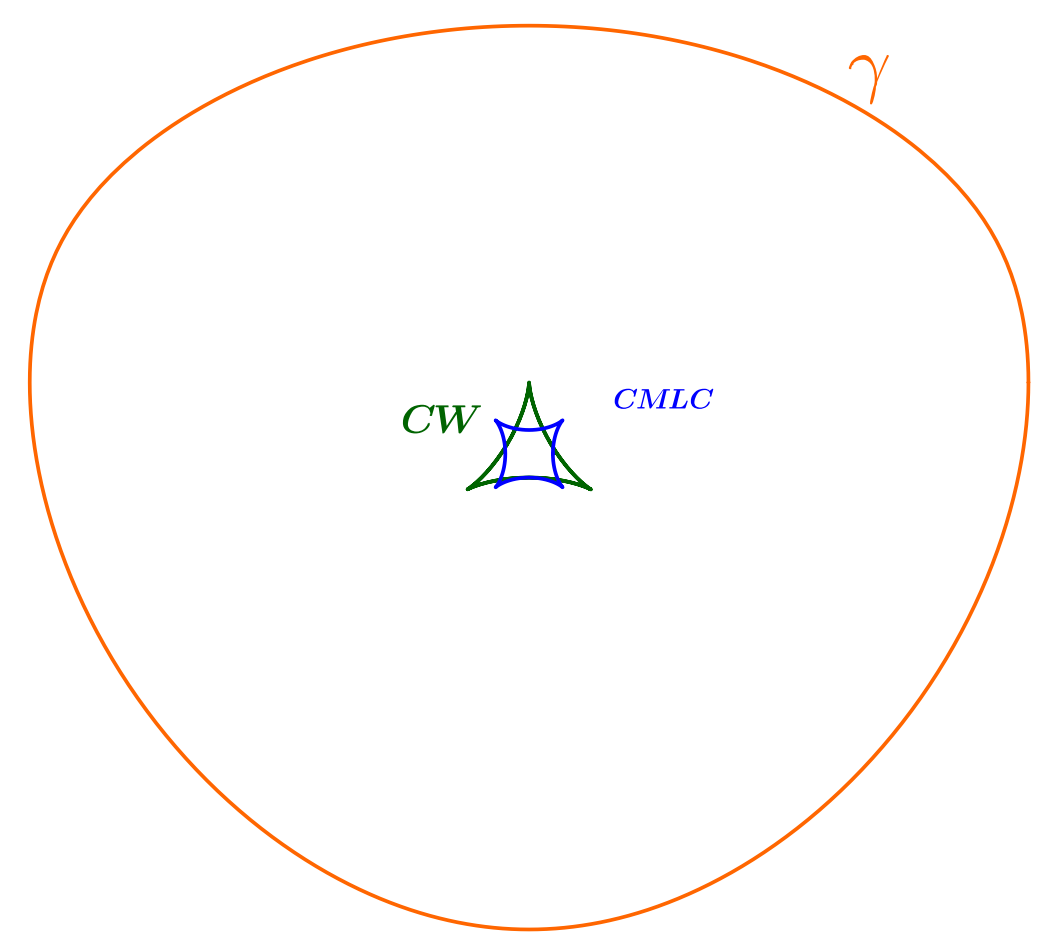

Figura 3.4: Curva $\gamma$, Cáustica $C W(\gamma)$ e $C M L C(\gamma)$ 


\section{4 \\ Igualdade Isoperimétrica em Plano Normado}

Teorema 4.1 (Igualdade Isoperimétrica em Plano Normado). Seja $\gamma \in \mathscr{H}$ no plano $\left(\mathbb{R}^{2}, U\right)$, onde $U$ é a bola unitária. Então

$$
L_{v}^{2}(\gamma)=4 A_{U} \tilde{\mathrm{A}}_{\gamma}-8 A_{U} \tilde{\mathrm{A}}_{C W(\gamma)}-4 A_{U} \tilde{\mathrm{A}}_{C M L C(\gamma)},
$$

onde $L_{v}(\gamma)$ indica o comprimento-v com sinal da curva $\gamma, A_{U}$ indica a área da bola unitária e $\widetilde{\mathrm{A}}_{\gamma}, \widetilde{\mathrm{A}}_{C W(\gamma)}, \widetilde{\mathrm{A}}_{C M L C(\gamma)}$ indicam respectivamente as áreas com sinal das curvas $\gamma, C W(\gamma)$ e $C M L C(\gamma)$.

Demonstração. Pela proposição (3.20) temos

$$
\gamma(\theta)=\left(\frac{\gamma(\theta)+\gamma(\theta+\pi)}{2}\right)+\left(\frac{\gamma(\theta)-\gamma(\theta+\pi)-\bar{w}_{\gamma} u(\theta)}{2}\right)+\frac{\bar{w}_{\gamma}}{2} u(\theta)
$$

isto é,

$$
\gamma(\theta)=C W(\gamma)(\theta)+C M L C(\gamma)(\theta)+\frac{\bar{w}_{\gamma}}{2} u(\theta)
$$

Vamos proceder da seguinte maneira: calcular a área de $\gamma(\theta)$ utilizando a proposição (3.20) e assim obter a Igualdade Isoperimétrica. Iremos também utilizar um resultado de integração por partes

$$
\int_{0}^{2 \pi}\left[f^{\prime}(t), g(t)\right] d t=-\int_{0}^{2 \pi}\left[f(t), g^{\prime}(t)\right] d t
$$

onde $f, g$ são de classe $C^{1}$ e que

$$
\int_{0}^{2 \pi}\left[C M L C(\gamma)(\theta), C W^{\prime}(\gamma)(\theta)\right] d \theta=0
$$




$$
\begin{aligned}
& \widetilde{\mathrm{A}}(\gamma)=\frac{1}{2} \int_{0}^{2 \pi}\left[\gamma(\theta), \gamma^{\prime}(\theta)\right] d \theta \\
& =\frac{1}{2} \int_{0}^{2 \pi}\left[C W(\theta)+C M L C(\theta)+\frac{\bar{w}_{\gamma}}{2} u(\theta), C W^{\prime}(\theta)+C M L C^{\prime}(\theta)+\frac{\bar{w}_{\gamma}}{2} u^{\prime}(\theta)\right] d \theta \\
& =\frac{1}{2} \int_{0}^{2 \pi}\left[C W(\theta), C W^{\prime}(\theta)\right] d \theta+\frac{1}{2} \int_{0}^{2 \pi}\left[C M L C(\theta), C M L C^{\prime}(\theta)\right] d \theta \\
& +\frac{1}{2} \int_{0}^{2 \pi} \frac{\bar{w}_{\gamma}^{2}}{4}\left[u(\theta), u^{\prime}(\theta)\right] d \theta+\frac{1}{2} \int_{0}^{2 \pi}\left[C W(\theta), C M L C^{\prime}(\theta)\right] d \theta \\
& +\frac{1}{2} \int_{0}^{2 \pi} \frac{\bar{w}_{\gamma}}{2}\left[C W(\theta), u^{\prime}(\theta)\right] d \theta+\frac{1}{2} \int_{0}^{2 \pi}\left[C M L C(\theta), C W^{\prime}(\theta)\right] d \theta \\
& +\frac{1}{2} \int_{0}^{2 \pi} \frac{\bar{w}_{\gamma}}{2}\left[C M L C(\theta), u^{\prime}(\theta)\right] d \theta+\frac{1}{2} \int_{0}^{2 \pi} \frac{\bar{w}_{\gamma}}{2}\left[u(\theta), C W^{\prime}(\theta)\right] d \theta \\
& +\frac{1}{2} \int_{0}^{2 \pi} \frac{\bar{w}_{\gamma}}{2}\left[u(\theta), C M L C^{\prime}(\theta)\right] d \theta \\
& =2 \widetilde{\mathrm{A}}_{C W(\gamma)}+\tilde{\mathrm{A}}_{C M L C(\gamma)}+\frac{\bar{w}_{\gamma}^{2}}{4} A_{U}+\frac{1}{2} \int_{0}^{2 \pi}\left[C M L C(\theta), C W^{\prime}(\theta)\right] d \theta \\
& +\frac{1}{2} \int_{0}^{2 \pi}\left[C M L C(\theta), C W^{\prime}(\theta)\right] d \theta+\int_{0}^{2} \frac{\bar{w}_{\gamma}}{2}\left[u(\theta), C W^{\prime}(\theta)\right] d \theta \\
& +\int_{0}^{2} \frac{\bar{w}_{\gamma}}{2}\left[u(\theta), C M L C^{\prime}(\theta)\right] d \theta \\
& =2 \widetilde{\mathrm{A}}_{C W(\gamma)}+\tilde{\mathrm{A}}_{C M L C(\gamma)}+\frac{\bar{w}_{\gamma}^{2}}{4} A_{U}+\frac{\bar{w}_{\gamma}}{2} \int_{0}^{2}\left[u(\theta), C W^{\prime}(\theta)+C M L C^{\prime}(\theta)\right] d \theta \\
& =2 \widetilde{\mathrm{A}}_{C W(\gamma)}+\widetilde{\mathrm{A}}_{C M L C(\gamma)}+\frac{\bar{w}_{\gamma}^{2}}{4} A_{U}+\frac{\bar{w}_{\gamma}}{2} \int_{0}^{2}\left[u(\theta), \gamma^{\prime}(\theta)-\frac{\bar{w}_{\gamma}}{2} u^{\prime}(\theta)\right] d \theta \\
& =2 \tilde{\mathrm{A}}_{C W(\gamma)}+\tilde{\mathrm{A}}_{C M L C(\gamma)}+\frac{\bar{w}_{\gamma}^{2}}{4} A_{U} \\
& +\frac{\bar{w}_{\gamma}}{2}\left[\int_{0}^{2}\left[u(\theta), \gamma^{\prime}(\theta)\right] d \theta-\int_{0}^{2} \frac{\bar{w}_{\gamma}}{2}\left[u(\theta), u^{\prime}(\theta)\right] d \theta\right] \\
& =2 \widetilde{\mathrm{A}}_{C W(\gamma)}+\tilde{\mathrm{A}}_{C M L C(\gamma)}+\frac{\bar{w}_{\gamma}^{2}}{4} A_{U}+\frac{\bar{w}_{\gamma}}{2}\left[L_{V}(\gamma)-\bar{w}_{\gamma} A_{U}\right] \\
& =2 \widetilde{\mathrm{A}}_{C W(\gamma)}+\widetilde{\mathrm{A}}_{C M L C(\gamma)}+\frac{\bar{w}_{\gamma}^{2}}{4} A_{U}+\frac{\bar{w}_{\gamma}}{2}\left[L_{V}(\gamma)-\frac{L_{V}(\gamma)}{A_{U}} A_{U}\right] \\
& =2 \widetilde{\mathrm{A}}_{C W(\gamma)}+\widetilde{\mathrm{A}}_{C M L C(\gamma)}+\frac{\bar{w}_{\gamma}^{2}}{4} A_{U} \\
& =2 \widetilde{\mathrm{A}}_{C W(\gamma)}+\widetilde{\mathrm{A}}_{C M L C(\gamma)}+\frac{L_{V}^{2}(\gamma)}{4 A_{U}} \\
& \Rightarrow L_{V}^{2}(\gamma)=4 A_{U} \widetilde{\mathrm{A}}_{\gamma}-8 A_{U} \widetilde{\mathrm{A}}_{C W(\gamma)}-4 A_{U} \widetilde{\mathrm{A}}_{C M L C(\gamma)}
\end{aligned}
$$


Observe que se $\gamma \in \mathscr{H}$ é uma oval então $\widetilde{\mathrm{A}}_{\gamma}=A_{\gamma}$, isto é, a área com sinal é a própria área limitada pela curva. Dessa forma temos os seguintes corolários.

Corolário 4.2. Seja $\gamma$ oval no plano $\left(\mathbb{R}^{2}, U\right)$, onde $U$ é a bola unitária. Então

$$
L_{V}^{2}(\gamma)=4 A_{U} A_{\gamma}+8 A_{U}\left|\tilde{\mathrm{A}}_{C W(\gamma)}\right|+4 A_{U}\left|\tilde{\mathrm{A}}_{C M L C(\gamma)}\right|
$$

onde $L_{V}(\gamma)$ indica o comprimento da curva $\gamma$ na norma dual, $A_{U}$ e $A_{\gamma}$ indicam respectivamente as áreas da bola unitária e da curva $\gamma, \widetilde{\mathrm{A}}_{C W(\gamma)}$ e $\widetilde{\mathrm{A}}_{C M L C(\gamma)}$ indicam respectivamente as áreas com sinal de $C W(\gamma)$ e $C M L C(\gamma)$.

O próximo corolário é uma generalização do resultado de Zwierzyński em [24].

Corolário 4.3. Seja $\gamma$ oval no plano $\left(\mathbb{R}^{2}, U\right)$, onde $U$ é a bola unitária. Então

$$
L_{V}^{2}(\gamma) \geq 4 A_{U} A_{\gamma}+8 A_{U}\left|\tilde{\mathrm{A}}_{C W(\gamma)}\right|
$$

onde $L_{V}(\gamma)$ indica o comprimento da curva $\gamma$ na norma dual, $A_{U}$ e $A_{\gamma}$ indicam respectivamente as áreas da bola unitária e da curva $\gamma, \widetilde{\mathrm{A}}_{C W(\gamma)}$ indica a área com sinal de $C W$. A igualdade ocorre se, e só se, a curva $\gamma$ tem largura constante.

Corolário 4.4 (Desigualdade Clássica Isoperimétrica no Plano de Minkowski). Seja $\gamma$ oval no plano $\left(\mathbb{R}^{2}, U\right)$, onde $U$ é a bola unitária. Então

$$
L_{V}^{2}(\gamma) \geq 4 A_{U} A_{\gamma}
$$

onde $L_{V}(\gamma)$ indica o comprimento da curva $\gamma$ na norma dual, $A_{U}$ e $A_{\gamma}$ indicam respectivamente as áreas da bola unitária e da curva $\gamma$. A igualdade ocorre se, e só se, a curva $\gamma$ é simétrica e tem largura constante, isto é múltiplo da bola unitária.

Corolário 4.5. Seja $\gamma$ oval no plano $\left(\mathbb{R}^{2}, U\right)$, onde $U$ é a bola unitária. Então

$$
L_{V}^{2}(\gamma) \geq 4 A_{U} A_{\gamma}+4 A_{U}\left|\tilde{\mathrm{A}}_{C M L C(\gamma)}\right|
$$

onde $L_{V}(\gamma)$ indica o comprimento da curva $\gamma$ na norma dual, $A_{U}$ e $A_{\gamma}$ indicam respectivamente as áreas da bola unitária e da curva $\gamma, \widetilde{A}_{C M L C(\gamma)}$ indica a área com sinal de $C M L C(\gamma)$. A igualdade ocorre se, e só se, a curva $\gamma$ é simétrica. 


\section{5}

\section{Ciclóides e Base Ortonormal}

Ciclóides clássicas têm a propriedade de que sua dupla evoluta é homotética a ela mesma. Considerando esta mesma propriedade em um plano normado qualquer, temos a seguinte definição (proposição (3.31)):

Definição 5.1. Definimos como ciclóide as curvas $\omega \in \mathscr{H}$, isto é, fechadas, suaves e parametrizadas por $\theta \in[0,2 \pi]$, cuja função suporte $h_{\omega}(\theta)$ satisfaz a equação

$$
-\frac{1}{\left[u(\theta), u^{\prime}(\theta)\right]}\left(\frac{h_{\omega}^{\prime}(\theta)}{\left[v(\theta), v^{\prime}(\theta)\right]}\right)^{\prime}=\lambda h_{\omega}(\theta), \lambda \in \mathbb{R},
$$

que é a equação diferencial de segunda ordem de Sturm-Liouville exibida em [6].

Todos os resultados obtidos em [6] são baseados na equação de Sturm-Liouville.

Definição 5.2. Dizemos que $\lambda$ é autovalor da equação (5.1) se existe função suporte $h$ periódica de período $2 \pi$ que satisfaz tal equação. A função $h$ é chamada de autovetor.

O conjunto $C^{0}\left(S^{1}\right)$ é o espaço da funções reais contínuas, cujo domínio é a circunferência euclidiana unitária, isto é, $h \in C^{0}\left(S^{1}\right) \Leftrightarrow h: S^{1} \rightarrow \mathbb{R}$. De acordo com [6] vamos munir esse conjunto com produto interno

$$
\left\langle h_{1}, h_{2}\right\rangle=\int_{0}^{2 \pi} h_{1}(\theta) h_{2}(\theta)\left[u(\theta), u^{\prime}(\theta)\right] d \theta
$$

onde $u$ é a fronteira da bola unitária $U$ no plano normado.

Lema 5.3. A transformação linear

$$
T h=-\frac{1}{\left[u, u^{\prime}\right]}\left(\frac{h^{\prime}}{\left[v, v^{\prime}\right]}\right)^{\prime}
$$

é auto-adjunta com respeito ao produto interno (5.2). 


\section{Demonstração.}

$$
\begin{gathered}
\left\langle h_{1}, T h_{2}\right\rangle=\int_{0}^{2 \pi} h_{1} T h_{2}\left[u, u^{\prime}\right] d \theta=-\int_{0}^{2 \pi} h_{1}\left(\frac{h_{2}^{\prime}}{\left[v, v^{\prime}\right]}\right)^{\prime} d \theta=\int_{0}^{2 \pi} \frac{h_{1}^{\prime} h_{2}^{\prime}}{\left[v, v^{\prime}\right]} d \theta \\
=-\int_{0}^{2 \pi} h_{2}\left(\frac{h_{1}^{\prime}}{\left[v, v^{\prime}\right]}\right)^{\prime} d \theta=\int_{0}^{2 \pi} h_{2} T h_{1}\left[u, u^{\prime}\right] d \theta=\left\langle T h_{1}, h_{2}\right\rangle
\end{gathered}
$$

Observe que o núcleo $K$ da transformação $T$ é subespaço das funções constantes. Com efeito

$$
-\frac{1}{\left[u, u^{\prime}\right]}\left(\frac{h^{\prime}}{\left[v, v^{\prime}\right]}\right)^{\prime}=0 \Leftrightarrow\left(\frac{h^{\prime}}{\left[v, v^{\prime}\right]}\right)^{\prime}=0 \Leftrightarrow \frac{h^{\prime}}{\left[v, v^{\prime}\right]}=c(\text { constante }) .
$$

Suponha sem perda de generalidade $c>0$. Assim teremos $h^{\prime}=c\left[v, v^{\prime}\right]>0$. O que implica $h$ crescente, que é um absurdo pois $h$ é periódica e contínua. Portanto $h$ é constante.

Denote agora $L_{0}=K^{\perp}$. Assim temos que para todo $h \mathrm{em} L_{0}$,

$$
\int_{0}^{2 \pi} h(\theta)\left[u, u^{\prime}\right](\theta) d \theta=0
$$

Seja $S: L_{0} \rightarrow C^{0}\left(S^{1}\right)$ a inversa de $T$.

Lema 5.4. Para todo $h \in L_{0}$ seja $g=$ Sh. Então

$$
\|g\|_{\infty} \leq\left\|\left[v, v^{\prime}\right]\right\|_{2}\left\|\left[u, u^{\prime}\right]\right\|_{2}\|h\|_{2}
$$

$e$

$$
\left\|g^{\prime}\right\|_{\infty} \leq\left\|\left[v, v^{\prime}\right]\right\|_{\infty}\left\|\left[u, u^{\prime}\right]\right\|_{2}\|h\|_{2}
$$

Demonstração. Ver [6].

Proposição 5.5. $S:\left(L_{0},\|\cdot \mid\|_{2}\right) \rightarrow\left(L_{0},\|\cdot\|_{2}\right)$ é operador compacto. 
Demonstração. Seja $h_{n}$ sequência limitada em $\left(L_{0},\|\cdot\|_{2}\right)$. Pelo lema (5.3) a sequência $g_{n}=S h_{n}$ é equicontínua e uniformemente limitada. Assim podemos encontrar subsequência $g_{n_{j}}$ convergente na norma $\|\cdot\|_{2}$.

Definição 5.6. Sejam $\left\{h_{k}^{i}\right\},\left\{\lambda_{k}^{i}\right\}, k \in \mathbb{N}, i=1,2$ respectivamente os autovetores e autovalores correspondentes da transformação T lema (5.3).

Utilizando os resultados anteriores temos o seguinte corolário.

Corolário 5.7. O conjunto $\left\{h_{k}^{i}\right\}, k \in \mathbb{N}, i=1,2$, forma uma base ortonormal de $C^{0}\left(S^{1}\right)$.

No caso Euclidiano a base $\left\{h_{k}^{i}\right\}$ é

$$
\{1, \cos (\theta), \sin (\theta), \cos (2 \theta), \sin (2 \theta), \ldots, \cos (k \theta), \sin (k \theta), \ldots\}
$$

chamada de base de Fourier, onde $h_{k}^{1}(\theta)=\cos (k \theta), h_{k}^{2}=\sin (k \theta)$ e $\lambda_{k}^{i}=k^{2}$ e $\lambda_{k}^{i} \geq 1$, ver [22] e [6].

Suponhamos que o raio de curvatura $r(\theta)$ de $\gamma(\theta)$ satisfaça a equação (5.1) com $\lambda \neq 1$, então $h(\theta)=\frac{r(\theta)}{1-\lambda}$ satisfaz (5.1). Reciprocamente se a função suporte $h$ da curva $\gamma$ satisfaz (5.1) então pela equação (2.6), $r(\theta)=h(\theta)(1-\lambda)$ também satisfaz (5.1). Segundo [6], $\lambda=1$ indica as ciclóides abertas que, portanto, não podem ser representadas por funções suporte periódicas. Para $\lambda \neq 1$ a curva $\gamma$ possui tanto para o raio de curvatura como função suporte, na transformação T, mesmos autovalores. Como estamos trabalhando com curvas fechadas fica justificado o uso de $h$ ao invés de $r$ na transformação $T$, Lema (5.3). As cicloides abertas no plano, não serão consideradas, pois não podemos calcular $C W$ e $C M L C$ dessas curvas.

Vamos assumir agora $C^{0}\left(S^{1}\right)$ como conjunto das funções suporte $h$ das curvas $\gamma$, fechadas suaves em $\left(\mathbb{R}^{2},\|\cdot\|_{u}\right)$. Portanto teremos que $K$ representa as curvas que são múltiplos da bola unitária $U$, pois $h(\theta)=[\gamma(\theta), v(\theta)]=$ $[\alpha u(\theta), v(\theta)]=\alpha$ (constante). $L_{0}$ definido pela equação (5.4) representa todas a curvas $\gamma$ que possuem comprimento dual com sinal zero. Portanto dada uma função suporte $h$ em $C^{0}\left(S^{1}\right)$ a menos de uma translação, podemos escrevê-la como segue: 


$$
\begin{gathered}
h=h_{0}+\sum_{k \geq 2} a_{k}^{i} h_{k}^{i} \\
h^{\prime}=\sum_{k \geq 2} a_{k}^{i}\left(h_{k}^{i}\right)^{\prime}
\end{gathered}
$$

onde

$$
\left\langle h_{m}^{i}, h_{n}^{j}\right\rangle=0, s e, m \neq n
$$

e

$$
\left\langle h_{m}^{i}, h_{n}^{j}\right\rangle=A(U), s e, m=n, i=j .
$$

Nesse caso $A(U)$ denota a área da bola unitária $U$ em $\left(\mathbb{R}^{2},\|\cdot\|_{u}\right)$. Por Lemma(4.2) de [6] temos

$$
\begin{gathered}
h_{k}^{i}(\theta)=-h_{k}^{i}(\theta+\pi), \text { kímpar. } \\
h_{k}^{i}(\theta)=h_{k}^{i}(\theta+\pi), k \text { par. }
\end{gathered}
$$

Consequentemente $\left(h_{k}^{i}\right)^{\prime}(\theta)=-\left(h_{k}^{i}\right)^{\prime}(\theta+\pi)$ para $k$ ímpar e $\left(h_{k}^{i}\right)^{\prime}(\theta)=$ $\left(h_{k}^{i}\right)^{\prime}(\theta+\pi)$ para $k$ par.

Teorema 5.8. Seja $\left\{h_{0}, h_{2}^{1}, h_{2}^{2}, h_{3}^{1}, h_{3}^{2}, \ldots, h_{n}^{1}, h_{n}^{2}, \ldots\right\}$ base ortonormal de $C^{0}\left(S^{1}\right), \omega_{k}^{j} \in \mathscr{H}$ ciclóide associada a função suporte $h_{k}^{j}$. Então

1. $C W\left(\omega_{k}^{j}\right)=\omega_{k}^{j}$ e $C M L C\left(\omega_{k}^{j}\right)=\{0\}, k$ impar.

2. $C W\left(\omega_{k}^{j}\right)=\{0\}$ e $C M L C\left(\omega_{k}^{j}\right)=\omega_{k}^{j}, k$ par.

\section{Demostração.}

Para $k$ ímpar a equação (5.9) e a proposição (3.6) implicam que $\omega_{k}^{j}(\theta)=$ $\omega_{k}^{j}(\theta+\pi)$, então $C W\left(\omega_{k}^{j}\right)=\omega_{k}^{j}$. Pela a proposição (3.16) $C M L C\left(\omega_{k}^{j}\right)=\{0\}$. Para $k$ par a equação (5.10) e a proposição (3.9) mostram que $\omega_{k}^{j}$ é simétrica e portanto $C W\left(\omega_{k}^{j}\right)=\{0\}$. Pela equação (5.7) temos 


$$
\begin{aligned}
L_{v}\left(\omega_{k}^{j}\right) & =\int_{0}^{2 \pi} r_{\omega_{k}^{j}}(\theta)\left[u(\theta), u^{\prime}(\theta)\right] d \theta \\
& =\int_{0}^{2 \pi} h_{k_{i}}^{j}\left(1-\lambda_{k}^{j}\right)[u(\theta), u(\theta)] d \theta \\
& =\left(1-\lambda_{k}^{j}\right) \int_{0}^{2 \pi} h_{k_{i}}^{j}[u(\theta), u(\theta)] d \theta=0
\end{aligned}
$$

Portanto $\bar{w}_{\omega_{k}^{j}}=\frac{L_{v}\left(\omega_{k}^{j}\right)}{A(u)}=0$ e simetria implicam $C M L C\left(\omega_{k}^{j}\right)=\omega_{k}^{j}$

Esse resultado indica que podemos utilizar o CW e CMLC como representação geométrica das ciclóides, com $k$ ímpar e $k$ par respectivamente.

\section{1}

\section{Áreas e Comprimentos}

Nessa seção vamos apresentar o cálculo de áreas orientadas e comprimento dual de curvas em $\mathscr{H}$ estudados até o momento, utilizando a base ortonormal de $C^{0}\left(S^{1}\right)$, corolário (5.6), isto é, a série de Fourier generalizada.

$$
\begin{aligned}
L_{V}(\gamma)(\theta) & =\int_{0}^{2 \pi} r(\theta)\left[u(\theta), u^{\prime}(\theta)\right] d \theta=\int_{0}^{2 \pi}\left(h\left[u, u^{\prime}\right]+\left(\frac{h^{\prime}}{\left[v, v^{\prime}\right]}\right)^{\prime}\right)(\theta)\left[u(\theta), u^{\prime}(\theta)\right] d \theta \\
& =\int_{0}^{2 \pi} h(\theta)\left[u, u^{\prime}\right](\theta)+\left(\frac{h^{\prime}(\theta)}{\left[v, v^{\prime}\right](\theta)}\right)^{\prime} d \theta \\
& =\int_{0}^{2 \pi} h(\theta)\left[u, u^{\prime}\right](\theta) d \theta \\
& =\int_{0}^{2 \pi}\left(h_{0}+\sum_{k \geqslant 2} a_{k}^{i} h_{k}^{i}\right)\left[u, u^{\prime}\right](\theta) d \theta \\
& =h_{0} \int_{0}^{2 \pi}\left[u, u^{\prime}\right](\theta) d \theta+\sum_{k \geqslant 2} a_{k}^{i} \int_{0}^{2 \pi} h_{k}^{i}\left[u, u^{\prime}\right](\theta) d \theta \\
& =2 h_{0} \int_{0}^{2 \pi} \frac{1}{2}\left[u, u^{\prime}\right](\theta) d \theta \\
& =2 h_{0} A(U)
\end{aligned}
$$




$$
\begin{aligned}
& \widetilde{\mathrm{A}}(\gamma)=\frac{1}{2} \int_{0}^{2 \pi}\left[\gamma, \gamma^{\prime}\right](\theta) d \theta \\
& =\frac{1}{2} \int_{0}^{2 \pi}\left[h(\theta) u(\theta)+\frac{h^{\prime}(\theta)}{\left[v, v^{\prime}\right](\theta)} v(\theta),\left(h(\theta)\left[u, u^{\prime}\right](\theta)+\left(\frac{h^{\prime}(\theta)}{\left[v, v^{\prime}\right](\theta)}\right)\right)^{\prime} v(\theta)\right] d \theta \\
& =\frac{1}{2} \int_{0}^{2 \pi}\left(h^{2}(\theta)\left[u, u^{\prime}\right](\theta)+h(\theta)\left(\frac{h^{\prime}(\theta)}{\left[v, v^{\prime}\right](\theta)}\right)^{\prime}\right)[u, v](\theta) d \theta \\
& =\frac{1}{2} \int_{0}^{2 \pi}\left(h^{2}(\theta)\left[u, u^{\prime}\right](\theta)+h(\theta)\left(\frac{h^{\prime}(\theta)}{\left[v, v^{\prime}\right](\theta)}\right)^{\prime}\right) d \theta \\
& =\frac{1}{2} \int_{0}^{2 \pi} h^{2}(\theta)\left[u, u^{\prime}\right](\theta)-\frac{\left(h^{\prime}(\theta)\right)^{2}}{\left[v, v^{\prime}\right](\theta)} d \theta \\
& =\frac{1}{2} \int_{0}^{2 \pi}\left(h_{0}+\sum_{\substack{k \geqslant 2 \\
i=1,2}} a_{k}^{i} h_{k}^{i}\right)^{2}\left[u, u^{\prime}\right](\theta)-\frac{1}{\left[v, v^{\prime}\right]}\left(\sum_{\substack{k \geqslant 2 \\
i=1,2}} a_{k}^{i}\left(h_{k}^{i}\right)^{\prime}\right)^{2}(\theta) d \theta \\
& =\frac{1}{2} \int_{0}^{2 \pi}\left(h_{0}^{2}+2 h_{0} \sum_{\substack{k \geqslant 2 \\
i=1,2}} a_{k}^{i} h_{k}^{i}+\left(\sum_{\substack{k \geqslant 2 \\
i=1,2}} a_{k}^{i} h_{k}^{i}\right)^{2}\right)\left[u, u^{\prime}\right](\theta)-\frac{1}{\left[v, v^{\prime}\right]}\left(\sum_{\substack{k \geqslant 2 \\
i=1,2}} a_{k}^{i}\left(h_{k}^{i}\right)^{\prime}\right)^{2}(\theta) d \theta \\
& =\frac{1}{2} h_{0}^{2} \int_{0}^{2 \pi}\left[u, u^{\prime}\right](\theta) d \theta+h_{0} \sum_{\substack{k \geqslant 2 \\
i=1,2}} a_{k}^{i} \int_{0}^{2 \pi} h_{k}^{i}\left[u, u^{\prime}\right](\theta) d \theta+\frac{1}{2} \sum_{\substack{m \geqslant 2 \\
i=1,2}}\left(a_{m}^{i}\right)^{2} \int_{0}^{2 \pi}\left(h_{m}^{i}\right)^{2}\left[u, u^{\prime}\right](\theta) d \theta \\
& +\sum_{\substack{m \neq n \\
i=1,2}} a_{m}^{i} a_{n}^{i} \int_{0}^{2 \pi} h_{m}^{i} h_{n}^{i}\left[u, u^{\prime}\right](\theta) d \theta+\sum_{m \neq n} a_{m}^{1} a_{n}^{2} \int_{0}^{2 \pi} h_{m}^{1} h_{n}^{2}\left[u, u^{\prime}\right](\theta) d \theta \\
& -\frac{1}{2} \sum_{\substack{m \geq 2 \\
i=1,2}}\left(a_{m}^{i}\right)^{2} \int_{0}^{2 \pi} \frac{1}{\left[v, v^{\prime}\right]}\left(\left(h_{m}^{i}\right)^{\prime}\right)^{2}(\theta) d \theta-\sum_{\substack{m \neq n \\
i=1,2}} a_{m}^{i} a_{n}^{i} \int_{0}^{2 \pi} \frac{1}{\left[v, v^{\prime}\right]}\left(h_{m}^{i}\right)^{\prime}\left(h_{n}^{i}\right)^{\prime}(\theta) d \theta \\
& -\sum_{m \neq n} a_{m}^{1} a_{n}^{2} \int_{0}^{2 \pi} \frac{1}{\left[v, v^{\prime}\right]}\left(h_{m}^{1}\right)^{\prime}\left(h_{n}^{2}\right)^{\prime}(\theta) d \theta \\
& =h_{0}^{2} A(U)+\frac{1}{2} \sum_{\substack{k \geq 2 \\
i=1,2}}\left(a_{k}^{i}\right)^{2} \int_{0}^{2 \pi}\left(h_{k}^{i}\right)^{2}\left[u, u^{\prime}\right](\theta)-\frac{1}{\left[v, v^{\prime}\right]}\left(\left(h_{k}^{i}\right)^{\prime}\right)^{2}(\theta) d \theta \\
& =h_{0}^{2} A(U)+\frac{1}{2} \sum_{\substack{k \geq 2 \\
i=1,2}}\left(a_{k}^{i}\right)^{2} \int_{0}^{2 \pi}\left(h_{k}^{i}\right)^{2}\left[u, u^{\prime}\right]\left(\theta+h_{k}^{i}\left(\frac{\left(h_{k}^{i}\right)^{\prime}}{\left[v, v^{\prime}\right]}\right)^{\prime}(\theta) d \theta\right. \\
& =h_{0}^{2} A(U)+\frac{1}{2} \sum_{\substack{k \geq 2 \\
i=1,2}}\left(a_{k}^{i}\right)^{2} \int_{0}^{2 \pi}\left(h_{k}^{i}\right)^{2}\left[u, u^{\prime}\right](\theta)-\left(h_{k}^{i}\right)^{2} \lambda_{k}^{i}\left[u, u^{\prime}\right](\theta) d \theta \\
& =h_{0}^{2} A(U)+\frac{1}{2} \sum_{\substack{k \geq 2 \\
i=1,2}}\left(a_{k}^{i}\right)^{2}\left(1-\lambda_{k}^{i}\right) \int_{0}^{2 \pi}\left(h_{k}^{i}\right)^{2}\left[u, u^{\prime}\right](\theta) d \theta \\
& =h_{0}^{2} A(U)-\frac{A(U)}{2} \sum_{\substack{k \geq 2 \\
i=1,2}}\left(a_{k}^{i}\right)^{2}\left(\lambda_{k}^{i}-1\right) \text {. }
\end{aligned}
$$


Onde $\lambda_{k}^{i}>1$ são autovalores da transformação $T$, lema (5.3).

$$
\begin{aligned}
& 2 \widetilde{\mathrm{A}}_{C W(\gamma)}=\frac{1}{2} \int_{0}^{2 \pi}\left[C W(\gamma), C W^{\prime}(\gamma)\right](\theta) d \theta \\
& =\frac{1}{2} \int_{0}^{2 \pi} h_{C W(\gamma)}^{2}(\theta)\left[u, u^{\prime}\right](\theta)-\frac{\left(h_{C W(\gamma)}^{\prime}(\theta)\right)^{2}}{\left[v, v^{\prime}\right](\theta)} d \theta \\
& =\frac{1}{2} \int_{0}^{2 \pi}\left(\frac{h(\theta)-h(\theta+\pi)}{2}\right)^{2}\left[u(\theta), u^{\prime}(\theta)\right]-\frac{\left(h^{\prime}(\theta)-h^{\prime}(\theta+\pi)\right)^{2}}{4\left[v(\theta), v^{\prime}(\theta)\right]} d \theta \\
& =\frac{1}{2} A(\gamma)-\frac{1}{4} \int_{0}^{2 \pi} h(\theta) h(\theta+\pi)\left[u(\theta), u^{\prime}(\theta)\right]-\frac{h^{\prime}(\theta) h^{\prime}(\theta+\pi)}{\left[v(\theta), v^{\prime}(\theta)\right]} d \theta \\
& =\frac{1}{2} A(\gamma)-\frac{1}{4} \int_{0}^{2 \pi}\left(h_{0}+\sum_{\substack{k \geqslant 2 \\
i=1,2}} a_{k}^{i} h_{k}^{i}(\theta)\right)\left(h_{0}+\sum_{\substack{k \geqslant 2 \\
i=1,2}} a_{k}^{i} h_{k}^{i}(\theta+\pi)\right)\left[u(\theta), u^{\prime}(\theta)\right] \\
& -\frac{1}{\left[v(\theta), v^{\prime}(\theta)\right]}\left(\sum_{\substack{k \geqslant 2 \\
i=1,2}} a_{k}^{i}\left(h_{k}^{i}\right)^{\prime}(\theta)\right)\left(\sum_{\substack{k \geqslant 2 \\
i=1,2}} a_{k}^{i}\left(h_{k}^{i}\right)^{\prime}(\theta+\pi)\right) d \theta \\
& =\frac{1}{2} A(\gamma)-\frac{1}{4} \int_{0}^{2 \pi}\left(h_{0}+\sum_{\substack{k \geqslant 2 \\
i=1,2}} a_{k}^{i} h_{k}^{i}(\theta)\right)\left(h_{0}+\sum_{\substack{k \geqslant 2 \\
i=1,2}} a_{k}^{i}(-1)^{k} h_{k}^{i}(\theta)\right)\left[u(\theta), u^{\prime}(\theta)\right] \\
& -\frac{1}{\left[v(\theta), v^{\prime}(\theta)\right]}\left(\sum_{\substack{k \geqslant 2 \\
i=1,2}} a_{k}^{i}\left(h_{k}^{i}\right)^{\prime}(\theta)\right)\left(\sum_{\substack{k \geqslant 2 \\
i=1,2}} a_{k}^{i}(-1)^{k}\left(h_{k}^{i}\right)^{\prime}(\theta)\right) d \theta \\
& =\frac{1}{2} A(\gamma)-\frac{1}{4}\left(2 h_{0}^{2} A(U)-A(U) \sum_{\substack{k \geqslant 2 \\
i=1,2}}(-1)^{k}\left(a_{k}^{i}\right)^{2}\left(\lambda_{k}^{i}-1\right)\right)
\end{aligned}
$$

Substituindo $A(\gamma)$ teremos:

$$
\begin{aligned}
2 \widetilde{\mathrm{A}}_{C W} & =\frac{1}{2}\left(h_{0}^{2} A(U)-\frac{A(U)}{2} \sum_{\substack{k \geqslant 2 \\
i=1,2}}\left(a_{k}^{i}\right)^{2}\left(\lambda_{k}^{i}-1\right)\right)-\frac{1}{2} h_{0}^{2} A(U) \\
& +\frac{A(U)}{4} \sum_{\substack{k \geqslant 2 \\
i=1,2}}(-1)^{k}\left(a_{k}^{i}\right)^{2}\left(\lambda_{k}^{i}-1\right) \\
& =-\frac{A(U)}{2} \sum_{\substack{k=\text { impar } \\
i=1,2}}\left(a_{k}^{i}\right)^{2}\left(\lambda_{k}^{i}-1\right)
\end{aligned}
$$

Agora pela definição (3.7) temos $\bar{w}_{\gamma}=\frac{L_{V}(\gamma)}{A(U)}=\frac{2 h_{0} A(U)}{A(U)}=2 h_{0}$. 


$$
\begin{aligned}
& \widetilde{\mathrm{A}}_{C M L C(\gamma)}=\frac{1}{8} \int_{0}^{2 \pi}\left[C M L C(\gamma)(\theta),(C M L C)^{\prime}(\gamma)(\theta)\right] d \theta \\
& =\frac{1}{8} \int_{0}^{2 \pi} h_{C M L C(\gamma)(\theta)}^{2}\left[u(\theta), u^{\prime}(\theta)\right]-\frac{\left(h_{C M L C(\gamma)(\theta)}^{\prime}\right)^{2}}{\left[v(\theta), v^{\prime}(\theta)\right]} d \theta \\
& =\frac{1}{8} \int_{0}^{2 \pi}\left(h(\theta)+h(\theta+\pi)-\bar{w}_{\gamma}\right)^{2}\left[u(\theta), u^{\prime}(\theta)\right]-\frac{\left(h^{\prime}(\theta)+h^{\prime}(\theta+\pi)\right)^{2}}{\left[v(\theta), v^{\prime}(\theta)\right]} d \theta \\
& =\frac{1}{8} \int_{0}^{2 \pi}\left(h^{2}(\theta)+2 h(\theta) h(\theta+\pi)+h^{2}(\theta+\pi)-2 h(\theta) \bar{w}_{\gamma}-2 h(\theta+\pi) \bar{w}_{\gamma}\right)\left[u, u^{\prime}\right](\theta) \\
& +\left(\bar{w}_{\gamma}\right)^{2}\left[u, u^{\prime}\right](\theta)-\frac{\left(\left(h^{\prime}(\theta)\right)^{2}+2 h^{\prime}(\theta) h^{\prime}(\theta+\pi)+\left(h^{\prime}(\theta+\pi)\right)^{2}\right.}{\left[v(\theta), v^{\prime}(\theta)\right]} d \theta \\
& =\frac{1}{4}\left(2 A(\gamma)-4 \bar{w}_{\gamma} h_{0} A(U)+\left(\bar{w}_{\gamma}\right)^{2} A(U)\right) \\
& +\frac{1}{4} \int_{0}^{2 \pi} h(\theta) h(\theta+\pi)\left[u(\theta), u^{\prime}(\theta)\right]-\frac{h^{\prime}(\theta) h^{\prime}(\theta+\pi)}{\left[v(\theta), v^{\prime}(\theta)\right]} d \theta \\
& =\frac{1}{4}\left(2 A(\gamma)-4 h_{0}^{2} A(U)+\left(2 h_{0}^{2} A(U)-A(U) \sum_{\substack{k \geqslant 2 \\
i=1,2}}\left(a_{k}^{i}\right)^{2}(-1)^{k}\left(\lambda_{k}^{i}-1\right)\right)\right) \\
& =\frac{1}{4}\left(2 A(\gamma)-2 h_{0}^{2} A(U)-A(U) \sum_{\substack{k \geqslant 2 \\
i=1,2}}\left(a_{k}^{i}\right)^{2}(-1)^{k}\left(\lambda_{k}^{i}-1\right)\right) .
\end{aligned}
$$

Substituindo $A(\gamma)$ teremos:

$$
\tilde{\mathrm{A}}_{C M L C(\gamma)}=-\frac{1}{2}\left(A(U) \sum_{\substack{k=p a r \\ i=1,2}}\left(a_{k}^{i}\right)^{2}\left(\lambda_{k}^{i}-1\right)\right), \lambda_{k}^{i}>1 .
$$

Corolário 5.9. Toda curva $\gamma \in \mathscr{H}$ com $v$-comprimento zero possui $\widetilde{\mathrm{A}}(\gamma)<0$. Em particular a proposição (3.26) está demonstrada.

\section{2}

\section{Outra prova - Igualdade Isoperimétrica em Plano Normado}

Teorema 5.10 (Igualdade Isoperimétrica em Plano Normado). Seja $\gamma \in \mathscr{H}$ no plano $\left(\mathbb{R}^{2}, U\right)$, onde $U$ é a bola unitária. Então

$$
L_{v}^{2}(\gamma)=4 A_{U} \tilde{\mathrm{A}}_{\gamma}-8 A_{U} \widetilde{\mathrm{A}}_{C W(\gamma)}-4 A_{U} \widetilde{\mathrm{A}}_{C M L C(\gamma)}
$$

onde $L_{V}(\gamma)$ indica o comprimento-v com sinal da curva $\gamma, A_{U}$ indica a área 
da bola unitária e $\widetilde{\mathrm{A}}_{\gamma}, \widetilde{\mathrm{A}}_{C W(\gamma)}, \widetilde{\mathrm{A}}_{C M L C(\gamma)}$ indicam respectivamente as áreas com sinal das curvas $\gamma, C W(\gamma)$ e $C M L C(\gamma)$.

\section{Demonstração.}

Considere $\gamma$ oval parametrizada por $\theta$. Com o uso da base ortonormal de $C^{0}\left(S^{1}\right)$ sabemos que:

$$
\begin{gathered}
L_{v}(\gamma)(\theta)=2 h_{0} A(U), \\
\widetilde{\mathrm{A}}(\gamma)=h_{0}^{2} A_{U}-\frac{A_{U}}{2} \sum_{\substack{k \geq 2 \\
i=1,2}}\left(a_{k}^{i}\right)^{2}\left(\lambda_{k}^{i}-1\right), \\
\widetilde{\mathrm{A}}_{C W(\gamma)}=-\frac{A_{U}}{4} \sum_{\substack{k=i m p a r \\
i=1,2}}\left(a_{k}^{i}\right)^{2}\left(\lambda_{k}^{i}-1\right), \\
\tilde{\mathrm{A}}_{C W M S(\gamma)}=-\frac{A_{U}}{2} \sum_{\substack{k=p a r \\
i=1,2}}\left(a_{k}^{i}\right)^{2}\left(\lambda_{k}^{i}-1\right) .
\end{gathered}
$$

Observe que $\lambda_{k}^{i}>1(\operatorname{ver}[6]), \tilde{\mathrm{A}}_{C W(\gamma)} \leq 0$ e $\tilde{\mathrm{A}}_{C W M S(\gamma)} \leq 0$. Daí temos:

$$
\begin{aligned}
& 4 A_{U} A_{\gamma}-8 A_{U} \tilde{\mathrm{A}}_{C W(\gamma)}-4 A_{U} \tilde{\mathrm{A}}_{C W M S(\gamma)}=4 A_{U}\left(h_{0}^{2} A_{U}-\frac{A_{U}}{2} \sum_{\substack{k \geq 2 \\
i=1,2}}\left(a_{k}^{i}\right)^{2}\left(\lambda_{k}^{i}-1\right)\right) \\
& +8 A_{U} \frac{A_{U}}{4} \sum_{\substack{k=1 \text { mpar } \\
i=1,2}}\left(a_{k}^{i}\right)^{2}\left(\lambda_{k}^{i}-1\right)+4 A_{U} \frac{A_{U}}{2} \sum_{\substack{k=\text { par } \\
i=1,2}}\left(a_{k}^{i}\right)^{2}\left(\lambda_{k}^{i}-1\right)=4 h_{0}^{2}\left(A_{U}\right)^{2}=L_{V}^{2}(\gamma)
\end{aligned}
$$




\section{6 \\ Circulo unitário e dual discretos, Função Suporte e Raio de Curvatura}

Vamos utilizar agora como bola unitária de Minkowski o polígono convexo e fechado $U=\left\{U_{1}, \ldots, U_{2 n}\right\}$ cujos vértices $U_{i}$ estão dispostos em sentido anti-horário, $1 \leq i \leq n$ e $n, i \in \mathbb{N}$. Por definição o conjunto convexo $U$ é simétrico e portanto $U_{i+n}=-U_{i}$. Iremos considerar também que $\left[U_{i}, U_{i+1}-U_{i}\right] \geq 0$. Por consequência da simetria $U$ possui lados opostos paralelos, isto é, $U_{i} U_{i+1} \| U_{i+n} U_{i+n+1}$.

Para uma lista de números, ou vetores $\left\{L_{i}\right\}_{i \in \mathbb{N}}$, nós definimos operadores diferença $\Delta$ tal que $\Delta_{i} L=L_{i+1}-L_{i}$ e $\nabla$ tal que $\nabla_{i}=\Delta_{i-1}$.

De modo análogo ao caso contínuo, a bola unitária dual $U^{*}$ pode ser identificada, segundo [5] e [7], como único polígono $V=\left\{V_{1}, \ldots, V_{2 n}\right\}$ do plano que satisfaz

$$
\begin{gathered}
{\left[U_{i}, V_{i}\right]=1} \\
{\left[\Delta_{i} U, V_{i}\right]=\left[U_{i+1}, \Delta_{i} V\right]=0 .}
\end{gathered}
$$

Nessas condições definimos

$$
\begin{aligned}
V_{i} & =\frac{1}{\left[U_{i}, U_{i+1}\right]} \Delta_{i} U \\
U_{i+1} & =-\frac{1}{\left[V_{i}, V_{i+1}\right]} \Delta_{i} V .
\end{aligned}
$$

Podemos provar que $V$ é simétrico, convexo e tem mesma orientação que $U$. Uma prova que o polígono $\mathrm{V}$ é a bola unitária dual pode ser visto em [5][p.6].

Definição 6.1. Definimos o conjunto $C_{U}$ como espaço dos polígonos $P=$ $\left\{P_{1}, \ldots, P_{2 n}\right\}$, fechados, cujos lados são paralelos aos lados correspondentes da bola unitária $U=\left\{U_{1}, \ldots, U_{2 n}\right\}$, isto é, $P_{i} P_{i+1} \| U_{i} U_{i+1}$.

Definição 6.2 (Rosáceas discretas). Definimos o conjunto $C_{U}-m$, m inteiro positivo, como espaço dos polígonos

$$
P=\left\{P_{1}, \ldots, P_{2 n}, P_{2 n+1}, \ldots, P_{4 n}, \ldots, P_{2 n(m-1)+1}, \ldots, P_{2 m n}\right\}
$$


fechados, cujo número de rotações é $m$ e para cada $j \in\{1, \ldots, 2 m n\}$ temos $P_{j} P_{j+1} \| U_{i} U_{i+1}$ onde $i \equiv j \bmod (2 n)$ e $i \in\{1, \ldots, 2 n\}$.

Definição 6.3. Definimos a função suporte $h$ de $P \in C_{U}$ como a $2 n-$ upla $h=\left(h_{1}, h_{2}, \ldots, h_{2 n}\right)$ onde

$$
h_{i}=\left[P_{i}, V_{i}\right]=\left[P_{i+1}, V_{i}\right]
$$

Dessa forma verificamos que $\left[P_{i}, V_{i}\right]=h_{i}$ e $\left[P_{i}, U_{i}\right]=-\frac{\Delta_{i-1} h}{\left[V_{i-1}, V_{i}\right]}$. Portanto para todo polígono $P$ em $C_{U}$ podemos escrever

$$
P_{i}=h_{i} U_{i}+\left(\Delta_{i-1} h\right) \frac{V_{i}}{\left[V_{i-1}, V_{i}\right]}
$$

A partir da equação 6.4 podemos calcular $\Delta_{i} P$ em função de $h_{i}$

$$
\begin{aligned}
\Delta_{i} P & =P_{i+1}-P_{i} \\
& =h_{i+1} U_{i+1}+\left(\Delta_{i} h\right) \frac{V_{i+1}}{\left[V_{i}, V_{i+1}\right]}-h_{i} U_{i}-\left(\Delta_{i-1} h\right) \frac{V_{i}}{\left[V_{i-1}, V_{i}\right]} \\
& =h_{i+1}\left(-\frac{V_{i+1}-V_{i}}{\left[V_{i}, V_{i+1}\right]}\right)+\frac{\left(h_{i+1}-h_{i}\right) V_{i+1}}{\left[V_{i}, V_{i+1}\right]}+h_{i} \frac{\left(V_{i}-V_{i-1}\right)}{\left[V_{i-1}, V_{i}\right]}-\frac{\left(h_{i}-h_{i-1}\right) V_{i}}{\left[V_{i-1}, V_{i}\right]} \\
& =h_{i}\left(U_{i+1}-U_{i}\right)+V_{i}\left(\frac{h_{i+1}-h_{i}}{\left[V_{i}, V_{i+1}\right]}-\frac{h_{i}-h_{i-1}}{\left[V_{i-1}, V_{i}\right]}\right) \\
& =h_{i} V_{i}\left[U_{i}, U_{i+1}\right]+V_{i}\left(\frac{\Delta_{i} h}{\left[V_{i}, V_{i+1}\right]}-\frac{\Delta_{i-1} h}{\left[V_{i-1}, V_{i}\right]}\right) \\
& =\left(h_{i}+\frac{1}{\left[U_{i}, U_{i+1}\right]} \nabla_{i}\left(\frac{\Delta_{i} h}{\left[V_{i}, V_{i+1}\right]}\right)\right)\left(U_{i+1}-U_{i}\right)
\end{aligned}
$$

Assim temos a equação

$$
\Delta_{i} P=\left(h_{i}+\frac{1}{\left[U_{i}, U_{i+1}\right]} \nabla_{i}\left(\frac{\Delta_{i} h}{\left[V_{i}, V_{i+1}\right]}\right)\right) \Delta_{i} U .
$$

Definição 6.4. Definimos o raio de curvatura $r$ do polígono $P$ em $C_{U}$ como sendo a sequência real $\left(r_{i}\right)_{i \in \mathbb{N}}$, tal que

$$
\Delta_{i} P=r_{i} \cdot \Delta_{i} U
$$

Importante destacar que o raio de curvatura $r_{i}$ está associado ao segmento $P_{i} P_{i+1}, r_{i+2 n}=r_{i}$ e que dado um polígono $P$ em $C_{U}$ podemos representá-lo pelo vetor $r=\left(r_{i}\right)_{i=1,2 \ldots, 2 n}$. Podemos também escrever o raio de curvatura em 
Capítulo 6. Circulo unitário e dual discretos, Função Suporte e Raio de Curvatura

termos da função suporte como segue.

$$
r_{i}=h_{i}+\frac{1}{\left[U_{i}, U_{i+1}\right]} \nabla_{i}\left(\frac{\Delta_{i} h}{\left[V_{i}, V_{i+1}\right]}\right)
$$

\section{1}

\section{Exemplos}

Exemplo 6.5. Podemos calcular o dual de $U$ a partir da equação (6.1). Ver figura 6.1.

Como $U_{1}=\left(\frac{\sqrt{3}}{2}, \frac{1}{2}\right), U_{2}=(0,1), U_{3}=\left(-\frac{\sqrt{3}}{2}, \frac{1}{2}\right), U_{4}=\left(-\frac{\sqrt{3}}{2},-\frac{1}{2}\right), U_{5}=$ $(0,-1), U_{6}=\left(\frac{\sqrt{3}}{2},-\frac{1}{2}\right)$ teremos $V_{1}=\left(-1, \frac{\sqrt{3}}{3}\right), V_{2}=\left(-1,-\frac{\sqrt{3}}{3}\right), V_{3}=$ $\left(0, \frac{-2 \sqrt{3}}{3}\right), V_{4}=\left(1,-\frac{\sqrt{3}}{3}\right), V_{5}=\left(1, \frac{\sqrt{3}}{3}\right), V_{6}=\left(0, \frac{2 \sqrt{3}}{3}\right)$ ou basta observar que $V_{i}=\frac{2 \sqrt{3}}{3} \Delta_{i} U$, para todo $i$. Ou seja, $V$ é um hexágono regular tal que $\left\|V_{i}\right\|_{u}=\frac{2 \sqrt{3}}{3}$. Nesse caso o raio de curvatura de $V$ é $r=\left(\frac{2 \sqrt{3}}{3}, \frac{2 \sqrt{3}}{3}, \ldots, \frac{2 \sqrt{3}}{3}\right)$.

Exemplo 6.6. Considere como bola unitária $U$ o hexágono descrito no exemplo (2.3). Seja $P \in C_{U}$ com raio de curvatura $r=\left(\frac{1}{2}, 1, \frac{1}{2}, \frac{3}{4}, \frac{3}{4}, \frac{3}{4}\right)$. Ver 6.2.

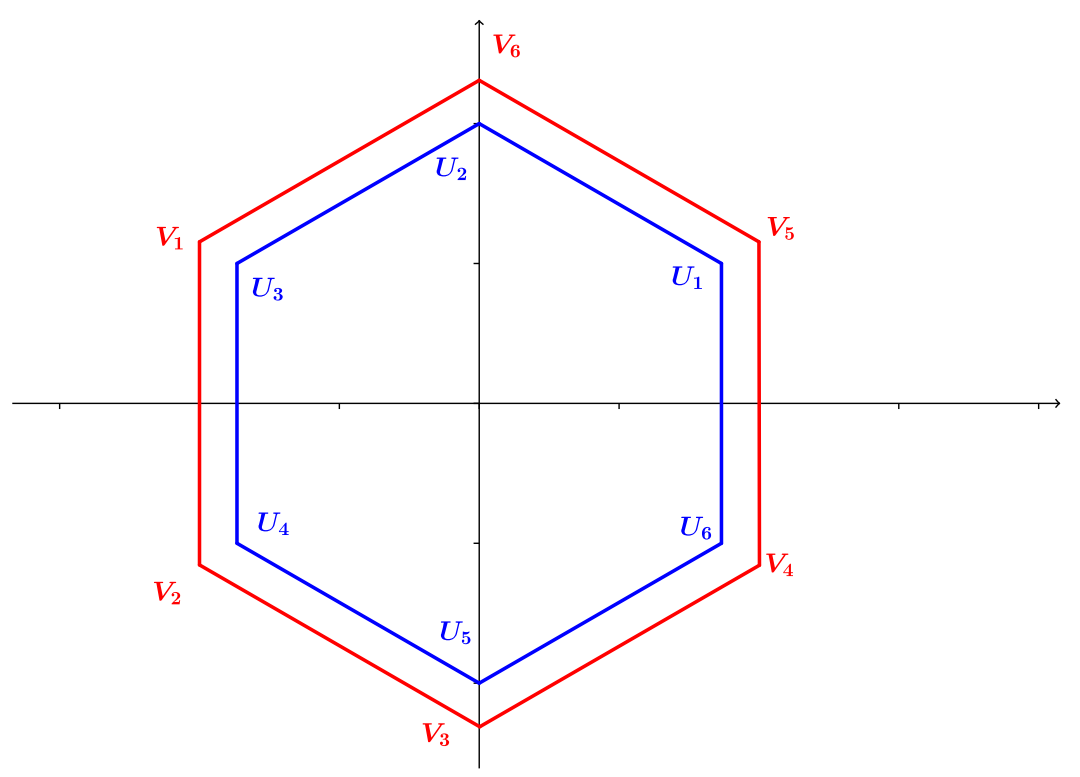

Figura 6.1: Bola unitária $U$ e seu dual $V$ 
Capítulo 6. Circulo unitário e dual discretos, Função Suporte e Raio de Curvatura

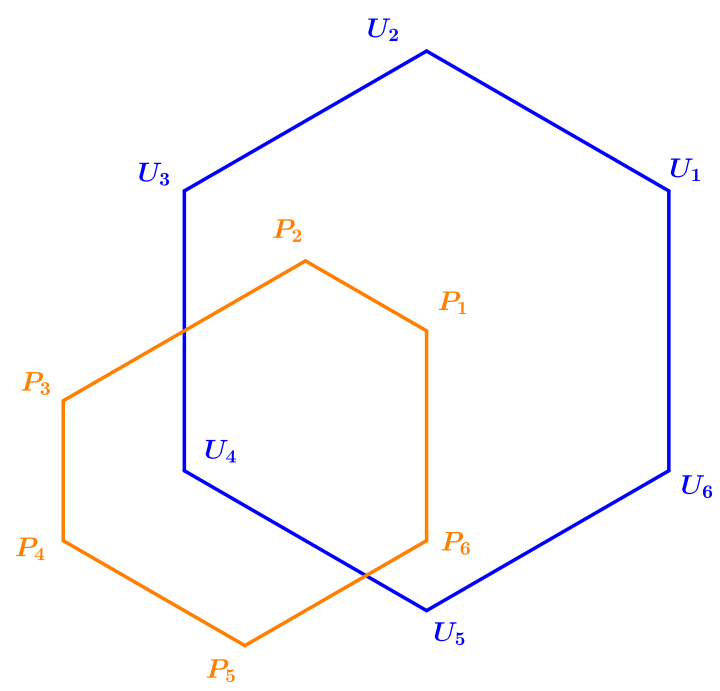

Figura 6.2: Polígono $P \in C_{U}$ onde $r=\left(\frac{1}{2}, 1, \frac{1}{2}, \frac{3}{4}, \frac{3}{4}, \frac{3}{4}\right)$ e bola unitária 


\section{7}

\section{Cáustica de Wigner e Conjunto de Medida de Largura Constante-Discreto}

Considere o polígono $P=\left\{P_{1}, \ldots, P_{2 n}\right\}$ em $C_{U}$, isto é, fechado e $\Delta_{i} P=$ $r_{i} \Delta_{i} U$ onde $U$ é bola unitária do plano normado.

Definição 7.1 (Paralelos). Chamamos de c-paralelo discreto de $P$ o polígono: $P(c)=\left\{P_{1}(c), \ldots, P_{i}(c), \ldots, P_{2 n}(c)\right\}$ onde $i \in\{1,2 \ldots, 2 n\} e$

$$
P_{i}(c)=\left\{P_{i}+c U_{i} ; c \in \mathbb{R}\right\}
$$

Como consequência da definição teremos que $P(c)$ é fechado, $P(0)=P$ e a convexidade depende do valor de $c$. A função suporte do paralelo é

$$
h_{P_{i}(c)}=\left[P_{i}(c), V_{i}\right]=\left[P_{i}+c U_{i}, V_{i}\right]=h_{i}+c
$$

e seu raio de curvatura dado por $r_{i}+c$ pois $\Delta_{i} P(c)=\left(r_{i}+c\right) \Delta_{i} U$.

Definição 7.2 (Comprimento- $V$ com sinal). Seja $P$ polígono em $C_{U}$. Lembrando que $\Delta_{i} P=r_{i}\left[U_{i}, U_{i+1}\right] V_{i}$, definimos o comprimento-V com sinal como

$$
L_{V}(P)=\sum_{i=1}^{2 n} r_{i}\left[U_{i}, U_{i+1}\right]
$$

Nesse caso podemos ter comprimento $-V$ com sinal igual a zero.

Definição 7.3 (Área com sinal). Seja $P$ polígono em $C_{U}$. Definimos a área com sinal de $P$ como

$$
\widetilde{\mathrm{A}}(P)=\frac{1}{2} \sum_{i=1}^{2 n}\left[P_{i}, \Delta_{i} P\right]
$$

Podemos ter, com essa definição, área com sinal assumindo valor negativo. Vale destacar que se $P \in C_{U}$ é polígono convexo, a área com sinal passa a ser a própria área limitada pelo polígono. 
Capítulo 7. Cáustica de Wigner e Conjunto de Medida de Largura

\section{1}

\section{Cáustica de Wigner e Curvas de Largura Constante}

Definição 7.4. Chamamos Evoluta de Área discreta ou Cáustica de Wigner discreta do polígono $P \in C_{U}$ o conjunto:

$$
C W\left(P_{i}\right)=\frac{P_{i}+P_{i+n}}{2}
$$

Como $C W\left(P_{i}\right)=C W\left(P_{i+n}\right)$ temos que $C W\left(P_{i}\right)$ dá duas voltas quando $i$ varia de 1 até $2 n$.

A função suporte de $C W\left(P_{i}\right)$ é

$$
h_{C W\left(P_{i}\right)}=\left[C W\left(P_{i}\right), V_{i}\right]=\left[\frac{P_{i}+P_{i+n}}{2}, V_{i}\right]=\frac{h_{i}}{2}-\frac{h_{i+n}}{2} .
$$

Definição 7.5 (Largura Constante). Dizemos que o polígono $P \in C_{U}$ possui largura constante se, $h_{i}+h_{i+n}=2 d, d>0(d$ constante $)$. Nesse caso dizemos que a largura do Polígono $P$ é $2 d$.

Na prática o polígono tem largura constante se a distância, na $U$-norma, entre os lados opostos paralelos é constante(2d).

Proposição 7.6. $P \in C_{U}$ tem largura constante $2 d \Leftrightarrow P_{i}-P_{i+n}=2 d U_{i}, d$ constante.

Demonstração. $(\Rightarrow)$ Da equação (6.4) temos

$$
\begin{gathered}
P_{i}=h_{i} U_{i}+\left(\Delta_{i-1} h\right) \frac{V_{i}}{\left[V_{i-1}, V_{i}\right]} \\
P_{i}-P_{i+n}=\left(h_{i} U_{i}-h_{i+n} U_{i+n}\right)+\frac{h_{i}+h_{i+n}-\left(h_{i-1}+h_{i-1+n}\right)}{\left[V_{i-1}, V_{i}\right]} V_{i} \\
P_{i}-P_{i+n}=\left(h_{i} U_{i}+h_{i+n} U_{i}\right)=\left(h_{i}+h_{i+n}\right) U_{i}=2 d U_{i}
\end{gathered}
$$

$(\Leftarrow)$

$$
\begin{aligned}
h_{i}+h_{i+n} & =\left[P_{i}, V_{i}\right]+\left[P_{i+n}, V_{i+n}\right] \\
& =\left[P_{i}-P_{i+n}, V_{i}\right] \\
& =\left[2 d U_{i}, V_{i}\right] \\
& =2 d(\text { constante })
\end{aligned}
$$


Definição 7.7 (Largura Média). Chamamos de largura média de um polígono $P$ em $C_{U}$ o número $\bar{w}_{P}=\frac{L_{V}(P)}{A(U)}$, onde $L_{V}(P)$ é o comprimento- $V$ com sinal do polígono $P$ na norma dual e $A(U)$ é a área da bola unitária $U$.

Teorema 7.8 (Barbier-Discreto). Seja $P \in C_{U}$ com largura constante $2 d$ então $L_{V}(\gamma)=2 d A(U)$, onde $U$ é a bola unitária.

\section{Demonstração.}

$$
\begin{aligned}
L_{V}(P) & =\sum_{i=1}^{2 n} r_{i}\left[U_{i}, \Delta_{i} U\right]=\sum_{i=1}^{2 n}\left[U_{i}, \Delta_{i} P\right] \\
& =\sum_{i=1}^{2 n}\left[P_{i}, \Delta_{i} U\right]=\frac{1}{2} \sum_{i=1}^{2 n}\left[P_{i}, \Delta_{i} U\right]+\left[P_{i+n}, \Delta_{i+n} U\right] \\
& =\frac{1}{2} \sum_{i=1}^{2 n}\left(h_{i}+h_{i+n}\right)\left[U_{i}, U_{i+1}\right] \\
& =2 d \frac{1}{2} \sum_{i=1}^{2 n}\left[U_{i}, U_{i+1}\right]=2 d A(U)
\end{aligned}
$$

Caso o polígono $P$ possua largura constante $2 d$ então pelo Teorema de Barbier discreto $L_{V}(P)=A(U) 2 d($ ver [5]). Nesse caso a largura média e a largura da curva são iguais.

Proposição 7.9. A Cáustica de Wigner é um c-paralelo de $P \in C_{U} \Leftrightarrow P$ tem largura constante.

Demonstração. $(\Rightarrow)$

$$
\frac{P_{i}+P_{i+n}}{2}=P_{i}+c U_{i} \Rightarrow P_{i}-P_{i+n}=-2 c U_{i}
$$

Pela proposição (8.4) o polígono $P$ tem largura constante.

$$
\begin{aligned}
(\Leftarrow) \quad & \\
C W\left(P_{i}\right) & =\frac{P_{i}+P_{i+n}}{2} \\
& =P_{i}-\frac{P_{i}-P_{i+n}}{2} \\
& =P_{i}-\frac{c}{2} U_{i}
\end{aligned}
$$


Capítulo 7. Cáustica de Wigner e Conjunto de Medida de Largura Constante-Discreto

Proposição 7.10. $P \in C_{U}, C W(P)=\{0\} \Leftrightarrow P$ é simétrico.

Demonstração. $C W\left(P_{i}\right)=\frac{P_{i}+P_{i+n}}{2}=\frac{P_{i}-P_{i}}{2}=0$

Proposição 7.11. $P \in C_{U}, C W(C W(P))=C W(P)$

Demonstração.

$$
\begin{aligned}
C W\left(C W\left(P_{i}\right)\right) & =\frac{C W\left(P_{i}\right)+C W\left(P_{i+n}\right)}{2} \\
& =\frac{C W\left(P_{i}+C W\left(P_{i}\right)\right.}{2} \\
& =\frac{2 C W\left(P_{i}\right)}{2} \\
& =C W\left(P_{i}\right)
\end{aligned}
$$

Proposição 7.12. $P \in C_{U}, C W(P(c))=C W(P)$

Demonstração.

$$
\begin{aligned}
C W\left(P_{i}(c)\right) & =\frac{P_{i}+c U_{i}+P_{i+n}+c U_{i+n}}{2} \\
& =\frac{P_{i}+P_{i+n}}{2} \\
& =C W\left(P_{i}\right)
\end{aligned}
$$

Proposição 7.13. $P \in C_{U}, C W(P)$ tem comprimento-v zero.

Demonstração.

$$
\begin{aligned}
L_{V}(C W(P)) & =\sum_{i=1}^{2 n} \frac{r_{i}-r_{i+n}}{2}\left[U_{i}, U_{i+1}\right] \\
& =\frac{1}{2}\left[\sum_{i=1}^{2 n} r_{i}\left[U_{i}, U_{i+1}\right]-\sum_{i=1}^{2 n} r_{i+n}\left[U_{i}, U_{i+1}\right]\right] \\
& =\frac{1}{2}\left[\sum_{i=1}^{2 n} r_{i}\left[U_{i}, U_{i+1}\right]-\sum_{i=1}^{2 n} r_{i+n}\left[U_{i+n}, U_{i+1+n}\right]\right] \\
& =\frac{1}{2}\left[L_{V}(P)-L_{V}(P)\right]=0
\end{aligned}
$$


Capítulo 7. Cáustica de Wigner e Conjunto de Medida de Largura Constante-Discreto

\section{2}

\section{Conjunto de Medida de Largura Constante e Curvas Simétricas}

Definição 7.14 (CMLC). Considere o polígono $P$ em $C_{U}$. Definimos o Conjunto de Medida de Largura Constante, abreviado por CMLC, discreto como

$$
C M L C(P)=\left\{\frac{P_{i}-P_{i+n}-\bar{w}_{P} U_{i}}{2}, i=1, \ldots, 2 n\right\}
$$

O polígono $C M L C(P)$ está bem definido pois é fechado e $\Delta_{i}\left(C M L C\left(P_{i}\right)\right)$ é paralelo a $\Delta_{i} U$.

A função suporte do CMLC e o raio de curvatura são respectivamente:

$$
\begin{gathered}
h_{C M L C(P)}=\left[\frac{P_{i}-P_{i+n}-\bar{w}_{P} U_{i}}{2}, V_{i}\right]=\frac{1}{2}\left(h_{i}+h_{i+n}-\bar{w}_{P}\right) \\
\Delta_{i} C M L C(P)=\frac{1}{2}\left\{\Delta_{i} P-\Delta_{i+n} P-\bar{w}_{P} \Delta_{i} U\right\}=\frac{r_{i}+r_{i+n}-\bar{w}_{P}}{2} \Delta_{i} U \\
r_{C M L C(P)}=\frac{r_{i}+r_{i+n}-\bar{w}_{P}}{2}
\end{gathered}
$$

Proposição 7.15. $P \in C_{U}, C M L C(P)$ é simétrico.

Demonstração.

$$
\begin{aligned}
C M L C\left(P_{i+n}\right) & =\frac{P_{i+n}-P_{i+2 n}-\bar{w}_{P} U_{i+n}}{2} \\
& =\frac{P_{i+n}-P_{i}+\bar{w}_{P} U_{i}}{2} \\
& =-\frac{P_{i}-P_{i+n}-\bar{w}_{P} U_{i}}{2} \\
& =-C M L C\left(P_{i}\right)
\end{aligned}
$$

Proposição 7.16. $C M L C(P)$ é paralelo de $P \in C_{U} \Leftrightarrow P$ é simétrico.

Demonstração. $(\Rightarrow)$ 
Capítulo 7. Cáustica de Wigner e Conjunto de Medida de Largura

$$
\begin{aligned}
C M L C\left(P_{i}\right) & =P_{i}+c U_{i} \Rightarrow \\
P_{i} & =C M L C\left(P_{i}\right)-c U_{i} \\
P_{i+n} & =C M L C\left(P_{i+n}\right)-c U_{i+n}=-P_{i}
\end{aligned}
$$

$(\Leftarrow)$

$$
\begin{aligned}
C M L C\left(P_{i}\right) & =\frac{P_{i}-P_{i+n}-\bar{w}_{P} U_{i}}{2} \\
& =\frac{P_{i}+P_{i}-\bar{w}_{P} U_{i}}{2} \\
& =P_{i}-\frac{\bar{w}_{P}}{2} U_{i}
\end{aligned}
$$

Teorema 7.17. Considere o polígono $P$ em $C_{U}$. CMLC $(P)=\{0\} \Leftrightarrow P$ possui largura constante.

\section{Demonstração.}

$(\Rightarrow)$ Por hipótese $P_{i}=P_{i+n}+\bar{w}_{P} U_{i}$

$$
\begin{aligned}
h_{i}+h_{i+n} & = \\
& =\left[P_{i}, V_{i}\right]+\left[P_{i+n}, V_{i+n}\right] \\
& =\left[P_{i}-P_{i+n}, V_{i}\right] \\
& =\left[\bar{w}_{P} U_{i}, V_{i}\right] \\
& =\bar{w}_{P}\left[U_{i}, V_{i}\right]=\bar{w}_{P}(\text { constante })
\end{aligned}
$$

$(\Leftarrow)$ Por hipótese $\bar{w}_{P}$ é a largura de $P$, isto é $h_{i}+h_{i+n}=\bar{w}_{P}$. Utilizando também a equação (7.4) teremos

$$
\begin{aligned}
P_{i}-P_{i+n} & =h_{i} U_{i}+\left(\Delta_{i-1} h\right) \frac{V_{i}}{\left[V_{i-1}, V_{i}\right]}-\left(h_{i+n} U_{i+n}+\left(\Delta_{i+n-1} h\right) \frac{V_{i+n}}{\left[V_{i+n-1}, V_{i+n}\right]}\right) \\
& =\left(h_{i}+h_{i+n}\right) U_{i}+\left(\Delta_{i-1} h+\Delta_{i+n-1} h\right) \frac{V_{i}}{\left[V_{i-1}, V_{i}\right]} \\
& =\bar{w}_{P} U_{i}
\end{aligned}
$$


Capítulo 7. Cáustica de Wigner e Conjunto de Medida de Largura

Proposição 7.18. $P \in C_{U} . C M L C(P)$ tem comprimento-v zero.

Demonstração.

$$
\begin{aligned}
& \text { Como } r_{C M L C}=\frac{r_{i}+r_{i+n}-\bar{w}_{P}}{2} \text { temos } \\
& \qquad \begin{aligned}
L_{V}\left(C M L C\left(P_{i}\right)\right) & =\sum_{i=1}^{2 n} r_{C M L C}\left[U_{i}, \Delta_{i} U\right] \\
& =\sum_{i=1}^{2 n} \frac{r_{i}+r_{i+n}-\bar{w}_{P}}{2}\left[U_{i}, \Delta_{i} U\right] \\
& =\frac{1}{2}\left(2 L_{V}(P)-2 \bar{w}_{P} A(U)\right) \\
& =L_{V}(P)-\frac{L_{V}(P)}{A(U)} A(U)=0
\end{aligned}
\end{aligned}
$$

Proposição 7.19. $P \in C_{U}, C M L C(C M L C(P))=C M L C(P)$

Demonstração. Como CMLC é simétrico e possui comprimento-v zero temos

$$
\begin{aligned}
C M L C\left(C M L C\left(P_{i}\right)\right) & =\frac{C M L C\left(P_{i}\right)-C M L C\left(P_{i+n}\right)-\bar{w}_{P} U_{i}}{2} \\
& =\frac{C M L C\left(P_{i}\right)+C M L C\left(P_{i}\right)-0 \cdot U_{i}}{2} \\
& =\frac{2 C M L C\left(P_{i}\right)}{2} \\
& =C M L C\left(P_{i}\right)
\end{aligned}
$$

Proposição 7.20. $P \in C_{U}, C M L C(P(c))=C M L C(P)$.

\section{Demonstração.}

Como $L_{V}(P(c))=\sum_{i}^{2 n}\left(r_{i}+c\right)\left[U_{i}, U_{i+1}\right]=L_{V}(P)+2 c A(U)$, teremos portanto $\bar{w}_{P(c)}=\bar{w}_{P}+2 c$. Assim 
Capítulo 7. Cáustica de Wigner e Conjunto de Medida de Largura

$$
\begin{aligned}
C M L C\left(P_{i}(c)\right) & =\frac{P_{i}(c)-P_{i+n}(c)-\bar{w}_{P(c)} U_{i}}{2} \\
& =\frac{P_{i}+c U_{i}-P_{i+n}-c U_{i+n}-\bar{w}_{P(c)} U_{i}}{2} \\
& =\frac{P_{i}+c U_{i}-P_{i+n}+c U_{i}-\bar{w}_{P(c)} U_{i}}{2} \\
& =\frac{P_{i}-P_{i+n}+2 c U_{i}-\left(\bar{w}_{P}+2 c\right) U_{i}}{2} \\
& =\frac{P_{i}-P_{i+n}-\bar{w}_{P} U_{i}}{2} \\
& =C M L C\left(P_{i}\right)
\end{aligned}
$$

\section{3}

Decomposição do polígono $\mathbf{P}$ como soma de Caústica de Wigner, CMLC e bola unitária.

Proposição 7.21. $P \in C_{U}, P_{i}=C W\left(P_{i}\right)+C M L C\left(P_{i}\right)+\frac{\bar{w}_{P}}{2} U_{i}, \forall i \in$ $\{1, \ldots, 2 n\}$ e $h_{P}=h_{C W(P)}+h_{C M L C(P)}+\frac{\bar{w}_{P}}{2}$

Corolário 7.22. $P \in C_{U}, P_{i}=C W\left(P_{i}\right)+C M L C\left(P_{i}\right), \forall i \in\{1, \ldots, 2 n\} e$ $h_{P}=h_{C W(P)}+h_{C M L C(P)} \Leftrightarrow L_{V}(P)=0$

É fácil ver que os resultados acima são consequência do fato que

$$
P_{i}=\frac{P_{i}+P_{i+n}}{2}+\frac{P_{i}-P_{i+n}}{2} .
$$

\section{4}

\section{Cúspides CMLC e Cáustica de Wigner}

Definição 7.23. Seja $P$ um polígono em $C_{U}$. Dizemos que um vértice $P_{i}$ em $P$ é um cúspide se os seus lado vizinhos(não degenerados) possuem orientação oposta, isto é, os segmentos anterior e posterior a $P_{i}$ possuem raios de curvatura com sinais contrários.

Para mais detalhes dessa definição ver [7].

Teorema 7.24. Considere o polígono $P$ em $C_{U}$. Então o número de cúspides de $C M L C(P)$ é múltiplo de quatro.

Demonstração. O número de cúspides de $\mathrm{CMLC}(\mathrm{P})$ é igual ao número de trocas de sinal do raio de curvatura em segmentos consecutivos. Como $r_{C M L C\left(P_{i}\right)}=\left(r_{i}+r_{i+n}-\bar{w}_{P}\right) / 2$ teremos $r_{C M L C\left(P_{i}\right)}=r_{C M L C\left(P_{i+n}\right)}$. Uma vez que $L_{V}(C M L C(P))=0$ temos 


$$
r_{C M L C\left(P_{1}\right)}\left[U_{1}, U_{2}\right]+r_{C M L C\left(P_{2}\right)}\left[U_{2}, U_{3}\right]+\ldots+r_{C M L C\left(P_{n}\right)}\left[U_{n}, U_{n+1}\right]=0
$$

e

$r_{C M L C\left(P_{n+1}\right)}\left[U_{n+1}, U_{n+2}\right]+r_{C M L C\left(P_{n+2}\right)}\left[U_{n+2}, U_{n+3}\right]+\ldots+r_{C M L C\left(P_{2 n}\right)}\left[U_{2 n}, U_{2 n+1}\right]=0$.

Das igualdades anteriores e pelo fato de $\left[U_{i}, U_{i+1}\right]>0, \forall i \in\{1, \ldots, 2 n\}$, concluímos que existe pelo menos um $k \in\{1, \ldots, n\}$ tal que $r_{C M L C\left(P_{k}\right)}<0$ assim o número de trocas de sinal de $k$ até $k+n$ deve ser par e pelo menos 2 . O mesmo vale de $k+n$ até $k+2 n$. Temos o desejado.

Teorema 7.25. Considere o polígono $P$ em $C_{U}$. Então o número de cúspides de $C W(P)$ é $2 k, k$ impar.

Demonstração. Observe que $r_{C W\left(P_{i}\right)}=\frac{r_{i}-r_{i+n}}{2}$. Como $r_{C W\left(P_{i}\right)}=$ $-r_{C W\left(P_{i+n}\right)}$ então o número de trocas de sinal do raio de curvatura em segmentos consecutivos de 1 até $n+1$ é ímpar, $k$ por exemplo, e igual ao número de trocas de sinal de $n+1$ até $2 n+1$. Temos o desejado.

\section{5}

\section{Áreas com sinal de CW e CMLC}

Definição 7.26. Sejam $P$ e $Q$ polígonos definidos em $C_{U}$. Definimos como área mista dos polígonos $P$ e $Q$,

$$
A(P, Q)=\frac{1}{2} \sum_{i=1}^{2 n}\left[P_{i}, \Delta_{i} Q\right] .
$$

Teorema 7.27. (Desigualdade de Minkowski) Sejam $P$ e $Q$ polígonos simples e convexos em $C_{U}$ então

$$
A(P, Q)^{2} \geq A(P) A(Q)
$$

onde $A(P), A(Q)$ e $A(P, Q)$ são respectivamente as áreas das curvas e área mista.

Note que se fizermos $Q=U$, U bola unitária, temos pela equação (8.5) 
Capítulo 7. Cáustica de Wigner e Conjunto de Medida de Largura

$$
A(P, U)=\frac{1}{2} \sum_{i=1}^{2 n}\left[P_{i}, \Delta_{i} U\right]=\frac{1}{2} \sum_{i=1}^{2 n}\left[U_{i}, \Delta_{i} P\right]=\frac{1}{2} \sum_{i=1}^{2 n} r_{i}\left[U_{i}, \Delta_{i} U\right]=\frac{L_{V}(P)}{2}
$$

isto é, temos a desigualdade isoperimétrica de Minkowski,

$$
L_{V}^{2} \geq 4 A(U) A(P)
$$

Proposição 7.28. Seja o polígono $P \in C_{U}$ então $\tilde{\mathrm{A}}(C W(P))<0$, $\tilde{\mathrm{A}}(C M L C(P))<0$.

\section{Demonstração.}

Ver capítulo 9 seção (9.1).

\section{6}

\section{Evolutas}

Definição 7.29. Definimos $U$-evoluta do polígono $P$ em $C_{P}$ como sendo o polígono $E(P)$ tal que

$$
E\left(P_{i}\right)=P_{i}-r_{i} U_{i}=P_{i+1}-r_{i} U_{i+1}, i=1, \ldots, 2 n .
$$

onde $r=\left(r_{i}\right)_{i \in \mathbb{N}}$ é o raio de curvatura de $P$.

Proposição 7.30. $P \in C_{U} \cdot P(c)$ e $P$ possuem a mesma $U$-evoluta.

Demonstração. Como $\Delta_{i}(P(c))=\left(r_{i}+c\right) \Delta_{i} U$ temos que a evoluta do paralelo $P(c)$ é $E\left(P_{i}(c)\right)=P_{i}(c)-\left(r_{i}+c\right) U_{i}=P_{i}+c U_{i}-r_{i} U_{i}-c U_{i}=E\left(P_{i}\right)$

Proposição 7.31. A $U$-evoluta de $P \in C_{U}$ possui comprimento- $U$ com sinal igual a zero.

Demonstração. Basta observar que $\Delta_{i} E(P)=\left(-\Delta_{i} r\right) U_{i+1}$. Assim temos

$$
L_{U}(E(P))=\sum_{i=1}^{2 n}-\Delta_{i} r=0
$$

Proposição 7.32. Seja $P \in C_{U} . A V$-função suporte da $U$-evoluta de $P$ é $h_{E_{P_{i}}}=-\frac{\Delta_{i-1} h}{\left[V_{i-1}, V_{i}\right]}$, onde $h$ é a $U-$ função suporte de $P$. 
Demonstração. Considere $r$ o raio de curvatura de $P$. Como $E\left(P_{i}\right)=$ $P_{i}-r_{i} U_{i}$ então $\Delta_{i} E(P)=\left(-\Delta_{i} r\right) U_{i+1}=\frac{\Delta_{i} r}{\left[V_{i}, V_{i+1}\right]} \Delta_{i} V$.

Portanto

$$
\begin{aligned}
h_{E_{P_{i}}} & =\left[E\left(P_{i}\right), U_{i}\right] \\
& =\left[P_{i}-r_{i} U_{i}, U_{i}\right] \\
& =\left[P_{i}, U_{i}\right] \\
& =\left[h_{i} U_{i}+\left(\Delta_{i-1} h\right) \frac{V_{i}}{\left[V_{i-1}, V_{i}\right]}, U_{i}\right] \\
& =-\frac{\Delta_{i-1} h}{\left[V_{i-1}, V_{i}\right]}
\end{aligned}
$$

Proposição 7.33. Seja $P \in C_{U}$. A U-função suporte da V-evoluta da $U$-evoluta, isto é, a dupla evoluta de $P$ é

$$
h_{E\left(E\left(P_{i+1}\right)\right)}=-\frac{1}{\left[u_{i}, u_{i+1}\right]} \nabla_{i}\left(\frac{\Delta_{i} h}{\left[v_{i}, v_{i+1}\right]}\right),
$$

onde h é a $U$-função suporte de $P$.

Demonstração. Definimos

$$
E\left(E\left(P_{i+1}\right)\right)=E\left(P_{i+1}\right)-\frac{\Delta_{i} r}{\left[V_{i}, V_{i+1}\right]} V_{i+1}=E\left(P_{i}\right)-\frac{\Delta_{i} r}{\left[V_{i}, V_{i+1}\right]} V_{i},
$$

então

$$
\begin{aligned}
\Delta_{i} E_{E(P)} & =-\nabla_{i}\left(\frac{\Delta_{i} r}{\left[V_{i}, V_{i+1}\right]}\right) V_{i} \\
& =-\nabla_{i}\left(\frac{\Delta_{i} r}{\left[V_{i}, V_{i+1}\right]}\right) \frac{1}{\left[U_{i}, U_{i+1}\right]} \Delta_{i} U .
\end{aligned}
$$

Portanto 
Capítulo 7. Cáustica de Wigner e Conjunto de Medida de Largura Constante-Discreto

$$
\begin{aligned}
h_{E\left(E\left(P_{i+1}\right)\right)} & =\left[E\left(E\left(P_{i+1}\right)\right), V_{i}\right] \\
& =\left[E\left(P_{i}\right)-\frac{\Delta_{i} r}{\left[V_{i}, V_{i+1}\right]} V_{i}, V_{i}\right] \\
& =\left[E\left(P_{i}\right), V_{i}\right] \\
& =\left[P_{i}-r_{i} U_{i}, V_{i}\right] \\
& =h_{i}-\left(h_{i}-\frac{1}{\left[U_{i}, U_{i+1}\right]} \nabla_{i}\left(\frac{\Delta_{i} h}{\left[V_{i}, V_{i+1}\right]}\right)\right) \\
& =-\frac{1}{\left[U_{i}, U_{i+1}\right]} \nabla_{i}\left(\frac{\Delta_{i} h}{\left[V_{i}, V_{i+1}\right]}\right)
\end{aligned}
$$

Proposição 7.34. Seja $P \in C_{U}$. Então $E\left(C W\left(P_{i}\right)\right)=C W\left(E\left(P_{i}\right)\right) e$ $E\left(C M L C\left(P_{i}\right)\right)=C M L C\left(E\left(P_{i}\right)\right)$. Isto é, evolutas comutam com CW e CMLC.

\section{Demonstração.}

$$
\begin{aligned}
E\left(C W\left(P_{i}\right)\right)= & C W\left(P_{i}\right)-r_{C W_{i}} U_{i} \\
= & \frac{P_{i}+P_{i+n}}{2}-\frac{r_{i}-r_{i+n}}{2} U_{i} \\
= & \frac{P_{i}-r_{i} U_{i}+P_{i+n}-r_{i+n} U_{i+n}}{2} \\
= & \frac{E\left(P_{i}\right)+E\left(P_{i+n}\right)}{2} \\
& =C W\left(E\left(P_{i}\right)\right)
\end{aligned}
$$

$$
\begin{aligned}
E\left(C M L C\left(P_{i}\right)\right)= & C M L C\left(P_{i}\right)-r_{C M L C_{i}} U_{i} \\
= & \frac{P_{i}-P_{i+n}-\bar{w}_{P} U_{i}}{2}-\frac{r_{i}+r_{i+n}-\bar{w}_{P}}{2} U_{i} \\
= & \frac{P_{i}-r_{i} U_{i}-\left(P_{i+n}-r_{i+n} U_{i+n}\right)-\bar{w}_{E_{P}} U_{i}}{2} \\
= & \frac{E\left(P_{i}\right)-E\left(P_{i+n}\right)-\bar{w}_{E_{P}} U_{i}}{2} \\
& =C M L C\left(E\left(P_{i}\right)\right)
\end{aligned}
$$

Observe que nesse caso $\bar{w}_{E_{P}}=\frac{L_{U}\left(E_{P}\right)}{A(U)}=0$ 


\section{7}

\section{Exemplos}

Exemplo 7.35. Seja a bola unitária $U$, o hexágono descrito no exemplo (2.3). Considere o polígono $P$ em $C_{U}$ com raio de curvatura $r=\left(r_{1}, r_{2}, r_{3}, r_{4}, r_{5}, r_{6}\right)$. Como $P$ é fechado então $\sum_{i=1}^{6} \Delta_{i} P=\sum_{i=1}^{6} r_{i} \Delta_{i} U=0$, o que implica $r_{1}\left(U_{2}-U_{1}\right)+r_{2}\left(U_{3}-U_{2}\right)+r_{3}\left(U_{4}-U_{3}\right)+r_{4}\left(U_{5}-U_{4}\right)+r_{5}\left(U_{6}-U_{5}\right)+r_{6}\left(U_{1}-U_{6}\right)=0$.

Substituindo as coordenadas da bola unitária $U$ teremos:

$$
r_{1}+r_{2}-r_{4}-r_{5}=0 \text { e } r_{1}-r_{2}-2 r_{3}-r_{4}+r_{5}+2 r_{6}=0
$$

Esse sistema possui infinitas soluções e duas variáveis livres. Portanto a dimensão de $C_{U}$ é 4 . No caso geral a dimensão de $C_{U}$ é $2 n-2$, ver [7].

Exemplo 7.36. Seja a bola unitária $U$, o hexágono descrito no exemplo (2.3). Considere o polígono $P \in C_{U}$ de raio de curvatura $r=\left(\frac{1}{2}, 1, \frac{1}{2}, \frac{3}{4}, \frac{3}{4}, \frac{3}{4}\right)$, conforme exemplo (7.34). Vamos calcular $C W(P)$ e $C M L C(P)$. Ver figura 7.1.

$$
\begin{gathered}
L_{V}(P)=\sum_{i=1}^{6} r_{i}\left[U_{i}, U_{i+1}\right]=\frac{\sqrt{3}}{2} \sum_{i=1}^{6} r_{i}=\frac{\sqrt{3}}{2}\left(\frac{1}{2}+1+\frac{1}{2}+\frac{3}{4}+\frac{3}{4}+\frac{3}{4}\right)=\frac{17 \sqrt{3}}{8} \\
e \\
A(U)=\frac{1}{2} \sum_{i=1}^{6}\left[U_{i}, U_{i+1}\right]=3 \frac{\sqrt{3}}{2} .
\end{gathered}
$$

$\operatorname{Assim} \bar{w}_{P}=\frac{L_{V}(P)}{A(U)}=\frac{17}{12}$ e $C M L C\left(P_{i}\right)=C_{i}=\left(P_{i}-P_{i+n}-\frac{17}{12} U_{i}\right) / 2$. Os valores de $U_{i}$ podem ser encontrados no exemplo (7.4).

Como $P_{i+1}=r_{i} \Delta_{i} U+P_{i}$ e $P_{1}=(0,0)$ então teremos $P_{2}=\left(\frac{-\sqrt{3}}{4}, \frac{1}{4}\right)$, $P_{3}=\left(\frac{-3 \sqrt{3}}{4},-\frac{1}{4}\right), P_{4}=\left(\frac{-3 \sqrt{3}}{4},-\frac{3}{4}\right), P_{5}=\left(\frac{-3 \sqrt{3}}{8},-\frac{9}{8}\right), P_{6}=\left(0,-\frac{3}{4}\right)$.

Portanto $C_{1}=\left(\frac{\sqrt{3}}{48}, \frac{1}{48}\right), C_{2}=\left(\frac{\sqrt{3}}{16},-\frac{1}{48}\right), C_{3}=\left(-\frac{\sqrt{3}}{48},-\frac{5}{48}\right), C_{4}=\left(-\frac{\sqrt{3}}{48},-\frac{1}{48}\right)$, $C_{5}=\left(-\frac{\sqrt{3}}{16}, \frac{1}{48}\right), C_{6}=\left(\frac{\sqrt{3}}{48}, \frac{5}{48}\right)$. Observe que $C_{1}=-C_{4}, C_{2}=-C_{5}, C_{3}=-C_{6}$, isto é, CMLC é simétrico. Note também que $r_{C M L C\left(P_{1}\right)}=-1 / 12=r_{C M L C\left(P_{4}\right)}$ $r_{C M L C\left(P_{2}\right)}=1 / 6=r_{C M L C\left(P_{5}\right)}, r_{C M L C\left(P_{3}\right)}=-1 / 12=r_{C M L C\left(P_{6}\right)}$. Assim podemos ver

$L_{V}(C M L C(P))=\sum_{i=1}^{6} r_{C M L C\left(P_{i}\right)}\left[U_{i}, U_{i+1}\right]=\frac{\sqrt{3}}{2}\left(-\frac{1}{12}+\frac{1}{6}-\frac{1}{12}-\frac{1}{12}+\frac{1}{6}-\frac{1}{12}\right)=0$ 
Capítulo 7. Cáustica de Wigner e Conjunto de Medida de Largura

Como $C W\left(P_{i}\right)=\frac{P_{i}+P_{i+n}}{2}$ teremos $C W\left(P_{1}\right)=C W\left(P_{4}\right)=\left(\frac{-3 \sqrt{3}}{8}, \frac{-3}{8}\right)$, $C W\left(P_{2}\right)=C W\left(P_{5}\right)=\left(\frac{-5 \sqrt{3}}{16}, \frac{-7}{16}\right), C W\left(P_{3}\right)=C W\left(P_{6}\right)=\left(\frac{-3 \sqrt{3}}{8}, \frac{-1}{2}\right)$. Podemos ver que também que $r_{C W(P)}=\left(-\frac{1}{8}, \frac{1}{8},-\frac{1}{8}, \frac{1}{8},-\frac{1}{8}, \frac{1}{8}\right)$ e $L_{V}(C W(P))=$ 0 .

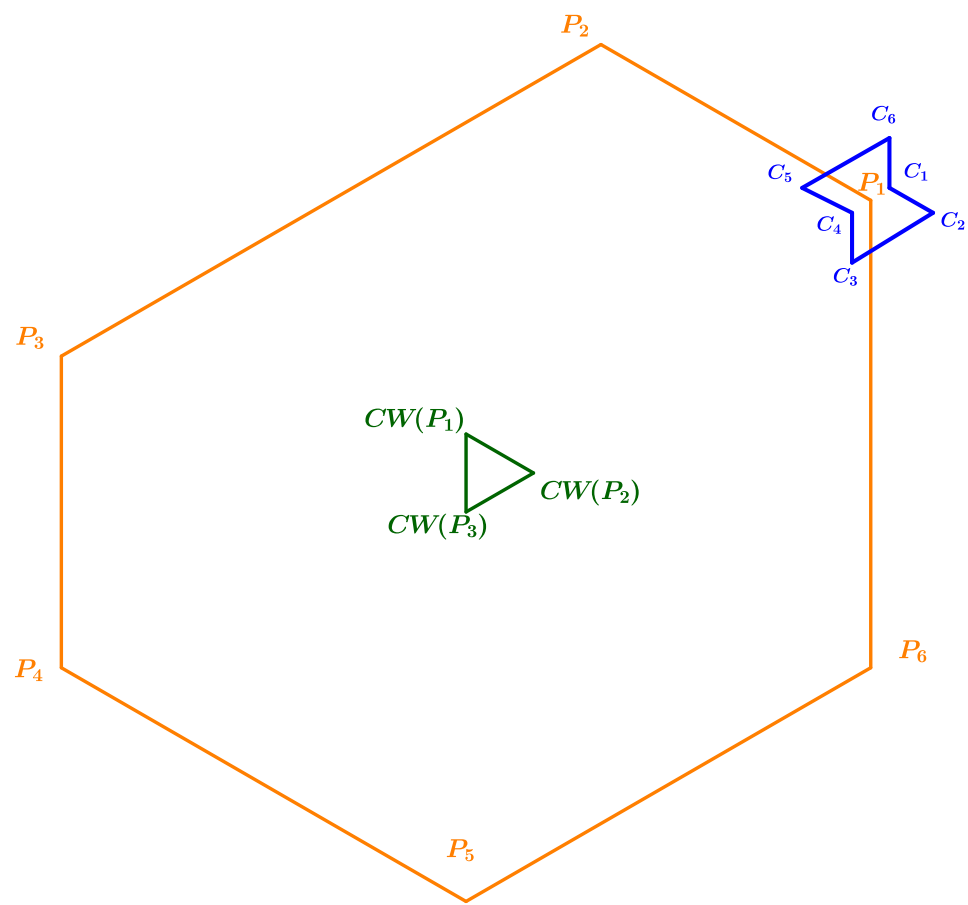

Figura 7.1: Polígono P, Cáustica de Wigner e CMLC C 


\section{8 \\ Igualdade Isoperimétrica em Plano Normado - Discreto}

Teorema 8.1. Seja $P=\left\{P_{1}, \ldots, P_{2 n}\right\}$ polígono em $C_{U}$ no plano $\left(\mathbb{R}^{2}, U\right)$, onde $U=\left\{U_{1}, \ldots, U_{2 n}\right\}$ é a bola unitária e $P_{i+1}-P_{i}$ é paralelo a $U_{i+1}-U_{i}$. Então

$$
L_{V}^{2}(P)=4 A_{U} \tilde{\mathrm{A}}_{P}-8 A_{U} \tilde{\mathrm{A}}_{C W(P)}-4 A_{U} \tilde{\mathrm{A}}_{C M L C(P)},
$$

onde $L_{V}(P)$ indica o $V$-comprimento com sinal do polígono $P, A_{U}$ indica área da bola unitária e $\widetilde{\mathrm{A}}(P), \widetilde{\mathrm{A}}_{C W(P)}$ e $\widetilde{\mathrm{A}}_{C M L C(P)}$ indicam respectivamente as áreas com sinal de $P, C W(P)$ e $C M L C(P)$.

\section{Demonstração.}

Pela proposição (7.20) podemos escrever $P_{i}=\frac{P_{i}+P_{i+n}}{2}+$ $\frac{P_{i}-P_{i+n}-\bar{w}_{P} U_{i}}{2}+\frac{\bar{w}_{P}}{2} U_{i}$, isto é, $P_{i}=C W\left(P_{i}\right)+C M L C\left(P_{i}\right)+\frac{\bar{w}_{P}}{2} U_{i}$. Utilizando o operador diferença temos $\Delta_{i} P=\Delta_{i} C W(P)+\Delta_{i} C M L C(P)+$ $\frac{\bar{w}_{P}}{2} \Delta_{i} U$.

A seguir, temos três igualdades importantes para a prova.

$$
\begin{aligned}
\sum_{i=1}^{2 n}\left[C W\left(P_{i}\right), \Delta_{i} C M L C(P)\right]= & -\sum_{i=1}^{2 n}\left[\Delta_{i} C W(P), C M L C\left(P_{i}\right)\right] \\
\sum_{i=1}^{2 n} \frac{\bar{w}_{P}}{2}\left[C W\left(P_{i}\right), \Delta_{i} U\right] & =\sum_{i=1}^{2 n} \frac{\bar{w}_{P}}{2}\left[U_{i}, \Delta_{i} C W(P)\right] \\
\sum_{i=1}^{2 n} \frac{\bar{w}_{P}}{2}\left[C M L C\left(P_{i}\right), \Delta_{i} U\right] & =\sum_{i=1}^{2 n} \frac{\bar{w}_{P}}{2}\left[U_{i}, \Delta_{i} C M L C(P)\right]
\end{aligned}
$$

Vamos calcular a área com sinal do polígono $\mathrm{P}$ utilizando a proposição (7.20) e as igualdades (8.2), (8.3) e (8.4). Iremos utilizar também a proposição (7.27) que diz $\widetilde{\mathrm{A}}(C W(P))<0$ e $\widetilde{\mathrm{A}}(C M L C(P))<0$. 


$$
\begin{aligned}
& \widetilde{\mathrm{A}}(P)= \\
& =\frac{1}{2} \sum_{i=1}^{2 n}\left[P_{i}, \Delta_{i} P\right] \\
& =\frac{1}{2} \sum_{i=1}^{2 n}\left[C W\left(P_{i}\right)+C M L C\left(P_{i}\right)+\frac{\bar{w}_{P}}{2} U_{i}, \Delta_{i} C W(P)+\Delta_{i} C M L C(P)+\frac{\bar{w}_{P}}{2} \Delta_{i} U\right] \\
& =\frac{1}{2} \sum_{i=1}^{2 n}\left[C W\left(P_{i}\right), \Delta_{i} C W(P)\right]+\frac{1}{2} \sum_{i=1}^{2 n}\left[C W\left(P_{i}\right), \Delta_{i} C M L C(P)\right] \\
& +\frac{1}{2} \sum_{i=1}^{2 n} \frac{\bar{w}_{P}}{2}\left[C W\left(P_{i}\right), \Delta_{i} U\right]+\frac{1}{2} \sum_{i=1}^{2 n}\left[C M L C\left(P_{i}\right), \Delta_{i} C W(P)\right] \\
& +\frac{1}{2} \sum_{i=1}^{2 n}\left[C M L C\left(P_{i}\right), \Delta_{i} C M L C(P)\right]+\frac{1}{2} \sum_{i=1}^{2 n} \frac{\bar{w}_{P}}{2}\left[C M L C\left(P_{i}\right), \Delta_{i} U\right] \\
& +\frac{1}{2} \sum_{i=1}^{2 n} \frac{\bar{w}_{P}}{2}\left[U_{i}, \Delta_{i} C W(P)\right]+\frac{1}{2} \sum_{i=1}^{2 n} \frac{\bar{w}_{P}}{2}\left[U_{i}, \Delta_{i} C M L C(P)\right] \\
& +\frac{1}{2} \sum_{i=1}^{2 n} \frac{\bar{w}_{P}^{2}}{4}\left[U_{i}, \Delta_{i} U\right] \\
& \Rightarrow \\
& \widetilde{\mathrm{A}}(P)=2 \widetilde{\mathrm{A}}_{C W(P)}+\widetilde{\mathrm{A}}_{C M L C(P)}+\frac{\bar{w}_{P}^{2}}{4} A(U)+\sum_{i=1}^{2 n} \frac{\bar{w}_{P}}{2}\left[C W\left(P_{i}\right), \Delta_{i} U\right] \\
& +\sum_{i=1}^{2 n} \frac{\bar{w}_{P}}{2}\left[C M L C\left(P_{i}\right), \Delta_{i} U\right] \\
& =2 \widetilde{\mathrm{A}}_{C W(P)}+\widetilde{\mathrm{A}}_{C M L C(P)}+\frac{\bar{w}_{P}^{2}}{4} A(U)+\sum_{i=1}^{2 n} \frac{\bar{w}_{P}}{2}\left[C W\left(P_{i}\right)+C M L C\left(P_{i}\right), \Delta_{i} U\right] \\
& =2 \widetilde{\mathrm{A}}_{C W(P)}+\tilde{\mathrm{A}}_{C M L C(P)}+\frac{\bar{w}_{P}^{2}}{4} A(U)+\sum_{i=1}^{2 n} \frac{\bar{w}_{P}}{2}\left[P_{i}-\frac{\bar{w}_{P}}{2} U_{i}, \Delta_{i} U\right] \\
& =2 \widetilde{\mathrm{A}}_{C W(P)}+\widetilde{\mathrm{A}}_{C M L C(P)}+\frac{\bar{w}_{P}^{2}}{4} A(U) \\
& +\frac{\bar{w}_{P}}{2}\left(\sum_{i=1}^{2 n}\left[P_{i}, \Delta_{i} U\right]-\frac{\bar{w}_{P}}{2} \sum_{i=1}^{2 n}\left[U_{i}, \Delta_{i} U\right]\right) \\
& =2 \widetilde{\mathrm{A}}_{C W(P)}+\widetilde{\mathrm{A}}_{C M L C(P)}+\frac{\bar{w}_{P}^{2}}{4} A(U) \\
& +\frac{\bar{w}_{P}}{2}\left(L_{V}(P)-\frac{\bar{w}_{P}}{2} 2 A(U)\right) \\
& =2 \widetilde{\mathrm{A}}_{C W(P)}+\tilde{\mathrm{A}}_{C M L C(P)}+\frac{\left(L_{V}(P)\right)^{2}}{4 A(U)} \\
& +\frac{\bar{w}_{P}}{2}\left(L_{V}(P)-L_{V}(P)\right) \\
& \Rightarrow \\
& \tilde{\mathrm{A}}(P)=2 \tilde{\mathrm{A}}_{C W(P)}+\tilde{\mathrm{A}}_{C M L C(P)}+\frac{L_{V}^{2}(P)}{4 A(U)}
\end{aligned}
$$

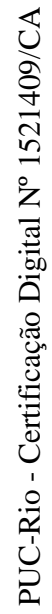




$$
\begin{aligned}
\widetilde{\mathrm{A}}_{P} & =2 \widetilde{\mathrm{A}}_{C W(P)}+\widetilde{\mathrm{A}}_{C M L C(P)}+\frac{L_{V}^{2}(P)}{4 A_{U}} \\
L_{V}^{2}(P) & =4 A_{U} \widetilde{\mathrm{A}}_{P}-8 A_{U} \tilde{\mathrm{A}}_{C W(P)}-4 A_{U} \tilde{\mathrm{A}}_{C M L C(P)}
\end{aligned}
$$

Corolário 8.2. Seja $P=\left\{P_{1}, \ldots, P_{2 n}\right\}$ polígono simples, fechado, convexo no plano $\left(\mathbb{R}^{2}, U\right)$, onde $U=\left\{U_{1}, \ldots, U_{2 n}\right\}$ é a bola unitária e $P_{i+1}-P_{i}$ é paralelo a $U_{i+1}-U_{i}$. Então

$$
L_{V}^{2}(P)=4 A_{U} A_{P}+8 A_{U}\left|\tilde{\mathrm{A}}_{C W(P)}\right|+4 A_{U}\left|\tilde{\mathrm{A}}_{C M L C(P)}\right|
$$

onde $L_{V}(P)$ indica o comprimento do polígono $P$ na norma dual, $A_{U} e$ $A_{P}$ indicam respectivamente as áreas da bola unitária e do polígono $P e$ $\tilde{\mathrm{A}}_{C W(P)}$ e $\tilde{\mathrm{A}}_{C M L C(P)}$ indicam respectivamente as áreas com sinal de $C W(P)$ e $C M L C(P)$.

Corolário 8.3. Seja $P=\left\{P_{1}, \ldots, P_{2 n}\right\}$ polígono simples, fechado, convexo no plano $\left(\mathbb{R}^{2}, U\right)$, onde $U=\left\{U_{1}, \ldots, U_{2 n}\right\}$ é a bola unitária e $P_{i+1}-P_{i}$ é paralelo a $U_{i+1}-U_{i}$. Então

$$
L_{V}^{2}(P) \geq 4 A_{U} A_{P}+8 A_{U}\left|\tilde{\mathrm{A}}_{C W(P)}\right|
$$

onde $L_{V}(P)$ indica o comprimento do polígono $P$ na norma dual, $A_{U}$ e $A_{P}$ indicam respectivamente as áres da bola unitária e do polígono $P$ e $\widetilde{\mathrm{A}}_{C W(P)}$ indica a área orientada de $C W(P)$. A igualdade ocorre se, e só se, o polígono $P$ tem largura constante na norma $U$.

Corolário 8.4 (Desigualdade Isoperimétrica Clássica em plano normado.). Seja $P=\left\{P_{1}, \ldots, P_{2 n}\right\}$ polígino simples, fechado, convexo no plano $\left(\mathbb{R}^{2}, U\right)$, onde $U=\left\{U_{1}, \ldots, U_{2 n}\right\}$ é a bola unitária e $P_{i+1}-P_{i}$ é paralelo a $U_{i+1}-U_{i}$. Então

$$
L_{V}^{2}(P) \geq 4 A_{U} A_{P}
$$

onde $L_{V}(P)$ indica o comprimento do polígono $P$ na norma dual, $A_{U}$ e $A_{P}$ indicam respectivamente as áreas da bola unitária e do polígono P. A igualdade ocorre se, e só se,o polígono $P$ é simétrico e tem largura constante, isto é, múltiplo da bola. 
Corolário 8.5. Seja $P=\left\{P_{1}, \ldots, P_{2 n}\right\}$ polígono simples, fechado, convexo no plano $\left(\mathbb{R}^{2}, U\right)$, onde $U=\left\{U_{1}, \ldots, U_{2 n}\right\}$ é a bola unitária e $P_{i+1}-P_{i}$ é paralelo a $U_{i+1}-U_{i}$. Então

$$
L_{V}^{2}(P) \geq 4 A_{U} A_{P}+4 A_{U}\left|\tilde{\mathrm{A}}_{C M L C(P)}\right|,
$$

onde $L_{V}(P)$ indica o comprimento do polígono $P$ na norma dual, $A_{U}$ e $A_{P}$ indicam respectivamente as áreas da bola unitária e do polígono $P$ e $\widetilde{\mathrm{A}}_{C M L C(P)}$ indica a área orientada de $C M L C(P)$.

\section{1}

\section{Exemplo}

Exemplo 8.6. Podemos verificar o Corolário (8.2) caso discreto, utilizando o exemplo (7.35). Ver figura 7.1. Onde $L_{V}(P)=\frac{17 \sqrt{3}}{8}, A(U)=\frac{3 \sqrt{3}}{2}$, $A(P)=\frac{47 \sqrt{3}}{64}, \tilde{\mathrm{A}}_{C W(P)}=-\frac{\sqrt{3}}{256}, \tilde{\mathrm{A}}_{C M L C(P)}=-\frac{\sqrt{3}}{96}$.

Substituindo:

$$
\begin{aligned}
A(U)\left(4 A_{P}+8\left|\tilde{\mathrm{A}}_{C W(P)}\right|+4\left|\tilde{\mathrm{A}}_{C M L C(P)}\right|\right) & =\frac{3 \sqrt{3}}{2}\left(4 \cdot \frac{47 \sqrt{3}}{64}+8 \cdot \frac{\sqrt{3}}{256}+4 \cdot \frac{\sqrt{3}}{96}\right) \\
& =\frac{867}{64}=\frac{17^{2}(\sqrt{3})^{2}}{8^{2}}=L_{V}^{2}(P)
\end{aligned}
$$




\section{9 \\ Ciclóides e Base Ortonormal - Discreto}

Ciclóides clássicas tem a propriedade de que sua dupla evoluta é homotética a ela mesma. Considerando esta mesma propriedade em um plano normado $\left(\mathbb{R}^{2}, U\right)$, onde $U$ é bola unitária poligonal, temos a seguinte definição (proposição (7.32)):

Definição 9.1. Definimos como Ciclóide discreta $F \in C_{U}$ o polígono cuja função suporte $h_{F_{i}}$ satisfaz a equação

$$
-\frac{1}{\left[U_{i}, U_{i+1}\right]} \nabla_{i}\left(\frac{\Delta_{i} h_{F}}{\left[V_{i}, V_{i+1}\right]}\right)=\lambda h_{F_{i}}, \lambda \in \mathbb{R}
$$

que é a discretização natural de segunda ordem da Equação Diferencial de Sturm-Liouville exibida em [6].

Definição 9.2. Dizemos que $\lambda$ é autovalor da equação (9.1) se existe $U$-função suporte $h$ que satisfaz tal equação. A função $h$ é chamada de autovetor.

Seja $P \in C_{U}$. Pela equação (6.4) existe uma correspondência entre $P$ e $h$ de forma que para cada $P$ existe uma e apenas uma função suporte, assim vamos a partir de agora apresentar $C_{U}$ como espaço das funções suporte e vamos muni-lo com o produto interno.

Definição 9.3. Sejam $h, \bar{h} \in C_{U}$ então definimos o produto interno

$$
\langle h, \bar{h}\rangle_{U}=\sum_{k=1}^{2 n} h_{k} \bar{h}_{k}\left[U_{k}, U_{k+1}\right]
$$

onde $h$ e $\bar{h}$ correspondem a funções suportes de dois polígonos em $C_{U}$.

Lema 9.4. Seja $h \in C_{U}$. A transformação linear $T$ tal que

$$
T h=\left(T h_{i}\right)_{i \in \mathbb{N}}=\left(-\frac{1}{\left[U_{i}, U_{i+1}\right]} \nabla_{i}\left(\frac{\Delta_{i} h}{\left[V_{i}, V_{i+1}\right]}\right)\right)_{i \in \mathbb{N}},
$$

definida em $C_{U}$ é auto-adjunta com respeito ao produto interno (9.2). 


\section{Demonstração.}

$$
\begin{aligned}
\langle T h, s\rangle_{U} & =\sum_{i=1}^{2 n} s_{i} T h_{i}\left[U_{i}, U_{i+1}\right] \\
& =\sum_{i=1}^{2 n}-s_{i}\left(\frac{1}{\left[U_{i}, U_{i+1}\right]} \nabla_{i}\left(\frac{\Delta_{i} h}{\left[V_{i}, V_{i+1}\right]}\right)\right)\left[U_{i}, U_{i+1}\right] \\
& =\sum_{i=1}^{2 n}-s_{i} \nabla_{i}\left(\frac{\Delta_{i} h}{\left[V_{i}, V_{i+1}\right]}\right) \\
& =\sum_{i=1}^{2 n} \frac{\Delta_{i} s \Delta_{i} h}{\left[V_{i}, V_{i+1}\right]} \\
& =\sum_{i=1}^{2 n}-h_{i} \nabla_{i}\left(\frac{\Delta_{i} s}{\left[V_{i}, V_{i+1}\right]}\right) \\
& =\sum_{i=1}^{2 n} h_{i} T s_{i}\left[U_{i}, U_{i+1}\right]=\langle T s, h\rangle_{U}
\end{aligned}
$$

Suponhamos que o raio de curvatura $r$ de $P$ satisfaça a equação (10.1) com $\lambda \neq 1$, então $h_{i}=\frac{r_{i}}{1-\lambda}$ satisfaz (10.1). Reciprocamente se a função suporte $h$ do polígono $P$ satisfaz (10.1) então pela equação (7.6), $r_{i}=h_{i}(1-\lambda)$ também satisfaz (10.1).

Segundo [7], $\lambda=1$ indica as ciclóides discretas abertas que, portanto, não podem ser representadas por funções suporte periódicas. Assim para $\lambda \neq 1$ o polígono $P$ possui tanto para raio de curvatura como função suporte, na transformação $T$, mesmos autovalores. Como estamos trabalhando com polígonos fechados fica justificados o uso de $h$ ao invés de $r$ na transformação $T$, Lema (9.4).

O conjunto $C_{U}$ é invariante pela transformação $T$. Com efeito, lembrando que o conjunto $C_{U}$ é definido de forma que $\sum_{i=1}^{2 n} \Delta_{i} P=0$.

$$
\begin{aligned}
\sum_{i=1}^{2 n} \Delta_{i} P & =\sum_{i=1}^{2 n} r_{i} \Delta_{i} U \\
& =-\sum_{i=1}^{2 n} \Delta_{i} r U_{i+1} \\
& =-\sum_{i=1}^{2 n} \frac{\Delta_{i} r}{\left[V_{i}, V_{i+1}\right]} U_{i+1}\left[V_{i}, V_{i+1}\right]
\end{aligned}
$$




$$
\begin{aligned}
& =-\sum_{i=1}^{2 n} \frac{\Delta_{i} r}{\left[V_{i}, V_{i+1}\right]} \Delta_{i} V \\
& =\sum_{i=1}^{2 n} \nabla_{i}\left(\frac{\Delta_{i} r}{\left[V_{i}, V_{i+1}\right]}\right) V_{i} \\
& =\sum_{i=1}^{2 n} \frac{1}{\left[U_{i}, U_{i+1}\right]} \nabla\left(\frac{\Delta_{i} r}{\left[V_{i}, V_{i+1}\right]}\right) \Delta_{i} U \\
& =\sum_{i=1}^{2 n}\left(-T r_{i}\right) \Delta_{i} U
\end{aligned}
$$

Agora basta substituir $r_{i}$ por $h_{i}(1-\lambda), \lambda \neq 1$ e temos o desejado.

Como T é auto-adjunta e $C_{U}$ é invariante nessa transformação teremos pelo Teorema Espectral que $C_{U}$ possui uma base ortonormal de auto-vetores. Segundo [7] cada elemento da base é a função suporte de uma ciclóide discreta fechada. Assim se $h \in C_{U}$, então $P$ é a soma de $2 n-2$ ciclóides discretas fechadas, que é uma generalização da série de Fourier discreta.

Apresentamos agora de acordo com [7] os autovalores associados a transformação T. Lema (9.4).

$$
\lambda_{0}(=0)<\lambda_{1}^{1}=\lambda_{2}^{1}(=1)<\lambda_{2}^{1} \leq \lambda_{2}^{2}<\lambda_{3}^{1} \leq \lambda_{3}^{2}<\ldots<\lambda_{n-1}^{1} \leq \lambda_{n-1}^{2}<\lambda_{n},
$$

onde $\lambda_{0}(=0)$ indica as ciclóides que são múltiplos da bola, isto é funções suporte constantes, $\lambda_{1}^{1}, \lambda_{2}^{1}$, indicam ciclóides abertas (não incluídas no nosso caso) e cada ciclóide associada a $\lambda_{k}^{j}$ possui exatamente $2 k$ cúspides. Também, se $k$ é par, a ciclóide é simétrica, e se $k$ é ímpar, a ciclóide tem largura zero. Dessa forma se $h \in C_{U}$ teremos:

$$
\begin{gathered}
h=h_{0}+a_{2}^{1} h_{2}^{1}+a_{2}^{2} h_{2}^{2}+a_{3}^{1} h_{3}^{1}+a_{3}^{2} h_{3}^{2}+\ldots+a_{n-1}^{1} h_{n-1}^{1}+a_{n-1}^{2} h_{n-1}^{2}+a_{n} h_{n} \\
=h_{0}+a_{n} h_{n}+\sum_{j=1}^{2} \sum_{k=2}^{n-1} a_{k}^{j} h_{k}^{j}, a_{k}^{j}, a_{n} \in \mathbb{R}, k \geq 2, j=1,2
\end{gathered}
$$

Corolário 9.5. O conjunto $H:=\left\{h_{0}, h_{2}^{1}, h_{2}^{2}, h_{3}^{1}, h_{3}^{2}, \ldots, h_{n-1}^{1}, h_{n-1}^{2}, h_{n}\right\}$ é base ortonormal de $C_{U}$.

Do corolário temos que $\forall h_{1}, h_{2} \in H$

$$
\left\langle h_{1}, h_{2}\right\rangle=A(U), s e, h_{1}=h_{2}
$$




$$
\left\langle h_{1}, h_{2}\right\rangle=0, \text { se, } h_{1} \neq h_{2},
$$

Sabemos também segundo [7] que

$$
\begin{gathered}
h_{k_{i}}^{j}=(-1)^{k} h_{k_{i+n}}^{j}, i \in \mathbb{N}, j=1,2, k=2, \ldots, n-1, \\
h_{n_{i}}=(-1)^{n} h_{n_{i+n}}, n \in \mathbb{N}
\end{gathered}
$$

Teorema 9.6. Sejam $\left\{h_{0}, h_{2}^{1}, h_{2}^{2}, h_{3}^{1}, h_{3}^{2}, \ldots, h_{n-1}^{1}, h_{n-1}^{2}, h_{n}\right\}$ base ortonormal de $C_{U}$ e $F_{k}^{j}$ ciclóide associada à função suporte $h_{k}^{j}$. Então

1. $C W\left(F_{k}^{j}\right)=F_{k}^{j}$ e $C M L C\left(F_{k}^{j}\right)=\{0\}, k$ impar.

2. $C W\left(F_{k}^{j}\right)=\{0\}$ e $C M L C\left(F_{k}^{j}\right)=F_{k}^{j}, k$ par.

Demonstração. Para $k$ ímpar a equação (9.6) e a proposição (7.6) implicam que $F_{k_{i}}^{j}=F_{k_{i+n}}^{j}$, então $C W\left(F_{k_{i}}^{j}\right)=F_{k_{i}}^{j}$. Pela proposição (7.16) $C M L C\left(F_{k_{i}}^{j}\right)=$ $\{0\}$. Para $k$ par a equação (9.6) e a proposição (7.9) mostram que $F_{k}^{j}$ é simétrica e portanto $C W\left(F_{k}^{j}\right)=\{0\}$. Pela equação (9.5) temos

$$
\begin{aligned}
L_{V}\left(F_{k_{i}}^{j}\right) & =\sum_{i=1}^{2 n} r_{F_{k_{i}}^{j}}\left[U_{i}, U_{i+1}\right] \\
& =\sum_{i=1}^{2 n} h_{k_{i}}^{j}\left(1-\lambda_{k}^{j}\right)\left[U_{i}, U_{i+1}\right]=0
\end{aligned}
$$

Portanto $\bar{w}_{F_{k}^{j}}=\frac{L_{V}\left(F_{k}^{j}\right)}{A(u)}=0$ e simetria implicam $C M L C\left(F_{k}^{j}\right)=F_{k}^{j}$

\section{1}

\section{Áreas e Comprimentos}

Nessa seção vamos apresentar o cálculo de áreas orientadas e comprimento dual de polígonos em $C_{U}$ estudados até o momento, utilizando a base ortonormal de $C_{U}$, corolário (9.5), isto é, a série de Fourier generalizada discreta.

$$
\begin{aligned}
2 \widetilde{\mathrm{A}}(C W(P)) & =\frac{1}{2} \sum_{i=1}^{2 n}\left[C W\left(P_{i}\right), \Delta_{i} C W(P)\right] \\
& =\frac{1}{2} \sum_{i=1}^{2 n}\left[C W\left(P_{i}\right), C W\left(P_{i+1}\right)\right]
\end{aligned}
$$




$$
\begin{aligned}
& =\frac{1}{2} \sum_{i=1}^{2 n}\left(h_{C W\left(P_{i}\right)}\right)^{2}\left[U_{i}, U_{i+1}\right]-\frac{\left(\Delta_{i} h_{C W(P)}\right)^{2}}{\left[V_{i}, V_{i+1}\right]} \\
& =\frac{1}{2} \sum_{i=1}^{2 n}\left(\frac{h_{i}-h_{i+n}}{2}\right)^{2}\left[U_{i}, U_{i+1}\right]-\frac{\left(\Delta_{i} h-\Delta_{i+n} h\right)^{2}}{\left[V_{i}, V_{i+1}\right]} \\
& =\frac{1}{2} A(P)-\frac{1}{4} \sum_{i=1}^{2 n} h_{i} h_{i+n}\left[U_{i}, U_{i+1}\right]-\frac{\left(\Delta_{i} h\right)\left(\Delta_{i+n} h\right)}{\left[V_{i}, V_{i+1}\right]} \\
& =\frac{1}{2} A(P)-\frac{1}{4} \sum_{i=1}^{2 n}\left(h_{0_{i}}+a_{n} h_{n_{i}}+\sum_{\substack{k \geqslant 2 \\
j=1,2}}^{n-1} a_{k}^{j} h_{k_{i}}^{j}\right)\left(h_{0_{i+n}}+a_{n} h_{n_{i+n}}+\sum_{\substack{k \geqslant 2 \\
j=1,2}}^{n-1} a_{k}^{j} h_{k_{i+n}}^{j}\right)\left[U_{i}, U_{i+1}\right] \\
& -\frac{1}{\left[V_{i}, V_{i+1}\right]}\left(a_{n} \Delta_{i} h_{n}+\sum_{\substack{k \geqslant 2 \\
j=1,2}}^{n-1} a_{k}^{j} \Delta_{i} h_{k}^{j}\right)\left(a_{n} \Delta_{i+n} h_{n}+\sum_{\substack{k \geqslant 2 \\
j=1,2}}^{n-1} a_{k}^{j} \Delta_{i+n} h_{k}^{j}\right) \\
& =\frac{A(P)}{2}-\frac{1}{4} \sum_{i=1}^{2 n} h_{0}^{2}\left[U_{i}, U_{i+1}\right]-\frac{1}{4}\left(a_{n}\right)^{2}(-1)^{n} \sum_{i=1}^{2 n}\left(h_{n_{i}}\right)^{2}\left[U_{i}, U_{i+1}\right] \\
& -\frac{1}{4} \sum_{\substack{k \geqslant 2 \\
j=1,2}}^{n-1}\left(a_{k}^{j}\right)^{2}(-1)^{k} \sum_{i=1}^{2 n}\left(h_{k_{i}}^{j}\right)^{2}\left[U_{i}, U_{i+1}\right]+\frac{1}{4}(-1)^{n}\left(a_{n}\right)^{2} \sum_{i=1}^{2 n} \frac{\left(\Delta_{i} h_{n}\right)^{2}}{\left[V_{i}, V_{i+1}\right]} \\
& +\frac{1}{4} \sum_{\substack{k \geqslant 2 \\
j=1,2}}^{n-1}(-1)^{k}\left(a_{k}^{j}\right)^{2} \sum_{i=1}^{2 n} \frac{\left(\Delta_{i} h_{k}^{j}\right)^{2}}{\left[V_{i}, V_{i+1}\right]} \\
& =\frac{A(P)}{2}-\frac{2 A(U)}{4} h_{0}^{2}-\frac{A(U)}{4}(-1)^{n}\left(a_{n}\right)^{2}-\frac{A(U)}{4} \sum_{\substack{k \geqslant 2 \\
j=1,2}}^{n-1}(-1)^{k}\left(a_{k}^{j}\right)^{2} \\
& +\frac{A(U)}{4}\left(a_{n}\right)^{2}(-1)^{n} \lambda_{n}+\frac{A(U)}{4} \sum_{\substack{k \geqslant 2 \\
j=1,2}}^{n-1}(-1)^{k}\left(a_{k}^{j}\right)^{2} \lambda_{k}^{j} \\
& =\frac{A(P)}{2}-\frac{2 A(U)}{4} h_{0}^{2}+\frac{A(U)}{4}(-1)^{n}\left(a_{n}\right)^{2}\left(\lambda_{n}-1\right)+\frac{A(U)}{4} \sum_{\substack{k \geqslant 2 \\
j=1,2}}^{n-1}(-1)^{k}\left(a_{k}^{j}\right)^{2}\left(\lambda_{k}^{j}-1\right) \\
& =\frac{h_{0}^{2} A(U)}{2}-\frac{A(U)}{4}\left(a_{n}\right)^{2}\left(\lambda_{n}-1\right)-\frac{A(U)}{4} \sum_{\substack{k \geqslant 2 \\
j=1,2}}^{n-1}\left(a_{k}^{j}\right)^{2}\left(\lambda_{k}^{j}-1\right) \\
& -\frac{A(U)}{2} h_{0}^{2}+\frac{A(U)}{4}(-1)^{n}\left(a_{n}\right)^{2}\left(\lambda_{n}-1\right)+\frac{A(U)}{4} \sum_{\substack{k \geqslant 2 \\
j=1,2}}^{n-1}(-1)^{k}\left(a_{k}^{j}\right)^{2}\left(\lambda_{k}^{j}-1\right) \\
& =-\frac{A(U)}{4}\left(\left(a_{n}\right)^{2}\left(\lambda_{n}-1\right)\left(1-(-1)^{n}\right)+\sum_{\substack{k=i \text { mpar } \\
j=1,2}}^{n-1} 2\left(a_{k}^{j}\right)^{2}\left(\lambda_{k}^{j}-1\right)\right) \\
& =-\frac{A(U)}{2}\left(\frac{\left(a_{n}\right)^{2}\left(\lambda_{n}-1\right)\left(1-(-1)^{n}\right)}{2}+\sum_{\substack{k=\text { impar } \\
j=1,2}}^{n-1}\left(a_{k}^{j}\right)^{2}\left(\lambda_{k}^{j}-1\right)\right)
\end{aligned}
$$




$$
\begin{aligned}
& \widetilde{\mathrm{A}}_{C M L C(P)}=\frac{1}{2} \sum_{i=1}^{2 n}\left[C M L C\left(P_{i}\right), \Delta_{i} C M L C(P)\right] \\
& =\frac{1}{2} \sum_{i=1}^{2 n}\left[C M L C\left(P_{i}\right), C M L C\left(P_{i+1}\right)\right] \\
& =\frac{1}{2} \sum_{i=1}^{2 n}\left(h_{C M L C\left(P_{i}\right)}\right)^{2}\left[U_{i}, U_{i+1}\right]-\frac{\left(\Delta_{i} h_{C M L C(P)}\right)^{2}}{\left[V_{i}, V_{i+1}\right]} \\
& =\frac{1}{8} \sum_{i=1}^{2 n}\left(h_{i}+h_{i+n}-\bar{w}_{P}\right)^{2}\left[U_{i}, U_{i+1}\right]-\frac{\left(\Delta_{i} h+\Delta_{i+n} h\right)^{2}}{\left[V_{i}, V_{i+1}\right]} \\
& =\frac{1}{4}\left(2 A(P)-4 h_{0} A(U) \bar{w}_{P}+\left(\bar{w}_{P}\right)^{2} A(U)+\sum_{i=1}^{2 n} h_{i} h_{i+n}\left[U_{i}, U_{i+1}\right]-\frac{\left(\Delta_{i} h\right)\left(\Delta_{i+n} h\right)}{\left[V_{i}, V_{i+1}\right]}\right) \\
& =\frac{1}{4}\left(2 A(P)-4 h_{0} A(U) \bar{w}_{P}+\left(\bar{w}_{P}\right)^{2} A(U)+2 A(U) h_{0}^{2}-A(U)(-1)^{n}\left(a_{n}\right)^{2}\left(\lambda_{n}-1\right)\right. \\
& \left.-A(U) \sum_{\substack{k \geqslant 2 \\
j=1,2}}^{n-1}(-1)^{k}\left(a_{k}^{j}\right)^{2}\left(\lambda_{k}^{j}-1\right)\right) \\
& =\frac{1}{2}\left(h_{0}^{2} A(U)-\frac{A(U)}{2}\left(a_{n}\right)^{2}\left(\lambda_{n}-1\right)-\frac{A(U)}{2} \sum_{\substack{k \geqslant 2 \\
j=1,2}}^{n-1}\left(a_{k}^{j}\right)^{2}\left(\lambda_{k}^{j}-1\right)\right)-h_{0}^{2} A(U) \\
& +\frac{1}{2} A(U) h_{0}^{2}-\frac{A(U)}{4}(-1)^{n}\left(a_{n}\right)^{2}\left(\lambda_{n}-1\right)-\frac{A(U)}{4} \sum_{\substack{k \geqslant 2 \\
j=1,2}}^{n-1}(-1)^{k}\left(a_{k}^{j}\right)^{2}\left(\lambda_{k}^{j}-1\right) \\
& =-\frac{A(U)}{2}\left(\frac{\left(a_{n}\right)^{2}\left(\lambda_{n}-1\right)\left(1+(-1)^{n}\right)}{2}+\sum_{\substack{k=p a r \\
j=1,2}}^{n-1}\left(a_{k}^{j}\right)^{2}\left(\lambda_{k}^{j}-1\right)\right) \\
& L_{V}(P)=\sum_{i=1}^{2 n}\left\|\Delta_{i} P\right\|_{V} \\
& =\sum_{i=1}^{2 n}\left(h_{i}\left[U_{i}, U_{i+1}\right]+\nabla_{i}\left(\frac{\Delta_{i} h}{\left[V_{i}, V_{i+1}\right]}\right)\right)\left\|V_{i}\right\| \\
& =\sum_{i=1}^{2 n} h_{i}\left[U_{i}, U_{i+1}\right] \\
& =\sum_{i=1}^{2 n}\left(h_{0_{i}}+a_{n} h_{n_{i}}+\sum_{\substack{k \geqslant 2 \\
j=1,2}}^{n-1} a_{k}^{j} h_{k_{i}}^{j}\right)\left[U_{i}, U_{i+1}\right] \\
& =h_{0} \sum_{i=1}^{2 n}\left[U_{i}, U_{i+1}\right]+a_{n} \sum_{i=1}^{2 n} h_{n_{i}}\left[U_{i}, U_{i+1}\right]+\sum_{\substack{k \geqslant 2 \\
j=1,2}}^{n-1} a_{k}^{j} \sum_{i=1}^{2 n} h_{k_{i}}^{j}\left[U_{i}, U_{i+1}\right] \\
& =2 h_{0} A(U)
\end{aligned}
$$




$$
\begin{aligned}
& \widetilde{\mathrm{A}}_{P}=\frac{1}{2} \sum_{i=1}^{2 n}\left[P_{i}, P_{i-1}-P_{i}\right] \\
& =\frac{1}{2} \sum_{i=1}^{2 n}\left[P_{i}, \Delta_{i} P\right] \\
& =\frac{1}{2} \sum_{i=1}^{2 n}\left[h_{i} U_{i}+\left(\Delta_{i-1} h\right) \frac{V_{i}}{\left[V_{i-1}, V_{i}\right]},\left(h_{i}\left[U_{i}, U_{i+1}\right]+\nabla_{i}\left(\frac{\Delta_{i} h}{\left[V_{i}, V_{i+1}\right]}\right)\right) V_{i}\right] \\
& =\frac{1}{2} \sum_{i=1}^{2 n}\left(h_{i}\right)^{2}\left[U_{i}, U_{i+1}\right]+h_{i} \nabla_{i}\left(\frac{\Delta_{i} h}{\left[V_{i}, V_{i+1}\right]}\right) \\
& =\frac{1}{2} \sum_{i=1}^{2 n}\left(h_{i}\right)^{2}\left[U_{i}, U_{i+1}\right]-\left(\frac{\left(\Delta_{i} h\right)\left(\Delta_{i} h\right)}{\left[V_{i}, V_{i+1}\right]}\right) \\
& =\frac{1}{2} \sum_{i=1}^{2 n}\left(h_{i}\right)^{2}\left[U_{i}, U_{i+1}\right]-\frac{\left(\Delta_{i} h\right)^{2}}{\left[V_{i}, V_{i+1}\right]} \\
& =\frac{1}{2} \sum_{i=1}^{2 n}\left(h_{0_{i}}+a_{n} h_{n_{i}}+\sum_{\substack{k \geqslant 2 \\
j=1,2}}^{n-1} a_{k}^{j} h_{k_{i}}^{j}\right)^{2}\left[U_{i}, U_{i+1}\right]-\frac{\left(a_{n} \Delta_{i} h_{n}+\sum_{\substack{k \geqslant 2 \\
j=1,2}}^{n-1} a_{k}^{j} \Delta_{i} h_{k}^{j}\right)^{2}}{\left[V_{i}, V_{i+1}\right]} \\
& =h_{0}^{2} \frac{1}{2} \sum_{i=1}^{2 n}\left[U_{i}, U_{i+1}\right]+\frac{1}{2}\left(a_{n}\right)^{2} \sum_{i=1}^{2 n}\left(h_{n_{i}}\right)^{2}\left[U_{i}, U_{i+1}\right]+\frac{1}{2} \sum_{\substack{k \geqslant 2 \\
j=1,2}}^{n-1}\left(a_{k}^{j}\right)^{2} \sum_{i=1}^{2 n}\left(h_{k_{i}}\right)^{2}\left[U_{i}, U_{i+1}\right] \\
& -\frac{1}{2} \sum_{i=1}^{2 n}\left(a_{n}\right)^{2} \frac{\left(\Delta_{i} h_{n}\right)^{2}}{\left[V_{i}, V_{i+1}\right]}-\frac{1}{2} \sum_{\substack{k \geqslant 2 \\
j=1,2}}^{n-1}\left(a_{k}^{j}\right)^{2} \sum_{i=1}^{2 n} \frac{\left(\Delta_{i} h_{k}^{j}\right)^{2}}{\left[V_{i}, V_{i+1}\right]} \\
& =h_{0}^{2} A(U)+\frac{1}{2}\left(a_{n}\right)^{2} A(U)+\frac{1}{2} A(U) \sum_{\substack{k \geqslant 2 \\
j=1,2}}^{n-1}\left(a_{k}^{j}\right)^{2}-\frac{1}{2}\left(a_{n}\right)^{2} \lambda_{n} A(U)-\frac{1}{2} \sum_{\substack{k \geqslant 2 \\
j=1,2}}^{n-1}\left(a_{k}^{j}\right)^{2} \lambda_{k}^{j} A(U) \\
& =h_{0}^{2} A(U)+\frac{A(U)}{2} a_{n}^{2}\left(1-\lambda_{n}\right)+\frac{A(U)}{2} \sum_{\substack{k \geqslant 2 \\
j=1,2}}^{n-1}\left(a_{k}^{j}\right)^{2}\left(1-\lambda_{k}^{j}\right) .
\end{aligned}
$$

Corolário 9.7. Todo polígono $P \in C_{U}$ com $V$-comprimento zero, isto é, $h_{0}=0$ possui $\widetilde{\mathrm{A}}_{P}<0$. Em particular a proposição (7.27) está demonstrada. 


\section{2}

\section{Outra Prova - Igualdade Isoperimétrica em Plano Normado - Discreto}

Teorema 9.8. Seja $P=\left\{P_{1}, \ldots, P_{2 n}\right\}$ polígono em $C_{U}$ no plano $\left(\mathbb{R}^{2}, U\right)$, onde $U=\left\{U_{1}, \ldots, U_{2 n}\right\}$ é a bola unitária e $P_{i+1}-P_{i}$ é paralelo a $U_{i+1}-U_{i}$. Então

$$
L_{V}^{2}(P)=4 A_{U} \widetilde{\mathrm{A}}_{P}-8 A_{U} \widetilde{\mathrm{A}}_{C W(P)}-4 A_{U} \widetilde{\mathrm{A}}_{C M L C(P)},
$$

onde $L_{V}(P)$ indica o $V$-comprimento com sinal do polígono $P, A_{U}$ indica área da bola unitária e $\widetilde{\mathrm{A}}(P), \widetilde{\mathrm{A}}_{C W(P)}$ e $\widetilde{\mathrm{A}}_{C M L C(P)}$ indicam respectivamente as áreas com sinal de $P, C W(P)$ e $C M L C(P)$.

Demonstração. Da seção anterior temos

$$
\begin{aligned}
& L_{V}(P)=2 h_{0} A_{U} \\
& \tilde{\mathrm{A}}_{P}=h_{0}^{2} A_{U}-\frac{A_{U}}{2}\left(a_{n}\right)^{2}\left(\lambda_{n}-1\right)-\frac{A_{U}}{2} \sum_{\substack{k \geqslant 2 \\
j=1,2}}^{n-1}\left(a_{k}^{j}\right)^{2}\left(\lambda_{k}^{j}-1\right) \\
& \tilde{\mathrm{A}}_{C W(P)}=-\frac{A_{U}}{4}\left(\frac{\left(a_{n}\right)^{2}\left(\lambda_{n}-1\right)\left(1-(-1)^{n}\right)}{2}+\sum_{\substack{k=1 \text { mpar } \\
j=1,2}}^{n-1}\left(a_{k}^{j}\right)^{2}\left(\lambda_{k}^{j}-1\right)\right) \\
& \widetilde{\mathrm{A}}_{C M L C(P)}=-\frac{A_{U}}{2}\left(\frac{\left(a_{n}\right)^{2}\left(\lambda_{n}-1\right)\left(1+(-1)^{n}\right)}{2}+\sum_{\substack{k=p a r \\
j=1,2}}^{n-1}\left(a_{k}^{j}\right)^{2}\left(\lambda_{k}^{j}-1\right)\right)
\end{aligned}
$$

Observe que $\widetilde{\mathrm{A}}_{C W(P)} \leq 0$ e $\widetilde{\mathrm{A}}_{C W M S(P)} \leq 0$ pois $\lambda_{k}^{j}>1$ (ver [7]). Substituindo.

$$
4 A_{U} \tilde{\mathrm{A}}_{P}-8 A_{U} \tilde{\mathrm{A}}_{C W(P)}-4 A_{U} \tilde{\mathrm{A}}_{C M L C(P)}=
$$




$$
\begin{aligned}
& 4 A_{U}\left(h_{0}^{2} A_{U}-\frac{A_{U}}{2}\left(a_{n}\right)^{2}\left(\lambda_{n}-1\right)-\frac{A_{U}}{2} \sum_{\substack{k \geqslant 2 \\
j=1,2}}^{n-1}\left(a_{k}^{j}\right)^{2}\left(\lambda_{k}^{j}-1\right)\right) \\
& +8 A_{U} \frac{A_{U}}{4}\left(\frac{\left(a_{n}\right)^{2}\left(\lambda_{n}-1\right)\left(1-(-1)^{n}\right)}{2}+\sum_{\substack{k=i \text { mpar } \\
j=1,2}}^{n-1}\left(a_{k}^{j}\right)^{2}\left(\lambda_{k}^{j}-1\right)\right) \\
& +4 A_{U} \frac{A_{U}}{2}\left(\frac{\left(a_{n}\right)^{2}\left(\lambda_{n}-1\right)\left(1+(-1)^{n}\right)}{2}+\sum_{\substack{k=\text { par } \\
j=1,2}}^{n-1}\left(a_{k}^{j}\right)^{2}\left(\lambda_{k}^{j}-1\right)\right) \\
& =4 h_{0}^{2}\left(A_{U}\right)^{2}=\left(2 h_{0} A_{U}\right)^{2}=L_{V}^{2}(P)
\end{aligned}
$$


10

\section{Curvas Rosáceas}

Uma $m$-rosácea é uma curva suave fechada com curvatura não-nula e número de rotações igual a $m$ onde $m$ é um inteiro positivo(ver [3], [15]). O conjunto das $m$-rosáceas é representado por $\mathscr{H}_{m}$, veja definição (2.8).

Observe que pela definição (2.8) as curvas $m$-rosácea são $2 m \pi$-periódicas. Por [6], teorema (4.4), utilizando uma norma qualquer do plano podemos calcular os autovalores $\lambda_{k, m}^{j}$ da equação (5.1) e seus respectivos autovetores $h_{k, m}^{j}$ (ciclóides) para curvas fechadas com $m$ rotações, isto é, $m$-rosácea. No caso euclidiano, por exemplo, a função suporte $h(\theta)$ da $m$-rosácea pode ser escrita utilizando Fourier como

$$
h(\theta)=h_{0}+\sum_{k=1}^{\infty} a_{n} \cos \left(\frac{k \theta}{m}\right)+b_{n} \sin \left(\frac{k \theta}{m}\right), \theta \in[0,2 m \pi] .
$$

\section{1}

\section{Exemplo}

Exemplo 10.1. Considere $u(\theta)=(\cos (\theta), \sin (\theta))$ como bola unitária, $h(\theta)=$ $\cos \left(\frac{\theta}{2}\right)+2$ função suporte de uma 2 -rosácea. Seja $\gamma(\theta)$ uma parametrização de 2-rosácea. Utilizando a equação

$$
\gamma(\theta)=h(\theta) u(\theta)+\frac{h^{\prime}(\theta)}{\left[v(\theta), v^{\prime}(\theta)\right]} v(\theta)
$$

teremos

$$
\begin{aligned}
\gamma(\theta) & =(\cos (t / 2) \cos (t)+(\sin (t / 2) \sin (t)) / 2+2 \cos (t), \cos (t / 2) \sin (t) \\
& -(\sin (t / 2) \cos (t)) / 2+2 \sin (t))
\end{aligned}
$$




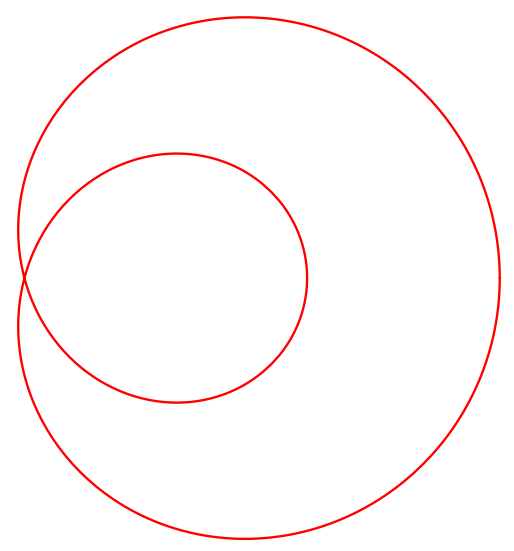

Figura 10.1: 2-rosácea

De acordo com [25] para cada $m$-rosácea podemos calcular $\frac{(m+1) m}{2}$ "ramos"de Cáusticas de Wigner. Nós vamos nos deter a apenas um dentre esses "ramos"de Cáusticas de Wigner, pois a partir dele obtemos a igualdade isoperimétrica. Para obter "ramos"de Cáustica de Wigner basta variar $k=$ $1, \ldots, m$ da definição $C W(\theta)=(\gamma(\theta)+\gamma(\theta+k \pi)) / 2$. Vamos nos deter no caso $k=m$.

\section{2}

\section{Cáustica de Wigner}

Definição 10.2. Seja $\gamma \in \mathscr{H}_{m}$. Chamamos Evoluta de Área ou Cáustica de Wigner $(C W) \gamma$ o conjunto

$$
C W(\gamma)(\theta)=\{(\gamma(\theta)+\gamma(\theta+m \pi)) / 2 ; \theta \in[0,2 m \pi]\}
$$

Como $C W(\gamma)(\theta)=C W(\gamma)(\theta+m \pi)$ temos que $C W(\gamma)(\theta)$ possui período $m \pi$ e dá duas voltas no intervalo $[0,2 m \pi]$. Seus cúspides ocorrem quando $r(\theta)=\left((-1)^{m+1}\right) r(\theta+m \pi)$, pois $C W^{\prime}(\gamma)(\theta)=\left(\frac{r(\theta)+(-1)^{m} r(\theta+m \pi)}{2}\right) u^{\prime}(\theta)$. A função suporte de $C W(\gamma)(\theta)$ é

$$
\left.h_{C W(\gamma)}=[\gamma(\theta)+\gamma(\theta+m \pi)) / 2, v(\theta)\right]=\frac{1}{2}\left(h(\theta)+(-1)^{m} h(\theta+m \pi)\right) .
$$

Onde $h(\theta)$ é a função suporte de $\gamma$. 


\section{3}

\section{Conjunto de Medida de Largura Constante}

Definição 10.3. Seja $\gamma \in \mathscr{H}_{m}$. Definimos como Conjunto de Medida de Largura constante de $\gamma$, abreviado por $C M L C(\gamma)$,

$$
C M L C(\gamma)(\theta)=\left\{\frac{1}{2}\left(\gamma(\theta)-\gamma(\theta+m \pi)-\frac{\bar{w}_{\gamma}}{m} u(\theta)\right), \theta \in[0,2 m \pi]\right\}
$$

Ver [25] para versão Euclidiana do CMLC.

A função suporte de $C M L C$ é

$$
\begin{aligned}
h_{C M L C(\gamma)}(\theta) & =\frac{1}{2}\left[\gamma(\theta)-\gamma(\theta+m \pi)-\frac{\bar{w}_{\gamma}}{m} u(\theta), v(\theta)\right] \\
& =\frac{1}{2}\left(h(\theta)-(-1)^{m} h(\theta+m \pi)-\frac{\bar{w}_{\gamma}}{m}\right) .
\end{aligned}
$$

10.4

Decomposição da curva $\gamma \in \mathscr{H}_{m}$ como soma de Caústica de Wigner, CMLC e bola unitária.

Proposição 10.4. $\gamma \in \mathscr{H}_{m} \cdot \gamma(\theta)=C W(\gamma)(\theta)+C M L C(\gamma)(\theta)+\frac{\bar{w}_{\gamma}}{2 m} u(\theta) e$ $h_{\gamma}(\theta)=h_{C W(\gamma)}(\theta)+h_{C M L C(\gamma)}(\theta)+\frac{\bar{w}_{\gamma}}{2 m}$.

Corolário 10.5. $\gamma \in \mathscr{H}_{m} \cdot \gamma(\theta)=C W(\gamma)(\theta)+C M L C(\gamma)(\theta)$ e $h_{\gamma}(\theta)=$ $h_{C W(\gamma)}(\theta)+h_{C M L C(\gamma)}(\theta) \Leftrightarrow L_{v}(\gamma)=0$

É simples ver que os resultados acima são consequência do fato que

$$
\gamma(\theta)=\frac{\gamma(\theta)+\gamma(\theta+m \pi)}{2}+\frac{\gamma(\theta)-\gamma(\theta+m \pi)}{2} .
$$

Pela proposição (10.5) podemos reescrever $\gamma$ utilizando bases ortonormais. Com efeito

$$
\begin{aligned}
\gamma(\theta) & =h_{C W}(\theta) u(\theta)+\frac{\left(h_{C W}\right)^{\prime}(\theta)}{\left[v(\theta), v^{\prime}(\theta)\right]} v(\theta) \\
& +h_{C M L C}(\theta) u(\theta)+\frac{\left(h_{C M L C}\right)^{\prime}(\theta)}{\left[v(\theta), v^{\prime}(\theta)\right]} v(\theta)+\frac{\bar{w}_{\gamma}}{2 m} u(\theta)
\end{aligned}
$$




$$
\begin{aligned}
& \text { mpar } \Rightarrow \gamma(\theta)=\left(h_{0}+\sum_{\substack{k \text { par } \\
j=1,2}}^{\infty} a_{k}^{j} h_{k, m}^{j}(\theta)\right) u(\theta)+\frac{\sum_{k p a r}^{\infty} a_{k}^{j}\left(h_{k, m}^{j}\right)^{\prime}(\theta)}{\left[v(\theta), v^{\prime}(\theta)\right]} v(\theta) \\
& +\left(\sum_{\substack{k \text { impar } \\
j=1,2}}^{\infty} a_{k}^{j} h_{k, m}^{j}(\theta)-h_{0}\right) u(\theta)+\frac{\sum_{k \text { ímpar }}^{\infty} a_{k}^{j}\left(h_{k, m}^{j}\right)^{\prime}(\theta)}{\left[v(\theta), v^{\prime}(\theta)\right]} v(\theta) \\
& +\frac{\bar{w}_{\gamma}}{2 m} u(\theta) .
\end{aligned}
$$

$$
\begin{aligned}
& \text { mímpar } \Rightarrow \gamma(\theta)=\left(\sum_{\substack{\text { impar } \\
j=1,2}}^{\infty} a_{k}^{j} h_{k, m}^{j}(\theta)\right) u(\theta)+\frac{\sum_{k i m p a r}^{\infty} a_{k}^{j}\left(h_{k, m}^{j}\right)^{\prime}(\theta)}{\left[v(\theta), v^{\prime}(\theta)\right]} v(\theta) \\
& +\left(\sum_{\substack{k \text { par } \\
j=1,2}}^{\infty} a_{k}^{j} h_{k, m}^{j}(\theta)\right) u(\theta)+\frac{\sum_{k p a r}^{\infty} a_{k}^{j}\left(h_{k, m}^{j}\right)^{\prime}(\theta)}{\left[v(\theta), v^{\prime}(\theta)\right]} v(\theta) \\
& +\frac{\bar{w}_{\gamma}}{2 m} u(\theta) .
\end{aligned}
$$

\section{5}

\section{Cuspides CMLC e Cáustica de Wigner}

Teorema 10.6. Seja $\gamma \in \mathscr{H}_{m}$ curva que possui apenas singularidades ordinárias, isto é, do tipo 2-cúspide. Se $m$ é ímpar então o número de 2 -cúspides de $C M L C(\gamma)$ no intervalo $[0,2 m \pi]$ é múltiplo de quatro. Se $m$ é par e $\bar{w}_{\gamma}=0$ então o número de 2-cúspides de $C M L C(\gamma)$ no intervalo $[0,2 m \pi]$ é $2 k$ com $k$ impar.

Demonstração. O número de cúspides de $C M L C$ é igual ao número de zeros de $r_{C M L C(\gamma)}(\theta)=\frac{1}{2}\left(r(\theta)-(-1)^{m} r(\theta+m \pi)-\frac{\bar{w}_{\gamma}}{m}\right)$ no intervalo de 0 até $2 m \pi$. Para m ímpar $r_{C M L C(\gamma)}(\theta)=r_{C M L C(\gamma)}(\theta+m \pi)$ então o número de cúspides de $C M L C(\gamma)$ de 0 a $m \pi$ é par, como $r_{C M L C(\gamma)}(\theta)$ é $m \pi$-periódica para m ímpar concluímos que o números de cúspides deve ser múltiplo de quatro. Para $\mathrm{m}$ par $r_{C M L C(\gamma)}(\theta)=\frac{1}{2}\left(r(\theta)-r(\theta+m \pi)-\frac{\bar{w}_{\gamma}}{m}\right)$ como por hipótese $\bar{w}_{\gamma}=0$ implica $r_{C M L C(\gamma)}(\theta+m \pi)=-r_{C M L C(\gamma)}(\theta)$ então o número de zeros deve ser ímpar em $[0, m \pi]$ e em $[m \pi, 2 m \pi]$. 
Teorema 10.7. Seja $\gamma \in \mathscr{H}_{m}$ curva que possui apenas singularidades ordinárias, isto é, do tipo 2-cúspide. Se m é par então o número de 2-cúspides de $C W(\gamma)$ no intervalo $[0,2 m \pi]$ é múltiplo de quatro. Se $m$ é ímpar então o

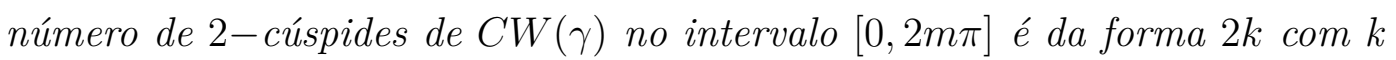
impar.

Demonstração. Observe que $C W^{\prime}(\gamma)(\theta)=\frac{r(\theta)+(-1)^{m} r(\theta+m \pi)}{2} u^{\prime}(\theta)$. Assim, $r_{C W(\gamma)}(\theta)=\frac{r(\theta)+(-1)^{m} r(\theta+m \pi)}{2}$. Se $m$ é par então $r_{C W(\gamma)}(\theta)=$ $r_{C W(\gamma)}(\theta+m \pi)$ então o número de zeros de $r_{C W(\gamma)}$ de $\theta$ até $\theta+m \pi$ é par. Se $m$ é impar então $r_{C W(\gamma)}(\theta)=-r_{C W(\gamma)}(\theta+m \pi)$ então o número de zeros de $\theta$ até $\theta+m \pi$ é ímpar. Temos o desejado.

Destacamos que os dois resultados anteriores são válidos para 2 -cúspides, isto é, se $\gamma \in \mathscr{H}_{m}$ é tal que $\gamma^{\prime}(\alpha)=0$ então $r(\alpha)=0$ e $r^{\prime}(\alpha) \neq 0$ onde $r$ é o raio de curvatura.

No teorema 10.6 temos a condição $\bar{w}_{\gamma}=0$ para $m$ par. Como contra exemplo considere o $C M L C$ de função suporte $h_{C M L C}(\theta)=5 \cos (3 \theta / 4)-$ $\cos (\theta / 4)-2$ e considere como bola unitária o círculo Euclidiano. Neste contra exemplo $m=4, \bar{w}_{\gamma}=16$ e o número de 2 -cúspides é 4 .

\section{6 \\ Exemplo}

Exemplo 10.8. Considere $u(\theta)=(\cos (\theta), \sin (\theta))$ como bola unitária, $h(\theta)=$ $\cos \left(\frac{\theta}{2}\right)+2$ função suporte de uma 2 -rosácea. Seja $\gamma(\theta)$ uma parametrização de 2-rosácea. Podemos calcular $C W(\gamma)$ e $C M L C(\gamma)$.

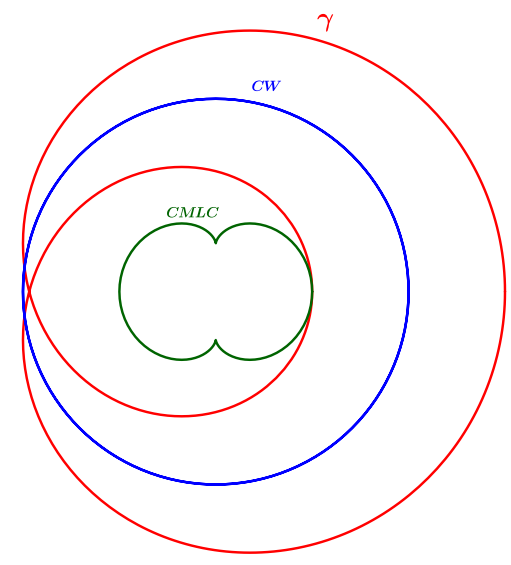

Figura 10.2: 2-rosácea, CW, CMLC 


\section{7}

\section{Igualdade Isoperimétrica em Plano Normado para $m$-rosáceas}

Teorema 10.9 (Igualdade Isoperimétrica em Plano Normado para curvas $m$-rosáceas). Seja $\gamma$ uma $m$-rosáceas em $\mathscr{H}_{m}$ no plano $\left(\mathbb{R}^{2}, U\right)$, onde $U$ é a bola unitária. Então

I) Se m é impar então

$$
L_{v}^{2}(\gamma)=4 m A_{U} \widetilde{\mathrm{A}}_{\gamma}-8 m A_{U} \widetilde{\mathrm{A}}_{C W(\gamma)}-4 m A_{U} \widetilde{\mathrm{A}}_{C M L C(\gamma)} .
$$

II) Se m é par então

$$
L_{v}^{2}(\gamma)=-4 m A_{U} \tilde{\mathrm{A}}_{\gamma}+8 m A_{U} \widetilde{\mathrm{A}}_{C W(\gamma)}+4 m A_{U} \widetilde{\mathrm{A}}_{C M L C(\gamma)} .
$$

onde $L_{v}(\gamma)$ indica o comprimento com sinal da curva $\gamma$ na norma dual, $A_{U}$ a área da bola unitária, $\widetilde{\mathrm{A}}_{\gamma}, \widetilde{\mathrm{A}}_{C W(\gamma)}$ e $\widetilde{\mathrm{A}}_{C M L C(\gamma)}$ indicam respectivamente as áreas com sinal de $\gamma, C W(\gamma)$ e $C M L C(\gamma)$.

\section{Demonstração.}

Pela proposição (10.4) temos

$$
\gamma(\theta)=C W(\gamma)(\theta)+C M L C(\gamma)(\theta)+\frac{\bar{w}_{\gamma}}{2 m} u(\theta)
$$

Vamos proceder da seguinte maneira: calcular a área de $\gamma(\theta)$ utilizando a proposição (10.4) e assim obter a Igualdade Isoperimétrica. Iremos também utilizar um resultado de integração por partes

$$
\int_{0}^{2 m \pi}\left[f^{\prime}(t), g(t)\right] d t=-\int_{0}^{2 m \pi}\left[f(t), g^{\prime}(t)\right] d t
$$

onde $f, g$ são de classe $C^{1}$ e que

$$
\int_{0}^{2 m \pi}\left[C M L C(\gamma)(\theta), C W^{\prime}(\gamma)(\theta)\right] d \theta=-\frac{L_{v}^{2}(\gamma)}{4 m A_{U}}\left(1+(-1)^{m}\right)
$$

e

$$
\int_{0}^{2}\left[u(\theta), C W^{\prime}(\theta)+C M L C^{\prime}(\theta)\right] d \theta=0 .
$$




$$
\begin{aligned}
\tilde{\mathrm{A}}(\gamma) & =\frac{1}{2} \int_{0}^{2 m \pi}\left[\gamma(\theta), \gamma^{\prime}(\theta)\right] d \theta \\
& =\frac{1}{2} \int_{0}^{2 m \pi}\left[C W(\theta)+C M L C(\theta)+\frac{\bar{w}_{\gamma}}{2 m} u(\theta), C W^{\prime}(\theta)+C M L C^{\prime}(\theta)+\frac{\bar{w}_{\gamma}}{2 m} u^{\prime}(\theta)\right] d \theta \\
& =\frac{1}{2} \int_{0}^{2 m \pi}\left[C W(\theta), C W^{\prime}(\theta)\right] d \theta+\frac{1}{2} \int_{0}^{2 m \pi}\left[C M L C(\theta), C M L C^{\prime}(\theta)\right] d \theta \\
& +\frac{1}{2} \int_{0}^{2 m \pi} \frac{\bar{w}_{\gamma}^{2}}{4 m^{2}}\left[u(\theta), u^{\prime}(\theta)\right] d \theta+\frac{1}{2} \int_{0}^{2 m \pi}\left[C W(\theta), C M L C^{\prime}(\theta)\right] d \theta \\
& +\frac{1}{2} \int_{0}^{2 m \pi} \frac{\bar{w}_{\gamma}}{2 m}\left[C W(\theta), u^{\prime}(\theta)\right] d \theta+\frac{1}{2} \int_{0}^{2 m \pi}\left[C M L C(\theta), C W^{\prime}(\theta)\right] d \theta \\
& +\frac{1}{2} \int_{0}^{2 m \pi} \frac{\bar{w}_{\gamma}}{2 m}\left[C M L C(\theta), u^{\prime}(\theta)\right] d \theta+\frac{1}{2} \int_{0}^{2 m \pi} \frac{\bar{w}_{\gamma}}{2 m}\left[u(\theta), C W^{\prime}(\theta)\right] d \theta \\
& +\frac{1}{2} \int_{0}^{2 m \pi} \frac{\bar{w}_{\gamma}}{2 m}\left[u(\theta), C M L C^{\prime}(\theta)\right] d \theta \\
& =2 \tilde{\mathrm{A}}_{C W(\gamma)}+\tilde{\mathrm{A}}_{C M L C(\gamma)}+\frac{\bar{w}_{\gamma}^{2}}{4 m^{2}} m A_{U}+\frac{1}{2} \int_{0}^{2 m \pi}\left[C M L C(\theta), C W^{\prime}(\theta)\right] d \theta \\
& +\frac{1}{2} \int_{0}^{2 m \pi}\left[C M L C(\theta), C W^{\prime}(\theta)\right] d \theta+\int_{0}^{2 m \pi} \frac{\bar{w}_{\gamma}}{2 m}\left[u(\theta), C W^{\prime}(\theta)\right] d \theta \\
& +\int_{0}^{2 m \pi} \frac{\bar{w}_{\gamma}}{2 m}\left[u(\theta), C M L C^{\prime}(\theta)\right] d \theta \\
& =2 \tilde{\mathrm{A}}_{C W(\gamma)}+\tilde{\mathrm{A}}_{C M L C(\gamma)}+\frac{\bar{w}_{\gamma}^{2}}{4 m^{2}} m \tilde{\mathrm{A}}_{C W}+\frac{\bar{w}_{\gamma}}{2 m(\gamma)} \int_{0}^{2 m \pi}\left[u(\theta), C W^{\prime}(\theta)+C M L C^{\prime}(\theta)\right] d \theta \\
& +\int_{0}^{2 m \pi}\left[C M L C(\theta), C W^{\prime}(\theta)\right] d \theta \\
& (-1)^{(m+1)} L_{v}^{2}(\gamma)=4 m \tilde{\mathrm{A}}_{C M L C(\gamma)}+\frac{L_{v}^{2}(\gamma)}{4 m A_{U}}-\frac{L_{v}^{2}(\gamma)}{4 m A_{U}}\left(1+(-1)^{m}\right) \\
& \\
& \\
&
\end{aligned}
$$


11

\section{Curvas Rosáceas - Discretas}

Considere um plano normado com bola unitária $U=\left\{U_{1}, \ldots, U_{2 n}\right\}$. Conforme a definição (6.2) uma $m$-rosácea discreta

$$
P=\left\{P_{1}, \ldots, P_{2 n}, P_{2 n+1}, \ldots, P_{4 n}, \ldots, P_{2 n(m-1)+1}, \ldots, P_{2 m n}\right\}
$$

é polígono fechado com número de rotações igual a $m$ onde $m$ é um inteiro positivo e para cada $j \in\{1, \ldots, 2 m n\}$ existe um $i \in\{1, \ldots, 2 n\}$ tal que $i \equiv j \bmod (2 n)$ e $P_{j} P_{j+1} \| U_{i} U_{i+1}$. O conjunto das $m$-rosáceas discretas é representado por $C_{U}-m$.

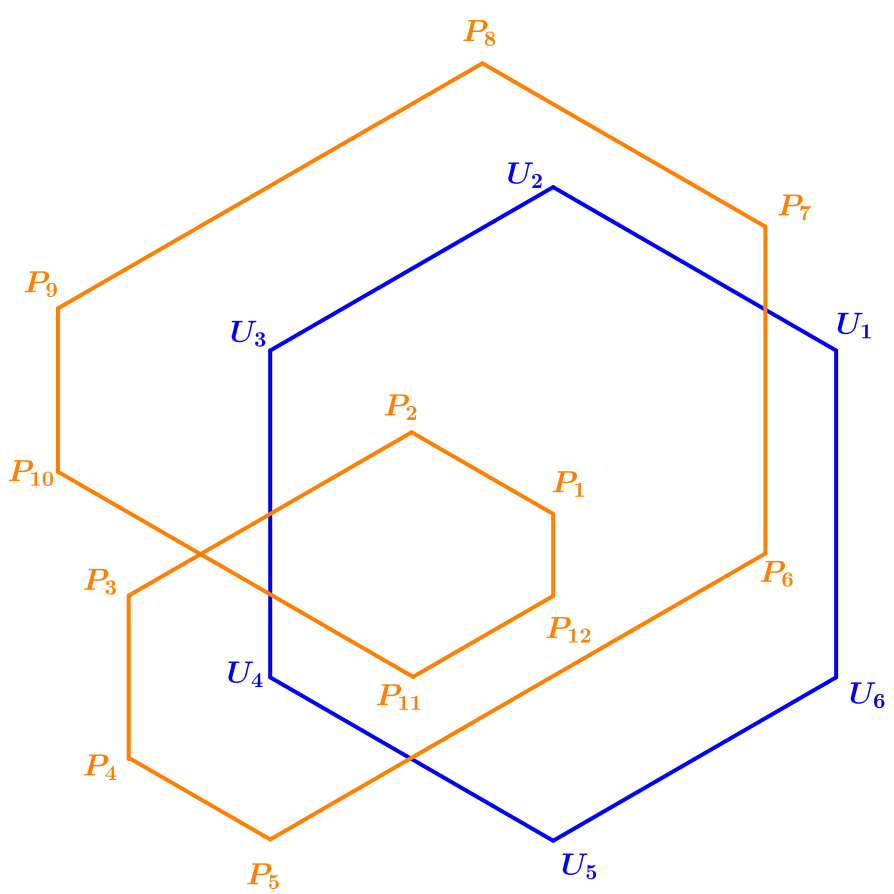

Figura 11.1: Bola unitária $U$ e 2 -rosácea $P$

Para cada $m$-rosácea discreta podemos calcular $\frac{(m+1) m}{2}$ "ramos"de Cáusticas de Wigner. Nós vamos nos deter a apenas um dentre esses "ramos"de Cáusticas de Wigner, pois a partir dele obtemos a igualdade isoperimétrica. Para obter "ramos"de Cáustica de Wigner basta variar $k=1, \ldots, m$ da definição $\left.C W\left(P_{i}\right)=\left(P_{i}+P_{i+k n}\right)\right) / 2$. Vamos nos deter no caso $k=m$. 


\section{1}

\section{Cáustica de Wigner}

Definição 11.1. Seja $P \in C_{U}-m$. Chamamos Evoluta de Área ou Cáustica de Wigner $(C W)$ de $P$ o conjunto

$$
C W(P)=\left\{\left(P_{i}+P_{i+m n}\right) / 2 ; i \in\{1, \ldots, 2 m n\}\right\}
$$

Como $C W\left(P_{i}\right)=C W\left(P_{i+m n}\right)$ temos que $C W(P)$ possui $m n$ vértices. A função suporte de $C W(P)$ é

$$
h_{C W\left(P_{i}\right)}=\left[\left(P_{i}+P_{i+m n}\right) / 2, V_{i}\right]=\frac{1}{2}\left(h_{i}+(-1)^{m} h_{i+m n}\right) .
$$

Onde $h_{i}$ é a função suporte de $P$.

\section{2}

\section{Conjunto de Medida de Largura Constante}

Definição 11.2. Seja $P \in C_{U}-m$. Definimos como Conjunto de Medida de Largura constante de $P$, abreviado por $C M L C(P)$,

$$
C M L C(P)=\left\{\frac{1}{2}\left(P_{i}-P_{i+m n}-\frac{\bar{w}_{P}}{m} U_{i}\right), i \in\{1, \ldots, 2 m n\}\right\}
$$

A função suporte de $C M L C$ é

$$
\begin{aligned}
h_{C M L C\left(P_{i}\right)} & =\frac{1}{2}\left[P_{i}-P_{i+m n}-\frac{\bar{w}_{P}}{m} U_{i}, V_{i}\right] \\
& =\frac{1}{2}\left(h_{i}-(-1)^{m} h_{i+m n}-\frac{\bar{w}_{P}}{m}\right) .
\end{aligned}
$$

11.3

Decomposição do polígono $P \in C_{U}-m$ como soma de Caústica de Wigner, CMLC e bola unitária.

Proposição 11.3. $P \in C_{U}-m, P_{i}=C W\left(P_{i}\right)+C M L C\left(P_{i}\right)+\frac{\bar{w}_{P}}{2 m} U_{i} e$ $h_{P_{i}}=h_{C W\left(P_{i}\right)}+h_{C M L C\left(P_{i}\right)}+\frac{\bar{w}_{P}}{2 m}$. 
Corolário 11.4. $P \in C_{U}-m, P_{i}=C W\left(P_{i}\right)+C M L C\left(P_{i}\right)$ e $h_{P_{i}}=$ $h_{C W\left(P_{i}\right)}+h_{C M L C\left(P_{i}\right)} \Leftrightarrow L_{V}(P)=0$

É simples ver que os resultados acima são consequência do fato que

$$
P_{i}=\frac{P_{i}+P_{i+m n}}{2}+\frac{P_{i}-P_{i+m n}}{2} .
$$

\section{4}

\section{Cúspides CMLC e Cáustica de Wigner}

Teorema 11.5. Considere o polígono $P$ em $C_{U}-m$. Se $m$ é ímpar então o número de cúspides de $C M L C\left(P_{i}\right)$ onde $i \in\{1, \ldots, 2 m n\}$ é múltiplo de quatro. Se $m$ é par e $\bar{w}_{P}=0$ então o número de cúspides de $C M L C\left(P_{i}\right)$ onde $i \in\{1, \ldots, 2 m n\}$ é da forma $2 k, k$ impar.

Demonstração. O número de cúspides de $C M L C(P)$ é igual ao número de trocas de sinal do raio de curvatura em segmentos consecutivos. Sabemos que $r_{C M L C\left(P_{i}\right)}=\left(r_{i}-(-1)^{m} r_{i+m n}-\frac{\bar{w}_{P}}{m}\right) / 2$. Para $m$ ímpar $r_{C M L C\left(P_{i}\right)}=$ $\left(r_{i}+r_{i+m n}-\frac{\bar{w}_{P}}{m}\right) / 2$ o que implica $r_{C M L C\left(P_{i}\right)}=r_{C M L C\left(P_{i+m n}\right)}$. Uma vez que $L_{V}(C M L C(P))=0$ temos

$$
r_{C M L C\left(P_{1}\right)}\left[U_{1}, U_{2}\right]+r_{C M L C\left(P_{2}\right)}\left[U_{2}, U_{3}\right]+\ldots+r_{C M L C\left(P_{m n}\right)}\left[U_{m n}, U_{m n+1}\right]=0
$$

$\mathrm{e}$

$r_{C M L C\left(P_{m n+1}\right)}\left[U_{m n+1}, U_{m n+2}\right]+r_{C M L C\left(P_{m n+2}\right)}\left[U_{m n+2}, U_{m n+3}\right]+\ldots+r_{C M L C\left(P_{2 m n}\right)}\left[U_{2 m n}, U_{2 m n+1}\right]=0$.

Das igualdades anteriores e pelo fato de $\left[U_{i}, U_{i+1}\right]>0, \forall i \in\{1, \ldots, 2 n\}$, concluímos que existe pelo menos um $k \in\{1, \ldots, m n\}$ tal que $r_{C M L C\left(P_{k}\right)}<0$ assim o número de trocas de sinal de $k$ até $k+m n$ deve ser par e pelo menos 2 . O mesmo vale de $k+m n$ até $k+2 m n$. Para $m$ par $r_{C M L C\left(P_{i}\right)}=\left(r_{i}-r_{i+m n}-\frac{\bar{w}_{P}}{m}\right) / 2$. Por hipótese $\bar{w}_{P}=0$ portanto $r_{C M L C\left(P_{i}\right)}=-r_{C M L C\left(P_{i+m n}\right)}$ implica que número de trocas de sinal do raio de curvatura em segmentos consecutivos de 1 até $m n+1$ é ímpar.

Teorema 11.6. Considere o polígono $P$ em $C_{U}-m$. Se $m$ é par então o número de cúspides de $C W\left(P_{i}\right)$ onde $i \in\{1, \ldots, 2 m n\}$ é múltiplo de 4 . Se $m$ 
é impar então o número de cúspides de $C W\left(P_{i}\right)$ onde $i \in\{1, \ldots, 2 m n\}$ é da forma $2 k$, $k$ impar.

Demonstração. Observe que $r_{C W\left(P_{i}\right)}=\frac{r_{i}+(-1)^{m} r_{i+m n}}{2}$. Se $m$ é par então $r_{C W\left(P_{i}\right)}=r_{C W\left(P_{i+m n}\right)}$ então o número de trocas de sinal do raio de curvatura em segmentos consecutivos de 1 até $m n+1$ é par. Se $m$ é ímpar então $r_{C W\left(P_{i}\right)}=-r_{C W\left(P_{i+m n}\right)}$ então o número de trocas de sinal do raio de curvatura em segmentos consecutivos de 1 até $m n+1$ é ímpar.

\section{5}

\section{Exemplo}

Exemplo 11.7. Considere $U=\left\{U_{1}, \ldots, U_{6}\right\}$ bola hexagonal regular e $P \in C_{U}-2$ uma 2-rosácea com raio de curvatura $r=$ $(1 / 2,1,1 / 2,1 / 2,7 / 4,1,1,3 / 2,1 / 2,5 / 4,1 / 2,1 / 4)$. Ver figura 11.1 .

Exemplo 11.8. Considere $U=\left\{U_{1}, U_{2}, U_{3}, U_{4}\right\}$ como bola unitária quadrada, onde $U_{1}=(2,-2), U_{2}=(2,2), U_{3}=(-2,2), U_{4}=(-2,-2)$ e polígono $P$ com raio de curvatura $r=(1 / 4,1,1 / 2,3 / 4,5 / 4,1 / 2,1,3 / 4)$.

Então $L_{V}(P)=\sum_{i=1}^{8} r_{1}\left[U_{i}, U_{i+1}\right]=48 e \bar{w}_{P}=\frac{48}{16}=3$, onde $A(U)=16$. Podemos calcular CW e CMLC.

$C W\left(P_{1}\right)=\left(-\frac{1}{2},-\frac{1}{2}\right)=C W\left(P_{5}\right), C W\left(P_{2}\right)=\left(-\frac{1}{2}, \frac{5}{2}\right)=C W\left(P_{6}\right), C W\left(P_{3}\right)=$ $\left(-\frac{7}{2}, \frac{5}{2}\right)=C W\left(P_{7}\right), C W\left(P_{4}\right)=\left(-\frac{7}{2},-\frac{1}{2}\right)=C W\left(P_{8}\right)$,

$C M L C\left(P_{1}\right)=(-1,2), C M L C\left(P_{2}\right)=(-1,-3), C M L C\left(P_{3}\right)=(1,-3)$, $C M L C\left(P_{4}\right)=(1,1), C M L C\left(P_{5}\right)=(-2,1), C M L C\left(P_{6}\right)=(-2,0)$, $C M L C\left(P_{7}\right)=(2,0), C M L C\left(P_{8}\right)=(2,2)$.

Assim $\tilde{\mathrm{A}}(P)=15, \tilde{\mathrm{A}}(C W(P))=9, \tilde{\mathrm{A}}(C M L C(P))=15, m=2$. Podemos substituir e verificar o teorema (11.7).

$$
\begin{aligned}
& -4 m \tilde{\mathrm{A}}(P) A(U)+8 m \tilde{\mathrm{A}}(C W(P)) A(U)+4 m \tilde{\mathrm{A}}(C M L C(P)) A(U)= \\
& 4 m A(U)(-\tilde{\mathrm{A}}(P)+2 \widetilde{\mathrm{A}}(C W(P))+\widetilde{\mathrm{A}}(C M L C(P))= \\
& 128(-15+18+15)=2304=48^{2}=L_{V}^{2}(P)
\end{aligned}
$$

Ver figura 11.2. 


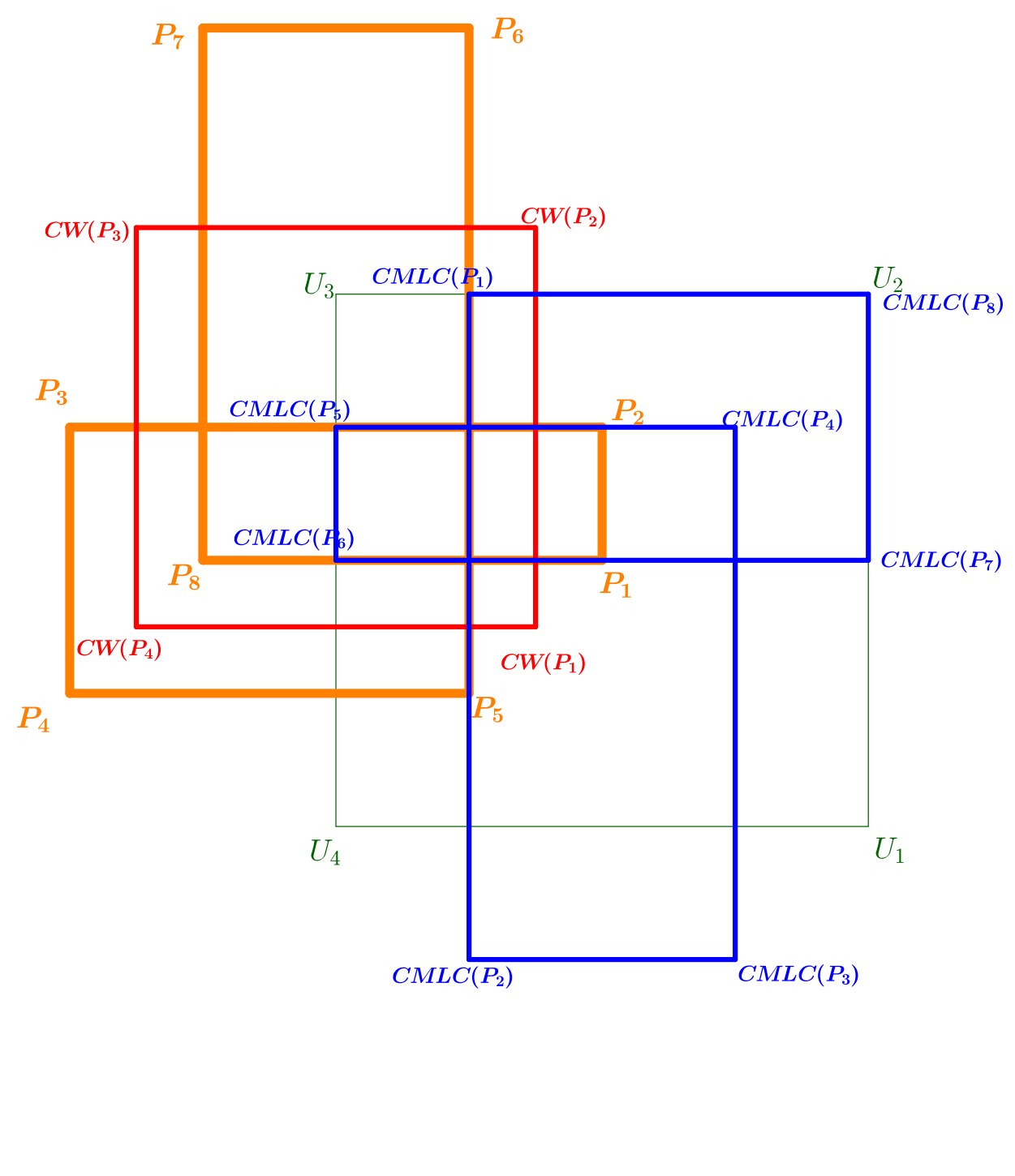

Figura 11.2: Bola U, 2-rosácea P, CW, CMLC

\section{6}

Igualdade Isoperimétrica em Plano Normado para $m$-rosáceas discretas

Teorema 11.9 (Igualdade Isoperimétrica em Plano Normado para polígonos $m$-rosáceas discretas). Seja $P$ uma $m$-rosáceas em $C_{U}-m$ no plano $\left(\mathbb{R}^{2}, U\right)$, onde $U$ é a bola unitária. Então

I) Se m é impar então

$$
L_{V}^{2}(P)=4 m A_{U} \tilde{\mathrm{A}}_{P}-8 m A_{U} \tilde{\mathrm{A}}_{C W(P)}-4 m A_{U} \tilde{\mathrm{A}}_{C M L C(P)} .
$$


II) Se m é par então

$$
L_{V}^{2}(P)=-4 m A_{U} \tilde{\mathrm{A}}_{P}+8 m A_{U} \tilde{\mathrm{A}}_{C W(P)}+4 m A_{U} \widetilde{\mathrm{A}}_{C M L C(P)} .
$$

onde $L_{V}(P)$ indica o v-comprimento com sinal do polígono $P, A_{U}$ a área da bola unitária, $\widetilde{\mathrm{A}}_{P}, \widetilde{\mathrm{A}}_{C W(P)}$ e $\widetilde{\mathrm{A}}_{C M L C(P)}$ indicam respectivamente as áreas com sinal de $P, C W(P)$ e $C M L C(P)$.

\section{Demonstração.}

Pela proposição (11.3) podemos escrever $P_{i}=C W\left(P_{i}\right)+C M L C\left(P_{i}\right)+$ $\frac{\bar{w}_{P}}{2 m} U_{i}$. Utilizando o operador diferença temos $\Delta_{i} P=\Delta_{i} C W(P)+$ $\Delta_{i} C M L C(P)+\frac{\bar{w}_{P}}{2 m} \Delta_{i} U$.

A seguir, temos igualdades importantes para a prova.

$$
\begin{gathered}
\sum_{i=1}^{2 m n}\left[C W\left(P_{i}\right), \Delta_{i} C M L C(P)\right]=-\sum_{i=1}^{2 m n}\left[\Delta_{i} C W(P), C M L C\left(P_{i}\right)\right] \\
\sum_{i=1}^{2 m n} \frac{\bar{w}_{P}}{2 m}\left[C W\left(P_{i}\right), \Delta_{i} U\right]=\sum_{i=1}^{2 m n} \frac{\bar{w}_{P}}{2 m}\left[U_{i}, \Delta_{i} C W(P)\right] \\
\sum_{i=1}^{2 m n} \frac{\bar{w}_{P}}{2 m}\left[C M L C\left(P_{i}\right), \Delta_{i} U\right]=\sum_{i=1}^{2 m n} \frac{\bar{w}_{P}}{2 m}\left[U_{i}, \Delta_{i} C M L C(P)\right] \\
\sum_{i=1}^{2 m n}\left[C M L C\left(P_{i}\right), \Delta_{i} C W(P)\right]=-\frac{L_{V}^{2}(P)}{4 m A_{U}}\left(1+(-1)^{m}\right) \\
\sum_{i=1}^{2 m n}\left[U_{i}, \Delta_{i} C W(P)+\Delta_{i} C M L C(P)\right]=0
\end{gathered}
$$

Vamos calcular a área do polígono P utilizando a proposição (11.3) e as igualdades (11.6), (11.7) e (11.8), (11.9), (11.10) 


$$
\begin{aligned}
& \widetilde{\mathrm{A}}(P)= \\
& =\frac{1}{2} \sum_{i=1}^{2 m n}\left[P_{i}, \Delta_{i} P\right] \\
& =\frac{1}{2} \sum_{i=1}^{2 m n}\left[C W\left(P_{i}\right)+C M L C\left(P_{i}\right)+\frac{\bar{w}_{P}}{2 m} U_{i}, \Delta_{i} C W(P)+\Delta_{i} C M L C(P)+\frac{\bar{w}_{P}}{2 m} \Delta_{i} U\right] \\
& =\frac{1}{2} \sum_{i=1}^{2 m n}\left[C W\left(P_{i}\right), \Delta_{i} C W(P)\right]+\frac{1}{2} \sum_{i=1}^{2 m n}\left[C W\left(P_{i}\right), \Delta_{i} C M L C(P)\right] \\
& +\frac{1}{2} \sum_{i=1}^{2 m n} \frac{\bar{w}_{P}}{2}\left[C W\left(P_{i}\right), \Delta_{i} U\right]+\frac{1}{2} \sum_{i=1}^{2 m n}\left[C M L C\left(P_{i}\right), \Delta_{i} C W(P)\right] \\
& +\frac{1}{2} \sum_{i=1}^{2 m n}\left[C M L C\left(P_{i}\right), \Delta_{i} C M L C(P)\right]+\frac{1}{2} \sum_{i=1}^{2 m n} \frac{\bar{w}_{P}}{2 m}\left[C M L C\left(P_{i}\right), \Delta_{i} U\right] \\
& +\frac{1}{2} \sum_{i=1}^{2 m n} \frac{\bar{w}_{P}}{2 m}\left[U_{i}, \Delta_{i} C W(P)\right]+\frac{1}{2} \sum_{i=1}^{2 m n} \frac{\bar{w}_{P}}{2 m}\left[U_{i}, \Delta_{i} C M L C(P)\right] \\
& +\frac{1}{2} \sum_{i=1}^{2 m n} \frac{\bar{w}_{P}^{2}}{4 m^{2}}\left[U_{i}, \Delta_{i} U\right] \\
& \Rightarrow \\
& \widetilde{\mathrm{A}}(P)=2 \widetilde{\mathrm{A}}_{C W(P)}+\widetilde{\mathrm{A}}_{C M L C(P)}+\frac{\bar{w}_{P}^{2}}{4 m^{2}} m A(U)+\sum_{i=1}^{2 m n} \frac{\bar{w}_{P}}{2 m}\left[C W\left(P_{i}\right), \Delta_{i} U\right] \\
& +\sum_{i=1}^{2 n} \frac{\bar{w}_{P}}{2 m}\left[C M L C\left(P_{i}\right), \Delta_{i} U\right]+\sum_{i=1}^{2 m n}\left[C M L C\left(P_{i}\right), \Delta_{i} C W(P)\right] \\
& \Rightarrow \tilde{\mathrm{A}}(P)=2 \tilde{\mathrm{A}}_{C W(P)}+\tilde{\mathrm{A}}_{C M L C(P)}+\frac{\bar{w}_{P}^{2}}{4 m^{2}} m A(U)-\frac{L_{V}^{2}(P)}{4 m A_{U}}\left(1+(-1)^{m}\right) \\
& \Rightarrow \tilde{\mathrm{A}}(P)=2 \tilde{\mathrm{A}}_{C W(P)}+\widetilde{\mathrm{A}}_{C M L C(P)}+\frac{L_{V}^{2}}{4 m A(U)}-\frac{L_{V}^{2}(P)}{4 m A(U)}\left(1+(-1)^{m}\right) \\
& \Rightarrow \widetilde{\mathrm{A}}(P)=2 \widetilde{\mathrm{A}}_{C W(P)}+\widetilde{\mathrm{A}}_{C M L C(P)}-(-1)^{m} \frac{L_{V}^{2}(P)}{4 m A_{U}} \\
& \Rightarrow(-1)^{(m+1)} L_{V}^{2}(P)=4 m A_{U} \widetilde{\mathrm{A}}_{P}-8 m A_{U} \widetilde{\mathrm{A}}_{C W(P)}-4 m A_{U} \widetilde{\mathrm{A}}_{C M L C(P)}
\end{aligned}
$$

\section{7}

\section{Trabalhos Futuros}

Pensando em trabalhos futuros temos a seguinte pegunta. Podemos definir $C W$ e $C M L C$ em um plano normado geral, ou seja, com a bola unitária convexa mas não necessariamente suave convexa ou poligonal, de forma a obter uma igualdade isoperimétrica? No caso suave, onde utilizamos bolas unitárias suaves, vale a Igualdade isoperimétrica para a classe de curvas "porco espinho". 
No caso discreto, onde utilizamos bolas unitárias poligonais, vale a Igualdade Isoperimétrica para a classe de curvas que são polígonos com lados paralelos a bola poligonal. E no caso geral, qual a classe correta? Outro fato interessante a abordar é se teoremas relativos a outros ramos da Cáustica continuam válidos para bolas não euclidianas. 


\section{Referências bibliográficas}

[1] Dido. Wikipédia, 2019.

[2] CHAVEL, I. Isoperimetric inequalities: differential geometric and analytic perspectives, volume 145. Cambridge University Press, 2001.

[3] CIEŚLAK, W.; MOZGAWA, W. On rosettes and almost rosettes, Geometriae Dedicata, v.24, n.2, p. 221-228, 1987.

[4] CRAIZER, M. Iteration of involutes of constant width curves in the minkowski plane, Beiträge zur Algebra und Geometrie/Contributions to Algebra and Geometry, v.55, n.2, p. 479-496, 2014.

[5] CRAIZER, M.; MARTINI, H. Involutes of polygons of constant width in minkowski planes, arXiv preprint arXiv:1406.3205, 2014.

[6] CRAIZER, M.; TEIXEIRA, R. ; BALESTRO, V. Closed cycloids in a normed plane, Monatshefte für Mathematik, v.185, n.1, p. 43-60, 2018.

[7] CRAIZER, M.; TEIXEIRA, R. ; BALESTRO, V. Discrete cycloids from convex symmetric polygons, Discrete \& Computational Geometry, v.60, n.4, p. 859-884, 2018.

[8] FLANDERS, H. A proof of minkowski's inequality for convex curves, The American Mathematical Monthly, v.75, n.6, p. 581-593, 1968.

[9] FUKUNAGA, T.; TAKAHASHI, M. Evolutes of fronts in the euclidean plane, J. Singul, v.10, p. 92-107, 2014.

[10] GAO, X. A note on the reverse isoperimetric inequality, Results in Mathematics, v.59, n.1-2, p. 83-90, 2011.

[11] GIBLIN, P. Affinely invariant symmetry sets, Geometry and Topology of Caustics (Caustics 06), Banach Center Publications, v.82, p. 71-84, 2008.

[12] GROEMER, H. Geometric applications of Fourier series and spherical harmonics, volume 61. Cambridge University Press, 1996. 
[13] HURWITZ, A. Sur quelques applications géométriques des séries de fourier, Annales scientifiques de l'École Normale Supérieure, v.3e série, 19, p. 357-408, 1902.

[14] LAWLOR, G. A new area-maximization proof for the circle, The Mathematical Intelligencer, v.20, n.1, p. 29-31, 1998.

[15] MIERNOWSKI, A.; MOZGAWA, W. Isoptics of rosettes and rosettes of constant width, Note di Matematica, v.15, n.2, p. 203-213, 1995.

[16] MUSTAFAEV, H. M. Z. On reuleaux triangles in minkowski planes, Contributions to Algebra and Geometry, v.48, n.1, p. 225-235, 2007.

[17] PAN, S.; XU, H. Stability of a reverse isoperimetric inequality, Journal of Mathematical Analysis and Applications, v.350, n.1, p. 348-353, 2009.

[18] PETTY, C. On the geometry of the minkowski plane, Riv. Mat. Univ. Parma, v.6, p. 269-292, 1955.

[19] SCHNEIDER, R. Convex bodies: the Brunn-Minkowski theory. Number 151. Cambridge university press, 2014.

[20] STEINER, J. Sur le maximum et le minimum des figures dans le plan, sur la sphère et dans l'espace en général. premier mémoire., Journal für die reine und angewandte Mathematik, v.24, p. 93-152, 1842.

[21] THOMPSON, A. C.; THOMPSON, A. C. Minkowski geometry, volume 63. Cambridge University Press, 1996.

[22] ZETTL, A. Sturm-liouville theory. Number 121. American Mathematical Soc., 2005.

[23] ZWIERZYŃSKI, M. The constant width measure set, the spherical measure set and isoperimetric equalities for planar ovals, arXiv preprint arXiv:1605.02930, 2016.

[24] ZWIERZYŃSKI, M. The improved isoperimetric inequality and the wigner caustic of planar ovals, Journal of Mathematical Analysis and Applications, v.442, n.2, p. 726-739, 2016.

[25] ZWIERZYŃSKI, M. Isoperimetric equalities for rosettes, arXiv preprint arXiv:1605.08304, 2016. 